RALPH CHRISTENSEN UND KENT D. LERCH

\title{
Performanz
}

\section{Die Kunst, Recht geschehen zu lassen}

Lange ist die Jurisprudenz mit einem einfachen Bild von Recht ausgekommen: Der Richter findet das Recht im Gesetz. Die Gesetze wiederum stehen im Gesetzbuch. Der Richter kann sich also gewiss sein, dass er dort alles findet, was er für sein Urteil braucht. Er muss das Urteil nur noch aus dem Gesetzbuch ableiten. Das Medium des Gesetzbuches garantiert so im Wege der Erkenntnis die Richtigkeit der Entscheidung und damit gleichzeitig ihre Legitimität.

Der Richter und das Gesetzbuch sind aber nicht die einzigen Elemente, die an der Herstellung einer legitimen Entscheidung beteiligt sind. Dazu läuft ein ganzes Verfahren, in dem von anderen Verfahrensbeteiligten Argumente vorgebracht werden. Diese anderen Beteiligten und das Verfahren als Ganzes bleiben allerdings meist außen vor. Das Verfahren ist in der Jurisprudenz nur ein dienendes Instrument. Die Argumentation der Beteiligten und der Verlauf des Verfahrens gelten als defizitäre und nachträgliche Verkörperungen der Gerechtigkeit, die von ihrer Anwendung vollkommen unberührt bleibt. Dabei wird übersehen, dass Recht als soziale Einrichtung aus Kommunikation besteht und dem Verfahren als Ausnutzung von Konfliktperspektiven für die Bildung des Rechts eine offensichtlich zentrale Rolle zukommt.

Man muss daher die herkömmliche Perspektive erweitern. Das Gesetzbuch ist nur der Einstieg in das streitige Verfahren. Es mündet in eine richterliche Begründung, welche die im Verfahren vorgebrachten Argumente verarbeitet. Im Gesetzbuch findet man nicht die Entscheidung des Falles, sondern ein weiteres Medium - die gesprochene Sprache des Verfahrens. Und auch in dieser gesprochenen Sprache sieht man schon den Vorgriff auf ein weiteres Medium: die vom Richter zu schreibende Urteilsbegründung. Da sich das Recht nicht nur eines einzigen Mediums bedient, kann es nicht allein vom Gesetzbuch her be-

\footnotetext{
1 Vgl. dazu Niklas LuhmanN, Konflikt und Recht, in: Ders., Ausdifferenzierung des Rechts. Frankfurt am Main 1999, 92 ff.
} 
griffen werden - sonst wäre alles Wichtige außer Acht gelassen. Recht muss als Medienkonstellation begriffen und analysiert werden.

Die Notwendigkeit $\mathrm{zu}$ einer medientheoretischen Reflexion zeigt sich in der Krise der von der juristischen Methodik vorausgesetzten stabilen und beherrschbaren Sprache, die zwischen Richter und Gesetzbuch vermitteln soll. An ihrer Krise kann man erkennen, dass sie mit dieser Aufgabe überfordert ist.

\section{Die Krise der Rechtssprache}

Die Krise des Gesetzbuchs hat man lange nicht bemerkt, denn das Gleiten seiner Bedeutungen wurde aufgefangen von einem System stabiler Lesegewohnheiten und sozialer Zwänge. Ein Medium, das funktioniert, bleibt aber unsichtbar, denn es liegt im blinden Fleck des Mediengebrauchs. Erst mit dem Wegfall der klassischen Lesegewohnheiten und der sozialen Diversifizierung der Richterschaft wurde die Krise offensichtlich. ${ }^{2}$ Bis dahin war man der Ansicht, das richterliche Urteil könne als gewaltloser Verstandesakt durch lediglich formale Operationen aus der Sprache des Gesetzes abgeleitet werden. Eine solche Sprachkonzeption setzt eine doppelte Einheit des Rechts voraus: Damit die Einheit von Gesetz und Anwendung als kontinuierliche Ableitung der Entscheidung aus dem Gesetz möglich wird, muss das Recht selbst eine Einheit bilden. Garantiert wird diese doppelte Anforderung durch die Theorie einer im Sinnganzen des Buches ruhig gestellten Sprache.

Heute verschwindet die Einheit des Gesetzbuches aber in einer Vielzahl widersprüchlicher Positivierungen, und auch die Anwendung des Gesetzes fügt sich nicht mehr dem Schema der kontinuierlichen Ableitung. Man kann nicht mehr darauf vertrauen, dass das Gesetz spricht. Die Krise des Gesetzbuches ist vor allem die Krise seiner Sprache. Seine Einheit ist bedroht durch die Vielzahl der Lektüren.

\section{Das Schwinden der stabilen Sprache}

Die Krise der Sprache beginnt im 19. Jahrhundert in den Außenbezirken von Philosophie und Literatur, als Sprache plötzlich als eine Lüge empfunden wird. Statt eines selbstverständlichen Mittels des Ausdrucks wird die Sprache immer mehr zu einer Instanz, gegen die Autoren anzuschreiben haben und der sie ihr Werk abringen müssen, scheint die von den Konventionen und Sprachregeln einer repressiven

\footnotetext{
2 Siehe dazu Dieter SimON, Die Unabhängigkeit des Richters. Darmstadt 1975, $21 \mathrm{ff}$.
} 
Gesellschaft geprägte Sprache doch vor allem ein System des Ausschlusses zu sein. ${ }^{3}$ Der Schleier der Gewohnheit hatte verdeckt, was nun mit erschreckender Deutlichkeit hervortrat: dass Sprache auf gesellschaftlicher Vereinbarung beruht und keineswegs auf Erkenntnis und Wahrheit, sondern weit eher auf gesellschaftlichen Konsens abzielt. Dieser Einsicht gibt Nietzsche Ausdruck, nach dem wahrhaft zu sein heißt, "die usuellen Metaphern zu brauchen" und "herdenweise in einem für alle verbindlichen Stile zu lügen". Der gesamte, beeindruckende Begriffsapparat mit seiner pyramidischen Ordnung, seinen Gesetzen, Privilegien, Unterordnungen und Grenzbestimmungen sei auf gleitendem Grund errichtet und seine innere Starrheit eine Abwehrstruktur; aus der menschlichen Praxis hervorgegangen sei die Sprache, ihrem Wahrheitsanspruch zum Trotz, ,durch und durch anthropomorphisch". ${ }^{4}$

Der Glaube an die Wahrhaftigkeit der Sprache wird nun abgelöst von dem Grauen vor ihrer Arbitrarität. Dabei erweist sich die doppelte Bestimmung dieses Begriffs, der sowohl einen Aspekt von Willkür umfasst als auch den scheinbar völlig entgegengesetzten Aspekt einer gesellschaftlichen Determinierung. Einerseits nimmt die Arbitrarität der Sprache jeglichen Anhalt in der Welt, auf die sie sich doch beziehen soll. Sie führt in eine Leere, in den Sturz der Worte der Sprache, ins Bodenlose, erweisen sich diese doch als äußere Zeichen ohne inneren Zusammenhang mit der Wirklichkeit. ${ }^{5}$ Auch wenn die Zeichen aber beliebig sind, so kann man sie doch nicht auf beliebige Art verwenden: der Sprecher muss sich in die willkürliche Gewohnheit der Sprache einordnen, in der er sich dann nur in der vorgeschriebenen Weise ausdrücken kann. Dies ist die andere Seite der Arbitrarität. Sie verweist auf jene dem Diskurs entäußerten Zeichen, die man in Gebrauch nehmen muss, um sich verständlich zu machen. Der gemeinten Bedeutung geben diese aber nicht Ausdruck. Sie muten ihr vielmehr die überkommenen Inhalte zu. Dies wird nun als das Koordinatensystem eines doppelten Schreckens lesbar:

\footnotetext{
${ }^{3}$ Siehe dazu HaRTMUT WINKLER, Docuverse. Zur Medientheorie der Computer. München 1997, 214

${ }^{4}$ FRIEDRICH NiETZSCHE, Über Wahrheit und Lüge im außermoralischen Sinne, in: DERS., Werke. Bd. 5. München 1980, 309 ff., 314, 316 (Erstausgabe 1873).

${ }^{5}$ Siehe auch Hofmannsthals Brief des Lord Chandos aus dem Jahr 1902, in dem die Sprachkrise ihr kanonisiertes Zeugnis gefunden hat, HUGO vON HOFMANNSTHAL, Ein Brief, in: DerS., Gesammelte Werke, Prosa. Bd. II. Frankfurt am Main 1951, 7 ff. Dazu GOTTHART WUNBERG, Der Chandos-Brief, in: DERS., Der friihe Hofmannsthal - Schizophrenie als dichterische Struktur. Stuttgart 1965.
} 
"Die Zeichen haben in der Welt keine zuverlässige Stütze, was sie in einen gefährlichen Schwebezustand versetzt, und sie sind - an die Geschichte gekettet - eben doch keineswegs ,frei'; ihr Vereinbarungscharakter tritt jedem abweichenden Sprechen als ein Widerstand entgegen; und von jeder, Wahrheit' weit entfernt, verweisen sie höhnisch auf die vergangenen Diskurse (die Lügen und Irrtümer der Vergangenheit) zurück."

Nicht nur Literaten und Philosophen leiden aber an der Arbitrarität der Sprache; solches Unbehagen gehört auch zum täglichen Brot des Juristen. Wenn er das Gesetzbuch zur Hand nimmt, um sich für die Entscheidung des Falls einen Begriff vom Recht zu machen, stürzt er in die Probleme des fehlenden Halts. Dabei sind es im Recht nicht nur die allgemeinen Begriffe, wie "Kunst", „Religion", "Meinung" usw., deren Bedeutung im Streit zerfällt; derselbe Vorgang zeigt sich schon bei einer so einfachen Frage, wann es denn „Nacht” sei: Wie dunkel muss es sein, dass wir von Nacht reden? Genügt der Einbruch der Dämmerung, oder wollen wir uns am Sonnenuntergang orientieren? Aber wann ist dieser vollendet und wer entscheidet das?

Der Versuch, mittels des Begriffs zu entscheiden, kollidiert mit der Wendigkeit der Sprache. Was eben noch klare Regel war, kann sich beim nächsten Argument schon wieder als fragwürdig erweisen, denn die Formulierung einer Regel bringt immer die Möglichkeit einer $\mathrm{Ne}$ gation mit sich. ${ }^{7}$ Die Entscheidung über Korrektheit und Konformität von Äußerungen kann nicht durch Sprache vorgegeben sein, nicht einmal die Entscheidung darüber, ob sich die Verwendung eines Ausdrucks noch im Rahmen des Üblichen bewegt - und erst recht nicht vermag es die Regel eindeutig und unwiderruflich vorzuzeichnen, was in jedem Einzelfall gilt. ${ }^{8}$ Der sichere Rechtsbegriff stürzt damit in die offene Frage seiner Bedeutung.

Daran zerbricht der Traum der Juristen, die Welt des Rechts mittels der Begriffe zu beherrschen. In ihrer Arbitrarität ist Sprache systemisch gezeichnet von der Paradoxie einer stets an sich selbst zerfallenden Stabilität. Auch die Rechtssprache ist von einem solchen Wechselspiel von Regel und Ausnahme durchzogen - nur dass die juristische Sicht der Sprache dies leugnet. Sprache gilt im Regelfall als bestimmt und dient als sicheres Instrument juristischer Auslegung. Ausgangspunkt ist dabei ein Wunsch: Als Regelfall gilt der in seiner Bedeutung klare und in seinem Umfang bestimmte Begriff, der ohne die Notwendigkeit einer eigenständigen Wertung für die Subsumtion bereitsteht. Die Ein-

\footnotetext{
6 WINKLER, Docuverse (Fn. 3), 214.

7 Vgl. HANS JÜRGEN HERINGER, Praktische Semantik. Stuttgart 1974, 26.

${ }^{8}$ Siehe das von SAUL A. KRIPKE, Wittgenstein über Regeln und Privatsprache. Eine elementare Darstellung. Frankfurt am Main 1987, insgesamt entwickelte skeptische Argument.
} 
teilungen der Begriffslehre arbeiten sich indes an dem Trauma $a b$, in das diese Idee juristische Praxis stürzt. ${ }^{9}$ Die Art und Weise, in der ein jeweils fraglicher Begriff von dieser Regel abweicht, bestimmt seine Einordnung in der juristischen Begriffslehre: der „unbestimmte Rechtsbegriff" wird meist dadurch definiert, dass Zweifel über seine Anwendung bestehen;10 der "normative Begriff" bedarf einer Wertung, bevor er im Einzelfall angewendet werden kann;"11 der "Ermessensbegriff" geht über die Unbestimmtheit und Wertbezogenheit noch dadurch hinaus, dass er eine persönliche Einstellung des Rechtsanwenders ins Spiel bringt und gerade erst dadurch eine der Einzelfallgerechtigkeit entsprechende Anwendung ermöglicht. ${ }^{12}$

\section{Der Unifizierungswunsch der Juristen}

Das Wuchern der Ausnahmen und mehr noch das in ihrer Systematisierung liegende Eingeständnis, dass sie eher die Regel sind, perforieren unvermeidlich die juristische Idee vom Normalfall sprachlichen Funktionierens. Den Zerfall dieser Idee in der Praxis haben die Juristen aber nicht einfach hingenommen. Gegen das Verstummen, das ihnen ohnehin durch das Gebot zur Entscheidung verwehrt ist, haben sie die Flucht nach vorn in die Theorie angetreten, um sich aller Arbitrarität zum Trotz dennoch eines stabilen Zentrums von Sprache zu versichern. Exemplarisch für den juristischen Wunsch nach einem den Wechselfällen der Sprachpraxis entzogenen Recht sind die methodischen Ausführungen, die das Bundesverfassungsgericht anlässlich der "Soraya"Entscheidung macht:

„Der Richter ist nach dem Grundgesetz nicht darauf verwiesen, gesetzgeberische Weisungen in den Grenzen des möglichen Wortsinns auf den Einzelfall anzuwenden. Eine solche Auffassung würde die grundsätzliche Lückenlosigkeit der positiven staatlichen Rechtsordnung voraussetzen, einen Zustand, der als prinzipielles Postulat der Rechtssicherheit vertretbar, aber praktisch unerreichbar ist. Richterliche Tätigkeit besteht nicht nur im Erkennen und Aussprechen von Entscheidungen des Gesetzgebers. Die Aufgabe der Rechtsprechung kann es insbesondere erfordern, Wertvorstellungen, die der verfassungsmäßigen Rechtsordnung immanent, aber in den Texten der geschriebenen Gesetze nicht oder nur unvollkommen zum Ausdruck ge-

\footnotetext{
${ }^{9}$ Siehe etwa die verschiedenen Aspekte zur Einteilung der Rechtsbegriffe, die ROLF WANK, Die juristische Begriffsbildung. München 1985, 6 ff., benennt.

${ }^{10}$ Vgl. die Nachweise bei KARL ENGISCH, Einfiihrung in das juristische Denken. 8. Aufl., Stuttgart 1983, 257 ff., Fn. 118b, 118c, 119, sowie JAN CATTEPOEL, Der unbestimmte Rechtsbegriff als Problem der Rechtssprache, in: Rechtstheorie 1979, $231 \mathrm{ff}$.

${ }^{11}$ Siehe ENGISCH, Einführung in das juristische Denken (Fn. 10), 259, Fn. 120.

12 Vgl. ebd., 260 ff., Fn. 123 m. w. N.
} 
langt sind, in einem Akt der bewertenden Erkenntnis, dem auch willenhafte Elemente nicht fehlen, ans Licht zu bringen und in Entscheidungen zu realisieren. Der Richter muss sich dabei von Willkür freihalten; seine Entscheidung muss auf rationaler Argumentation beruhen. Es muss einsichtig gemacht werden können, dass das geschriebene Gesetz seine Funktion, ein Rechtsproblem gerecht zu lösen, nicht erfüllt. Die richterliche Entscheidung schließt dann diese Lücke nach den Maßstäben der praktischen Vernunft und den ,fundierten allgemeinen Gerechtigkeitsvorstellungen der Gemeinschaft'."13

Diese Äußerungen zeigen jene Grundbewegung, welche die juristische Zuflucht zur Idee der Gerechtigkeit als stabilisierendem Zentrum ausmacht. Die Erwägungen des Gerichts beginnen beim Verfassungsrecht und münden in die Gerechtigkeitsvorstellungen der Gemeinschaft. Ausgangspunkt ist, dass der Richter nicht darauf verwiesen sei, gesetzgeberische Weisungen in den Grenzen des möglichen Wortlauts auf den Einzelfall anzuwenden. Damit wird zunächst eingestanden, dass sich eine für den Fall ausschlaggebende Bedeutung nicht unmittelbar aus dem Gesetzesbegriff gewinnen lässt. Der Jurist kann also gar nicht verpflichtet sein, dem Gesetzesbegriff unmittelbar seine Bedeutung als Recht zu entnehmen. Aber jetzt wird er an eine zweite Rechtsquelle weitergereicht. Die fehlende Bedeutung wird zur Lücke. Wenn das Gesetz keine vorgegebene Bedeutung hat, so müssen andere Maßstäbe in die Bresche springen. An erster Stelle sind dies die Wertungen, zu denen sich der Jurist durch seinen Fachverstand anleiten lässt. Damit er dabei aber nicht vom rechten Wege abirrt, hat er sich an einem höheren Ganzen zu orientieren: den Gerechtigkeitsvorstellungen der Gemeinschaft.

Die Stationen vom Gesetz über die Lücke und der Wertung zu ihrer Füllung bis hin zur Berufung auf die Idee der Gerechtigkeit markieren exemplarisch die Flucht vor dem Streit der Argumente in die sicher geglaubte Sinnmitte des Rechts. Konzentrisch zu dieser Mitte hin geführt werden die von der Sprache ausgehenden Irritationen durch Delegation an eine Objektivität ruhig gestellt: Die Rechtsidee soll als auBertextuelles Organisationszentrum die Semantisierungen des Normtextes stabilisieren. ${ }^{14}$ Der Weg dahin führt vom Gesetzesbegriff über den Typus oder Gattungsbegriff zu den Rechtsprinzipien, die wiederum eine solche Interpretation anleiten und die Erkenntnis von Recht frei von subjektiver Willkür halten sollen. Auf diese Weise kann jener „Kreislauf zwischen Problementdeckung, Prinzipienbildung und Systemverfestigung" in Gang gesetzt und gehalten werden, der immer

\footnotetext{
${ }^{13}$ BVerfGE 34, 269 ff., 293.

14 Karl LarenZ, Richtiges Recht. München 1979, 33 ff.
} 
wieder den "Geist" des Rechts entdeckt. ${ }^{15}$ Die Sinneinheit der Rechtsordnung als perspektivische Zusammenfügung isolierter Texte unter dem Gedanken der Gerechtigkeit soll so Bedingung wie Ziel der juristischen Arbeit an Recht sein. Das richtige Verstehen einzelner juristischer Texte bedarf eines Vorgriffs auf den Sinnzusammenhang der Rechtsordnung, und dieser garantiert in der Bewegung eines hermeneutischen Einkreisens die Stabilität der Einzelbedeutungen.

Diese juristische Sprachkonzeption verleiht dem nach Sinn suchenden Juristen festen Halt. Mit Hilfe der Begriffe kann er den Rechtsstoff beherrschen und allein aus der Sprache heraus über die Richtigkeit und Angemessenheit der diversen Verwendungen eines Begriffs befinden. Dies setzt allerdings voraus, dass es ein Sprachgesetzbuch gibt, dem die unumstößlichen Regeln richtigen Sprechens ohne weitere Interpretation zu entnehmen sind. Neuerdings nimmt es die Gestalt eines Gesetzbuches diskursiv praktischer Vernunft an. ${ }^{16}$ Danach soll der Geltungsanspruch jenes normativen Sprechaktes, den die Entscheidung von Recht anhand der Normtexte darstellt, durch Herbeiführen eines Konsenses eingelöst werden. Dieser springt in die Bresche, wenn sich die semantische Regel in Vagheit verflüchtigt. Die geforderte Übereinstimmung darf aber keine zufällige sein. Sie hat begründet zu sein, um nicht wieder in die Kontingenz der Arbitraritäten zurückzufallen. Das heißt, der Konsens ist daraufhin zu überprüfen, ob er auch in einer idealen Sprechsituation Bestand hätte, die sich nach bestimmten Regeln beschreiben lässt. Der Prozess der Rechtserzeugung wird so an eine normative Argumentationstheorie gekettet.

An die Stelle des positiv-rechtlichen Gesetzbuches tritt so das ideale Gesetzbuch der praktischen Vernunft. Auch wenn nicht mehr von Rechtserkenntnis, sondern von Rechtserzeugung gesprochen wird, soll diese doch an "anthropologisch tiefsitzende Regeln"17 gebunden sein. Diese sollen jeder Interpretation eine sichere Grundlage verschaffen, indem ein "immer schon" vorausgesetzter universalpragmatischer Code

\footnotetext{
15 Vgl. Karl LarenZ, Methodenlehre der Rechtswissenschaft. 3. Aufl., Berlin 1983, 161. Kritisch zur Typuslehre FRIEDRICH MULLER/RALPH CHRISTENSEN, Juristische Methodik. 9. Aufl., Berlin 2004, Rn. $230 \mathrm{f}$.

16 So bei ROBERT ALEXY, Theorie der juristischen Argumentation. Die Theorie des rationalen Diskurses als Theorie der juristischen Begrïndung. 2. Aufl., Frankfurt am Main 1991; dazu RALPH CHRISTENSEN, Gesetzesbindung oder Bindung an das Gesetzbuch der praktischen Vernunft - Eine skeptische Widerrede zur Vorstellung des sprechenden Textes, in: Rudolf MELlinghoff/HANS-HEINRICH TRUTE (Hrsg.), Die Leistungsfahigkeit des Rechts. Methodik, Gentechnologie, Internationales Verwaltungsrecht. Heidelberg 1988, 95 ff., $104 \mathrm{ff}$.

17 JÜRGEN HABERMAS, Vorbereitende Bemerkungen zu einer Theorie der kommunikativen Kompetenz, in: JÜRGEN HABERMAS/NIKLAS LUHMANN, Theorie der Gesellschaft oder Sozialtechnologie. Frankfurt am Main 1971, 101 ff., 102.
} 
die Begründungsdynamik der richterlichen Rechtserzeugung überwacht. Die Idee eines der Rechtserkenntnis vorgegebenen idealen Gesetzbuches wird damit nicht aufgegeben, sondern nur in die sprachliche Begründungsdynamik zurückgenommen. ${ }^{18}$ Die Sprache bleibt damit feste Burg der Rechtserkenntnis.

Nach wie vor beschreiben die Juristen also Sprache nach dem Muster einer frühzeitlichen agrarischen Gemeinschaft: Bei allen Sprachteilnehmern wird dasselbe Regelwissen vorgehalten. Sollte es doch einmal zu Divergenzen kommen, kann man die Ältesten nach den Prinzipien fragen, und zur Not erhebt der Priester die Stimme der Gerechtigkeit. Die Plausibilität, die einer solchen Geschichte immer noch zukommt, ist nur erklärbar mit der Stärke des Unifizierungswunsches, der dem Differenzierungsdruck der Praxis um jeden Preis entkommen will.

\section{Der Differenzierungsdruck der Praxis}

Jede Verdrängung rächt sich durch ihre Wiederkehr: das gilt auch für das Trauma der Arbitrarität. Um vom Höchsten zu künden, muss der Priester seine Stimme erheben. Orakel haben es aber nun einmal an sich, alles andere als deutlich zu sein. Eher provozieren sie eine Vielfalt der Deutungen, von denen der ganze Berufsstand ihrer Künder lebt. Dies zeigt sich auch in den Ausführungen des Bundesverfassungsgerichts, wenn es von den Maßstäben der praktischen Vernunft und den Gerechtigkeitsvorstellungen der Gemeinschaft im Plural spricht. Weder die praktische Vernunft noch die Gerechtigkeitsvorstellungen sind in einer pluralistischen Gesellschaft hinreichend homogen, um zu garantieren, dass sich ihre Bezeichnungen gegen einen einzigen Sinn austauschen lassen. Wenn sich aber auch diese Größen im Sprechen verändern, ihre ruhige Identität gegen eine Vielheit von Bedeutungserklärungen austauschen, dann gleitet das ganze scheinbar feste und geschlossene System in die Schwerelosigkeit.

Nicht anders ergeht es dem Versuch, das Gesetzbuch als Sinneinheit $\mathrm{zu}$ restituieren. Einerseits muss die Rechtsidee als stabilisierendes Zentrum außerhalb des juristischen Diskurses stehen, um ihn zu kontrollieren, andererseits muss sie genau für diesen Zweck inhaltlich bestimmt werden und fällt so mitten in den Diskurs, den sie doch kontrollieren soll. Jedes Aussprechen und Bestimmen der Gerechtigkeit substituiert die reine Bedeutung durch eine Kette von Zeichen, so dass das Zen-

\footnotetext{
${ }^{18}$ Dazu auch AlEXANDER SOMEK, Unbestimmtheit: Habermas und die Critical Legal Studies, in: Deutsche Zeitschrift für Philosophie 41 (1993), 62 ff.; sowie DIETRICH BUSSE, Juristische Semantik. Berlin 1993, 172 ff.
} 
trum nacheinander verschiedene Namen und Formen erhält. Der vorgebliche Mittelpunkt wird damit genau dem Spiel der Ersetzung unterworfen, das er doch als reiner und mit sich selbst identischer Punkt kontrollieren sollte. Auch dem Versuch, die ideale Sprechsituation in Regeln zu fassen, geht es nicht anders. Mit ihrer Fixierung stellt sich die darin formulierte universelle Vernunft als partikulär heraus.

Die Gemeinsamkeit des Scheiterns aller Versuche, Einkehr zu nehmen in das Paradies einer in sich ruhenden Bedeutung von Sprache, kommt nicht von ungefähr. Sie weist auf ein strukturelles Problem hin. Immer dann, wenn ein transzendentales Signifikat in Anspruch genommen werden soll, ist es genau dadurch keines mehr. ${ }^{19}$ Es bedarf zumindest des erneuten Sündenfalls und eines Verweises darauf. Mit diesem aber geht alle Transzendenz verloren, da es so schon wieder Signifikant ist und als solcher aller Willkür seines Gebrauchs ausgesetzt wird. Der Kokon des Zeichens, der das Signifikat sicher bergen sollte, platzt und entlässt sogleich durch seinen Gebrauch wiederum die entgrenzte Ökonomie der Semantisierungen.

Das ideale Gesetzbuch wird deswegen nur in Sonntagsreden beansprucht. Dem tatsächlichen Differenzierungsdruck der Praxis kann es keinen Widerstand entgegensetzen. Die Berufung auf die Idee der Gerechtigkeit oder die ideale Sprechsituation kann nur als rhetorische Fassade dienen, hinter der sich die faktische Vermehrung und Ausdifferenzierung der Rechtspraxis verbirgt. Andererseits hat aber die Praxis das legitime Bedürfnis, die immer größere Flut von Rechtsinformationen übersichtlich und zugänglich zu machen. Dieses Strukturierungsproblem wird durch die Rhetorik des idealen Gesetzbuches nicht gelöst, sondern erfordert eine Beobachtung der Vervielfältigung des Gesetzes in der Schrift.

\section{Die Ausdifferenzierung des Wissens}

Die Suche nach einer Sinnmitte des Rechts droht nicht allein an der Arbitrarität der Sprache zu scheitern, sondern auch an der Explosion des Wissens. Die Einheit des Wissens und die Stabilität von Regeln sind gefährdet durch die Vielzahl verfügbarer Informationen. Niemand

\footnotetext{
${ }^{19}$ Der Begriff des transzendentalen Signifikats als erster und letzter Quelle des Zeichens geht zurück auf JACQUES DERRIDA, Grammatologie. Frankfurt am Main 1972, 38; er ist bewusst paradox formuliert, denn als transzendental ist die Bedeutung dem sie Bezeichnenden vorausgedacht, das sie erst ermöglicht, als Signifikat trägt sie aber schon alle Bestimmungen der nachträglichen Bezeichnungen an sich; siehe dazu MICHAEL WETZEL, Die Enden des Buches oder die Wiederkehr der Schrift. Weinheim 1991, $14 \mathrm{f}$
} 
kann mehr alles wissen oder wenigstens den Forschungsstand der eigenen Disziplin auch nur annähernd überschauen:

"Es gibt einen wachsenden Berg von Forschungen. Aber gleichzeitig wird zunehmend klar, dass wir uns in einer immer stärkeren Spezialisierung festfahren. Der Forschende ist überwältigt durch die Ergebnisse und Schlussfolgerungen tausender anderer Arbeitender - Schlussfolgerungen, die aufzufassen er keine Zeit findet, geschweige denn sie zu erinnern, wie sie erscheinen. Dennoch wird die Spezialisierung zunehmend wichtig für den Fortschritt, und die Bemühung, zwischen den Disziplinen Brücken zu schlagen, ist entsprechend oberflächlich. Im professionellen Bereich sind unsere Methoden der Übermittlung und Durchsicht von Forschungsergebnissen Generationen alt und den gegenwärtigen Aufgaben in keiner Weise angemessen. ${ }^{20}$

Mit dieser ebenso knappen wie kritischen Analyse wurde 1945 erstmals zur Sprache gebracht, was sich inzwischen zu einem unübersehbaren Problem entwickelt hat: die schnell voranschreitende Arbeitsteilung führt dazu, dass relevante Informationen in einer immer größeren Zahl produziert werden, gleichzeitig aber der einzelne Arbeitende, der diese Informationen eigentlich zur Kenntnis nehmen müsste, von ihrer Fülle und der Geschwindigkeit ihres Erscheinens vollständig überfordert ist.

Grundlegenden Einfluss auf diese Entwicklung hatte dabei die Art und Weise, in der ausdifferenzierte Gesellschaften gemeinsame Wissensbestände produzieren. Während in den frühen agrarischen Gemeinschaften die Individuen einen Großteils ihres Erfahrungsraums teilen, ihre Wissensbestände in nur geringem Maß ausdifferenzieren und ein enges Netz allseitiger Kommunikation Differenzen der Wissensbestände immer wieder ausgleicht, bilden die auf sie folgenden $\mathrm{Ge}$ sellschaften eine arbeitsteilige und damit hoch selektive Grundstruktur aus. ${ }^{21}$ Die Wissensbestände der Individuen treten weit auseinander, so dass gute Voraussetzungen für eine erfolgreiche Kommunikation aufgrund geteilter Erfahrungen nur noch in ausdifferenzierten Subsystemen gegeben sind. ${ }^{22}$ Erst die gesellschaftliche Arbeitsteilung weist den Individuen unterschiedliche Positionen im gesellschaftlichen Netz und damit unterschiedliche Wissensbestände zu. Sie bildet die Grundlage der gesellschaftlichen Struktur und setzt gleichzeitig die Notwendigkeit für einen neuen Typus von Kommunikation: kommuniziert wird

20 Vannevar Bush, As We May Think, in: The Atlantic Monthly 1945, 101 ff. Deutsche Fassung in Auszügen mit Kommentar von Hartmut Winkler in: FormDiskurs 2 (1997), 136 ff. Online: http://wwwcs.upb.de/ winkler/bush_d.pdf

${ }^{21}$ Grundlegend dazu PETER M. HEJ, Wie Gesellschaften Erfahrungen machen oder was Gesellschaftstheorie zum Verständnis des Gedächtnisproblems beitragen kann, in: SIEGFRIED J. SCHMIDT (Hrsg.), Gedächtnis, Probleme und Perspektiven der interdisziplinären Gedächtnisforschung. Frankfurt am Main 1991, 293 ff.

22 WiNKLER, Docuverse (Fn. 3), $116 \mathrm{f}$. 
nicht mehr auf der Basis eines geteilten Erfahrungsraumes, sondern um diesen überhaupt zu konstituieren. Kommunikation bekommt die Aufgabe, die arbeitsteilig differenzierten Positionen miteinander in Beziehung zu setzen.

Eine Gemeinschaft, in der alle mit dem täglichen Nahrungserwerb befasst sind und in der ein jeder für das Ganze zu sorgen hat, ist darauf angewiesen, dass die entsprechenden grundlegenden Fertigkeiten bei jedem einzelnen ihrer Mitglieder und damit gesellschaftlich in einem hohen Maße redundant vorgehalten werden. Erzeugt wird diese Redundanz mit den Mitteln einer mündlichen Traditionsbildung und der Einschreibung der handwerklich-praktischen Fähigkeiten in die Körper, während mythische oder religiöse Systeme für die Kohärenz der Weltbilder sorgen und die regionale Differenzierung eine weitgehende Autarkie der regionalen Einheiten zur Grundlage hat. Mit dem Anwachsen der Gemeinschaft aber stößt dieses System schnell an seine Grenzen. Die Gedächtniskapazität der einzelnen Mitglieder ist limitiert, und die Notwendigkeit, dass jeder alles Wissen vorrätig haben muss, blockiert die weiteren Entwicklungsmöglichkeiten. ${ }^{23}$ Will man diese Grenze überschreiten, so muss die Arbeit und das notwendige Wissen aufgeteilt werden. Dies ermöglicht ein sprunghaftes Anwachsen der gesellschaftlichen Komplexität, die Entwicklung immer speziellerer Technologien und eine regionale Spezialisierung der Produktion bis hin zur internationalen Arbeitsteilung. Hinsichtlich der Verarbeitung von Wissen bedeutet dieser Prozess vor allem die Eliminierung von Redundanz: „Einzelne für die Produktion notwendige Wissensbestände müssen nicht mehr hunderttausendfach, sondern nur noch hundertfach redundant vorgehalten werden, was den Rest der Köpfe und der Körper für neue Wissensbestände freimacht ${ }^{\prime \prime} .{ }^{24}$

Der Prozess der Aufteilung von Arbeit am gesellschaftlichen Wissen bringt für den Einzelnen damit erst einmal eine Entlastung mit sich, braucht er doch nun nicht mehr selbst alle Informationen vorrätig zu halten, wirft aber gleichzeitig auch ernste Folgeprobleme auf: So muss der gesellschaftliche Prozess dafür sorgen, dass der Einzelne zu den für ihn relevanten Informationen kommt und die spezialisierten Tätigkeiten zuverlässig aufeinander bezogen bleiben, was elaborierte Mechanismen der innerbetrieblichen Organisation, der gesellschaftlichen Vermittlung durch den Markt und vergleichbare Institutionen, also eine objektive Vergesellschaftung verlangt. Die objektive Vergesellschaftung allein löst das Problem aber nicht, so dass die Teilung der Arbeit eine Unzahl von Kommunikationsprozessen initiiert und notwendig macht.

${ }^{23}$ WINKLER, Docuverse (Fn. 3), 202.

${ }^{24}$ Ebd. 
Dies ist der systematische Ort, den die Medien einnehmen. Kommunikation findet vor allem statt, um die differierenden Wissensbestände miteinander in Beziehung zu setzen. Arbeitsteilung und (Medien-) Kommunikation sind systematisch und komplementär miteinander verbunden. ${ }^{25}$ Kommunikation wird zum direkten Gegenüber der Arbeitsteilung, weil sie vermitteln muss, was die Arbeitsteilung trennt in der Sache, geographisch und funktional. Es besteht damit eine direkte Beziehung zwischen dem Maß der gesellschaftlichen Differenzierung und dem gesellschaftlichen Kommunikationsbedarf.

Dieses System hat die von Hartmut Winkler in der "Theorie der zwei Köpfe" beschriebene Folge, dass der Kopf jedes Einzelnen in der Gesellschaft eine signifikante Spaltung erfährt:

„Statt eines Kopfes, so könnte man sagen, benötigt jeder nun zwei Köpfe einen nach Fachkompetenz differenzierten Arbeits-Kopf, dessen Spezialisierung ihn zumindest der Tendenz nach von allen anderen spezialisierten Köpfen isoliert, und daneben einen ,allgemeinen', mit dem er ,Mensch' und kommunikationsfähig bleibt. Sache des zweiten Kopfes wäre es, diejenigen Wissensbestände vorzuhalten, die nach wie vor redundant bei jedem Gesellschaftsmitglied vorausgesetzt werden müssen. ${ }^{26}$

Das Auseinandertreten der beiden Köpfe führt zu einer erheblichen Spannung, droht die Spezialisierung der Arbeitsköpfe doch in eine Spezialisierung auch der Weltbilder umzuschlagen. ${ }^{27}$ Alles Bemühen um Öffentlichkeit, gesellschaftliche Kohärenz und Kommunikation muss daher eine zentripetale Kraft entwickeln, die in der Lage ist, dem zentrifugalen Druck der gesellschaftlichen Differenzierung die Waage zu halten. Diese Differenzierung kann aber immer nur so weit voranschreiten, wie die Kohärenz der Kommunikationszusammenhänge garantiert werden kann. Die beiden Köpfe finden sich daher auch unterschiedlich in ihr jeweiliges Medium ein.

25 WINKLER, Docuverse (Fn. 3), 203.

${ }^{26}$ HaRTMUT WinKLER, Die Theorie der zwei Köpfe. Versuch, eine der Wunschstrukturen $\mathrm{zu}$ rekonstruieren, auf die das Datenuniversum eine Antwort ist in: Lab. Jahrbuch 1996/97 für Kïnste und Apparate. Köln 1997, 250 ff. Online: http:/ / www.uni-paderborn. de/ winkler/zweikopf.html.

${ }^{27}$ Das Beispiel der bürgerlichen Salons und der bürgerlichen Öffentlichkeit machen deutlich, wie schwer es ist, das Ideal eines zwanglosen Konsenses gegen die sich verselbständigenden Weltbilder aufrechtzuerhalten, vgl. JÜRGEN HABERMAS, Strukturwandel der Öffentlichkeit. Untersuchungen zu einer Kategorie der bürgerlichen Gesellschaft. Darmstadt 1980 . 
Die Medien, die den ersten, den "Arbeitskopf", mit dem versorgen, was er an Informationen zum Arbeiten braucht, sind vor allem historisch zurückliegende Medien, im Wesentlichen Bücher und Praxen, denn ihm kommt es zu, die spezifischen Wissensbestände auch auf gesellschaftlicher Ebene bereitzuhalten und in ihrer beständigen Ausarbeitung und Detaillierung zu pflegen. Der Einzelne wird so von der Notwendigkeit enthoben, alle Wissensbestände selbst vorrätig zu haben, vielmehr kann er sich darauf verlassen, dass das, was er nicht weiß, von anderen gewusst wird..$^{28}$

Der zweite Kopf hat es dagegen mit avancierteren Medien zu tun: ihn zu stärken und mit Weltbildern auszurüsten, die der Gefahr der Zerreißung und der Verselbstständigung möglichst erfolgreich widerstehen, ist die Leistung, die das Kino, der Rundfunk und das Fernsehen einhundert Jahre lang souverän erbracht haben..$^{29}$ Seine Aufgabe ist es, Integration und Kohärenz zu sichern und damit Kommunikabilität zu unterstützen und erhalten. Genau hier entfaltet sich daher immer wieder aufs Neue der Unifizierungswunsch der Individuen, die Hoffnung nämlich auf die Etablierung eines zentrierten Diskurses, der in der Lage ist, gesellschaftliche Totalität zu repräsentieren. ${ }^{30}$

Mit dem Aufkommen der digitalen Medien wiederholt sich eine Konstellation, die schon beim Siegeszug der Schrift zu beobachten war. Trotz aller Verheißungen birgt sie schon ihre künftige Enttäuschung in sich: Auch wenn sie die Zugänglichkeit, Zuverlässigkeit und Sicherung von Wissen zu verbürgen scheint, so vermehrt sich mit der Verbreitung des Geschriebenen und Gespeicherten das Wissen auf eine Weise, vor der die Bemühungen um eine enzyklopädische Wiederversammlung geradezu als Mut der Verzweiflung erscheinen und von vornherein zum Scheitern verurteilt sein müssen. ${ }^{31}$ Es kommt zu einer förmlichen Explosion des Wissens, gegen die von nun an durch die Institutionen und Mechanismen eines übergreifend gesellschaftlichen Austausches permanent angearbeitet werden muss.

28 WINKLER, Die Theorie der zwei Köpfe (Fn.26).

29 Ebd.

${ }^{30} \mathrm{Ebd}$.

${ }^{31}$ Medientheoretisch dazu Frank HaRTMANN, Medienphilosophie. Wien 2000, $72 \mathrm{ff}$;; zu den Enzyklopädien des Rechts nun grundlegend RAINER MARIA KIESOW, Das Alphabet des Rechts. Frankfurt am Main 2004. 


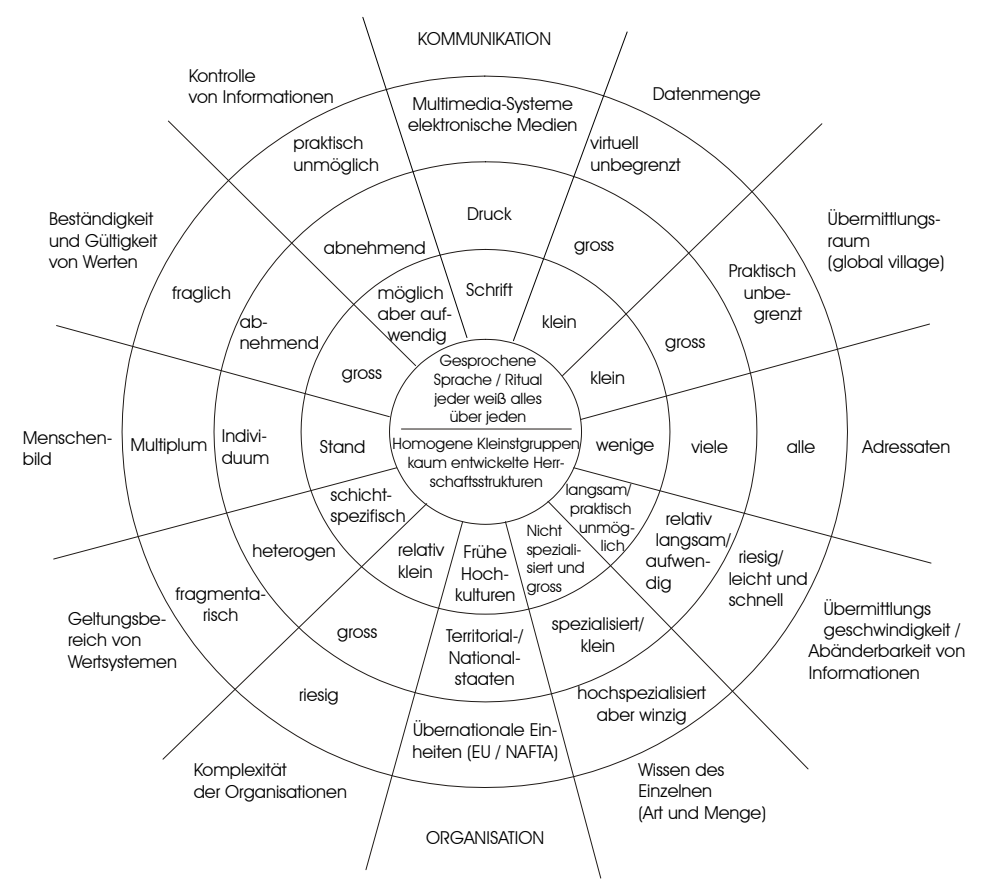

Das vorstehende Diagramm verdeutlicht die Explosion des Wissens. ${ }^{32}$ Den Ausgangspunkt der Entwicklung stellen die archaischen Gemeinschaften dar, in denen Kommunikation und gesellschaftliche Organisation alle Mitglieder der Gruppe in gleicher Weise umfassen. Hauptkommunikationsmittel in diesen Gemeinschaften ist die gesprochene Sprache. Jeder weiß alles über jeden, die Übermittlung ist schnell, und es existiert kein Informationsmonopol. Herrschaftsstrukturen sind kaum entwickelt, Religion, Sitte und Recht bilden eine Einheit. Darum herum liegen drei konzentrische Kreise, die gleichsam die Wellen der Explosion der gesellschaftlichen Organisation wie der in den jeweiligen Systemen verfügbaren Informationen und Kommunikationsmittel darstellen. Die zeitlichen Abstände dieser Explosionswellen werden dabei immer kürzer. Was im Schema gleich groß dargestellt wird, entspricht

\footnotetext{
32 Wir danken Max Baumann für seine freundiche Erlaubnis zum Wiederabdruck des von ihm erstellten Diagramms, erstmals veröffentlicht in: MAX BAUMANN, Recht Sprache - Medien oder Die Notwendigkeit der interdisziplinären Öffnung der Rechtswissenschaft, in: Gesetzgebung heute 1995, 11 ff., 23 f.
} 
Zeiträumen von 5.000 Jahren, 500 Jahren, 5 Jahrzehnten, während wir am äußersten Rand bereits in Kurz- und Kürzestperioden von wenigen Jahren oder zum Teil nur nach Monaten zu rechnen haben.

Mit der allgemeinen Alphabetisierung und Universalisierung des Geschriebenen geht eine unkontrollierbare Differenzierung einher, die den Wissensbestand zunehmend durch Divergenzen aufstört. Die Sicherung schlägt um in Unübersichtlichkeit und die Zugänglichkeit in Desorientierung. In dem Maße, in dem das Geschriebene seine Funktion zu erfüllen vermag, versagt es sich dem Gesellschaftlichen, dem es doch dienen soll. Für die Sprache, die in das Buch gebannt werden sollte und auf die das Recht so unbedingt angewiesen ist, bedeutet dies, dass sie mit dem Voranschreiten der Arbeitsteilung zunehmend belastet wird. Als Instanz der gesellschaftlichen Vermittlung muss sie die auseinander strebenden Fachsprachen und Sprachspiele auf jene Sprache im Singular zurückbeziehen, die die Gesellschaftsmitglieder nach wie vor teilen; die Sprache muss in ihrer inneren Struktur die entstehende Spannung abfangen. ${ }^{33} \mathrm{Um}$ dieses leisten zu können, ist sie allerdings für ihre Überlieferung und Bewahrung genau auf die gleichen Mechanismen wie das Wissen angewiesen und nimmt an deren Scheitern teil. Jeder Buchstabe, durch den sie in Wörterbuch, Grammatik und Konvention festgeschrieben wird, setzt wiederum das Gleiten der sich vervielfältigenden, streuenden und divergierenden Bedeutungen frei.

Die Sprache muss eine explodierende Zahl an Sprechweisen zur Verfügung stellen für alles, was der Fall ist, gleichzeitig aber auch verlässliche Kategorien und Grundbegriffe liefern. Es ist offensichtlich, dass es sich um eine strukturell aporetische Aufgabe handelt: Jede neue Erkenntnis und jede neue Praxis erzwingt eine Differenzierung der Sprache, die dadurch in ein immer ungünstigeres Verhältnis zur beschränkten Kapazität der einzelnen Sprachbenutzer tritt, kann der Bestand an intersubjektiv geteilten Kategorien doch nicht in gleichem Maße zunehmen und sich differenzieren. Die entscheidende Krise tritt daher im Kernbereich der Sprache auf:

„Die zentralen Kategorien, die Abstrakta, werden zunehmend perspektiviert, ihre generalisierende Kraft wird nicht mehr ausreichen, um die differenten Verwendungen zu überbrücken; sie verlieren das Vertrauen und werden in destruktiver Weise schillernd/ambig." ${ }^{\prime 34}$

Mit der Enttäuschung der Sprache aber gerät die Semantik des Gesetzbuches, welche der Rechtserzeugung ihren Halt im Buch garantieren sollte, in die Krise.

33 WINKLER, Docuverse (Fn. 3), 205.

${ }^{34}$ Ebd. 


\section{Die Frage nach dem Medium}

Mit dem Scheitern der Sprache und der Explosion des Wissens schlägt die Stunde der neuen Medien, doch mit den neuen Medien verändern sich auch die Bedingungen des Verstehens. Bisher hat man Textverstehen als Erkenntnis eines im Text vorgegebenen Inhalts begriffen: Die Festigkeit des Textkörpers ist Grundlage für eine ebenso objektive wie sichere Erkenntnis. Es geht um die Feststellung der Logik vorgegebener Formen. In den neuen Medien kommen die Formen dagegen in Fluss: Texte müssen im Netz erst zusammengestellt und wieder auseinander gelegt werden. Die Logik der Formen wird damit ersetzt durch einen Wechsel von Verkörperungs- und Entkörperungspraktiken. An die Stelle der Betrachtung tritt der Vollzug. ${ }^{35}$ Damit verändert sich aber auch die Hermeneutik. Das verfeinerte Verstehen vorgegebener Texte verliert im Textfluss des Netzes seine objektive Basis. Die Verstehenslehre muss damit ihren kontemplativen Charakter ablegen und die Praxis des Kombinierens und Auflösens von Texten reflektieren.

\section{Medientheorie \\ als Frage nach der Verkörperung von Sinn}

Nach wie vor wird im Recht Verstehen aber nicht praktisch, sondern kontemplativ gefasst. Es bezieht sich auf die gedankliche Struktur des Gesetzestextes und ist von der Durchführung eines Verfahrens vollkommen unabhängig. Deswegen wird dem Verfahren in der juristischen Methodenlehre auch keine Beachtung geschenkt. ${ }^{36}$ Es dient nur der didaktischen Vermittlung eines davon unabhängig gefundenen Ergebnisses.

\footnotetext{
${ }^{35}$ Ein Programm muss laufen: „Der Computer, so könnte man sagen, ist das performative Medium schlechthin", HARTMUT WinKLER, Diskursökonomie. Versuch über die innere Ökonomie der Medien. Frankfurt am Main 2004, 226.

${ }^{36}$ Niklas Luhmann hat als Erster das Verfahren in den Mittelpunkt der Analyse gestellt, siehe NiKLAS LUHMANN, Legitimation durch Verfahren. Frankfurt am Main 1983. Allerdings wurden auch in seinen systemtheoretischen Betrachtungen die Programme und die Codierung als unabhängige Größen dem Verfahren vorgeordnet. Erst nach Verarbeitung dekonstruktiver Argumente hat Luhmann konzediert, dass das Recht den Streit der Bürger nicht einfach entscheidet, sondern von diesem Streit in Metamorphosen gezwungen wird, vgl. NIKLAS LUHMANN, Metamorphosen des Staates, in: DERS. Gesellschaftsstruktur und Semantik. Studien zur Wissenssoziologie der modernen Gesellschaft. Band 4. Frankfurt am Main 1999, 101 ff. Siehe dazu auch NIELS WERBER, Vor dem Vertrag. Probleme des Performanzbegriffs aus systemtheoretischer Sicht, in: UWE WIRTH (Hrsg.), Performanz. Zwischen Sprachphilosophie und Kulturwissenschaften. Frankfurt am. Main 2002, 366 ff., vor allem $381 \mathrm{f}$.
} 
Sobald man aber beginnt, das Medium des Verstehens zu thematisieren, wird diese Sichtweise fragwürdig. Genau wie das Buch ist der Computer ein Medium, dessen Einführung die Arbeitsweise der juristischen Praxis erheblich verändert hat. Juristen sitzen nicht mehr vor Büchern, sondern vor dem Bildschirm. Die juristische Methodik hat diese Problematik noch kaum entdeckt, doch man kann nicht annehmen, dass der Kernbereich juristischen Handelns von diesem Wechsel unberührt bleiben wird. Wenn sich die Verkörperungsbedingungen juristischen Sinns so grundlegend verschieben, liegt es nahe, mit der Medientheorie die Hilfe der Disziplin zu bemühen, die solche Vorgänge beobachtet. ${ }^{37}$ Der medial turn der Kulturwissenschaften ist allerdings noch nicht bei den Juristen angelangt. Nach wie vor ist die juristische Hermeneutik fixiert auf das Textverstehen und blendet die Realisierung des Rechts im Verfahren aus.

\section{a) Sprache und Sprachvollzug}

Die Realisierung hat gegenüber der Struktur immer ein Eigengewicht. Dieses Problem ist allerdings nur schrittweise in der Theorie angekommen. Als Leitbegriff hat sich dabei das von Austin etablierte Kunstwort „Performanz" erwiesen. Austin selbst war mit dem Wort nicht glücklich:

"Es ist durchaus verzeihlich, nicht zu wissen, was das Wort performativ bedeutet. Es ist ein neues Wort und ein garstiges Wort, und vielleicht hat es auch keine sonderlich großartige Bedeutung., ${ }^{38}$

Dennoch ist der Begriff der Performanz bedeutsam geworden. Nachdem er zunächst im Gefolge der Rezeption von Chomskys generativer Transformationsgrammatik Karriere gemacht hat, ist er inzwischen in Sprachphilosophie und Kulturwissenschaften allgegenwärtig; entsprechend schillernd sind auch seine Verwendungen. Wissenschaftsgeschichtlich betrachtet hat sich der Begriff der Performanz von einem terminus technicus der Sprechakttheorie zu einem umbrella term der Kul-

\footnotetext{
37 Vgl. zur Einführung WeRnER FaUlSTICH (Hrsg.), Grundwissen Medien. 3. Aufl., München 1998; PETER LUDES, Einfihrung in die Medienwissenschaft. Berlin 1998; DANIELA KlOOCK/AnGela SPAHr, Medientheorien. 2. Aufl., München 2000; JOCHEN HÖRISCH, Der Sinn und die Sinne. Eine Geschichte der Medien. Frankfurt am Main 2001. Zur aktuellen Diskussion vgl. CHRISTOPH ERNST/PETRA GROPP/KARL ANTON SPRENGARD (Hrsg.), Perspektiven interdisziplinärer Medienphilosophie. Bielefeld 2003, dort auch weitere Nachweise in der Einleitung, Fn. 1.

38 JOHN L. AUSTIN, Performative Äußerungen, in: DERS., Gesammelte philosophische Aufsätze. Stuttgart 1986, 305.
} 
turwissenschaften verwandelt..$^{39}$ Es nimmt daher nicht wunder, wenn man schon von einem performative turn spricht:

„Dies ist zum einen Rückgriff auf die Welt als Bühne, als theatrale Praxis, zum anderen auf die Erweiterung einer von den Medien erzwungenen Performance, die damit das Bewusstsein der Inszenierung des Daseins durch die Medien belegt. ${ }^{.40}$

Die wissenschaftliche Verwendung des Begriffs Performanz ist allerdings gekennzeichnet durch eine Ambivalenz zwischen funktionaler und phänomenaler Bestimmung. ${ }^{41}$ Die Spannbreite des ursprünglichen Ausdrucks performance reicht von der Aufführung über die Durchführung, die Leistung bis hin zur Theateraufführung; weitere Verwendungen beziehen sich auf das Fahrverhalten, die Wertentwicklung einer Anlage oder die Geschwindigkeit, mit der Rechner bestimmte Operationen ausführen. Performance meint, so scheint es, den Einsatz von gewissen Fähigkeiten, die Ausführung, den Vollzug. Hier stößt man auf die erwähnte Ambivalenz, denn eine Performance ist nicht nur die Umsetzung von etwas Vorgegebenem, sondern sie fügt mit ihrer Aktion etwas hinzu. Seit in den sechziger Jahren Performance-Künstler auf den Plan traten, weiß man, dass in diesem Sinne Performanz eine Aktion meinen kann, die genau das ist, was sie durchführt.

Führt Performanz also lediglich etwas aus, setzt sie lediglich etwas in die Realität um, das ihr voraus liegt? Ist sie, praktisch gesehen, nur der Einsatz bestimmter Fähigkeiten, die fertig zu Grunde liegen? So kennt man natürlich den Begriff der Performanz aus der Linguistik. Performanz meint demnach die Aktualisierung eines als Kompetenz angelegten Sprachvermögens in den konkreten Äußerungen eines Sprechers, die konkrete Ausführung genereller sprachlicher Strukturen. Hier liegt ein repräsentatives Verständnis von Performanz vor, wie es auch der klassischen Vorstellung in der Jurisprudenz zu Eigen ist: die vorgeordnete Struktur des Rechts bleibt von ihrer Realisierung unberührt. Performanz kann aber auch die Struktur der Realisierung verschieben, so wie ein Stück im Theater nicht einfach aufgeführt, sondern inszeniert wird. Damit tritt an die Stelle des repräsentativen ein präsentatives Verständnis von Performanz. Das repräsentative Verständnis

\footnotetext{
${ }^{39}$ So UWE WIRTH, Der Performanzbegriff im Spannungsfeld von Illokution, Iteration und Indexikalität, in: DERS., Performanz. Zwischen Sprachphilosophie und Kulturwissenschaften. Frankfurt am Main 2002, 9 ff., 10.

${ }^{40}$ GEORG J. LISCHKA, ACT! ON, Aktion und Reaktion - Performance und Interaktion, in: GeOrg J. LischKa/Peter Weibel, ACT!, Handlungsformen in Kunst und Politik. Bern 2004, 7 ff., 20; vgl. dazu auch ALICE LAGAAY/DAVID LAUER, Einleitung - Medientheorien aus philosophischer Sicht, in: DIES. (Hrsg.), Medientheorien. Eine philosophische Einfiihrung. Frankfurt am Main 2004, 7 ff., 13.

${ }^{41}$ WrRTH, Der Performanzbegriff (Fn. 39), 9 ff., 10.
} 
führt zu einer epistemischen Evidenz im Bewusstsein der Beteiligten, welche lediglich aufgedeckt wird. Das präsentative Verständnis will dagegen im Verfahren Evidenz erst herstellen, so dass sich auch etwas Neues ereignen kann. Erst mit diesem Verständnis kann man dem Verfahren einen eigenen Wert zubilligen. Das Verfahren ist für die Entstehung von Recht konstitutiv.

Die Ambivalenz der Verwendung von Performanz beginnt, als Austin Sprache als Handeln entdeckte. Seine Vorlesungen galten eben nicht primär einer "Theorie der Sprechakte", wie es der verunglückte Titel der deutschen Ausgabe weismachen will: "How to do things with words", war die Frage, die ihn bewegte. Das hat die deutsche Ausgabe mit etwas schlechtem Gewissen ihrer Titulierung in Klammern hinzugefügt. An dieser Spannweite des Problems konnte die dekonstruktive Kritik ansetzen, um dem Begriff der Performanz sein pragmatisches Potential zurückzuerstatten, das der Begriff auf seinem Weg von Chomsky über Searle in die Transzendentalpragmatik verloren hatte; dabei wurde die Frage nach den funktionalen Gelingensbedingungen der Sprechakte von der Frage nach ihren phänomenalen Verkörperungsbedingungen abgelöst. ${ }^{42}$

Schon der Begriff Praxis eröffnete die Möglichkeit, Sprechen als Handeln zu begreifen. Diesen Weg hat die Sprachphilosophie im Anschluss an Wittgenstein dann auch eingeschlagen, nachdem dieser den Begriff der Bedeutung in die Frage nach einer Praxis von Sprache aufgelöst hatte: „Was bezeichnen nun die Wörter dieser Sprache? - Was sie bezeichnen, wie soll ich das zeigen, es sei denn in der Art ihres Gebrauchs?" ${ }^{43}$ Allerdings wurde Wittgenstein von seinen Epigonen meist verkürzt auf die Anweisung: „Man kann für eine große Klasse von Fällen der Benützung des Wortes ,Bedeutung' - wenn auch nicht für alle Fälle - dieses Wort so erklären: Die Bedeutung eines Wortes ist sein Gebrauch in der Sprache. ${ }^{44}$

Austin wollte dabei nicht stehen bleiben und profitierte so vom Nutzen ungelöster Probleme. ${ }^{45}$ Er entdeckte die von ihm „explizit performativ" genannten Äußerungen, deren Vollzug an die Verwendung

42 WIRTH, Der Performanzbegriff (Fn. 39), 9 ff., 9 f.

${ }^{43}$ LuDWIG WITTGENSTEIN, Philosophische Untersuchungen. Werkausgabe Bd.1. Frankfurt am Main 1984, § 10.

${ }^{44}$ WitTGENSTEIN, Philosophische Untersuchungen (Fn. 43), $\$ 43$.

${ }^{45}$ Die Verschiebung eines Problems ist manchmal der Lösung überlegen, vgl. dazu DiRK BAECKER, Vom Nutzen ungelöster Probleme, in: DiRK BAECKER/ALEXANDER KLUGE, Vom Nutzen ungelöster Probleme. Berlin 2004, 33 ff. 51: „Daraus resultiert unser Interesse an Struktur und Semantik von so genannten ,Experimenten' und ,Spielräumen', in denen wir Möglichkeiten ausloten können und dies auf eine Art und Weise tun, die die Variationen von morgen heute schon in Reserve hält." 
bestimmter Verben wie "taufen" oder "erklären $z u$ “ gebunden ist: so wird mit bestimmten Äußerungen genau das getan, was sie ausdrücken. Nimmt man, um eines der berühmten Beispiele zu beanspruchen, einen Satz wie "Ich taufe dich auf den Namen Waldo", so liegt seine Bedeutung darin, dass er vollzieht, was er sagt. Dies macht deutlich, dass die "konstativen" Äußerungen nicht alles abdecken und die repräsentativ wahrheitsfähige Verwendung von Sprache nicht der einzige Gegenstand der Betrachtung bleiben kann.

Die entscheidende Frage, was es mit der sprachlichen Bedeutung auf sich hat, ist damit allerdings nicht beantwortet. Vielmehr stellt sie sich für Austin erst einmal neu: Wie ist es möglich, eine Handlung durch das Äußern eines Satzes auszuführen? Die Bedeutung stützt sich auf nichts anderes als die Ausführung. Aber die Möglichkeitsbedingungen müssen sich aufweisen lassen. Dies folgt aus dem Umstand, dass Äußerungen einer bestimmten Form bedürfen und auch schief gehen können. Wenn etwas scheitern kann, muss es bestimmte Bedingungen für das Gelingen geben, an denen sich das Misslingen bemisst.

Mit dieser Grundfigur ist der Konventionalismus der Sprechakttheorie eingeführt. Er unterwirft die Performanz einer übergeordneten und regierenden Typologie. Dafür einstehen sollen „conventional procedures", die die Bedingungen für den gelungenen Vollzug regeln. Vorderhand ist dies auch kaum bestreitbar. Nicht jede Äußerung des Satzes "Hiermit taufe ich dich auf den Namen Waldo" kann als rechtskräftiger Vollzug der Taufe gelten. Es bedarf etwa eines dafür autorisiert eingesetzten Sprechers. Zudem muss der Satz dann mit aller „Ernsthaftigkeit" ausgesprochen werden. Ist er Teil eines Schauspiels, so kann er den Taufakt allenfalls virtuell ausführen. Eine vollgültige Taufe, die den betroffenen Schauspieler zwingen würde, sich fortan als „Waldo" vorzustellen, ist damit jedoch nicht vor sich gegangen. Das Ereignis hat dennoch alle Züge einer Taufe. Das gleiche gilt erst recht für nicht explizite und dennoch performative sprachliche Äußerungen wie etwa "Ich verspreche dir, heute Abend zu kommen“. Diese Klasse von ÄuBerungen bringt neue Komplikationen mit sich. Auf der einen Seite brauchen sie für ihren Vollzug nicht die explizit selbstbezogene Verbalisierung dessen, was sie sein sollen. Eine Äußerung wie "Ja, ja, ich bin

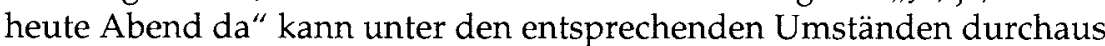
ein Versprechen mit allen Konsequenzen darstellen. Umgekehrt muss nicht alles, was sich Versprechen nennt, ein solches sein. Eine Äußerung wie "Ich verspreche dir, das wird dir noch Leid tun" wird man wohl eher als Drohung oder Warnung verstehen.

Austin zieht daraus Konsequenzen. Er bindet das Gelingen des Sprechakts an Regularien, die er ex negativo aus einer Taxonomie von 
Fehlschlägen gewinnt. Die Bedingungen für den gelungenen Vollzug von Sprechakten sollen unter normalen Umständen seine Geltung garantieren und machen zugleich die Bedeutung der performativen ÄuBerung aus. Der Tatsache, dass man mit einem solchen Akt dann durchaus verschiedenes meinen und bewirken kann, trägt Austin mit der Unterscheidung von illokutionären und perlokutionären Akten Rechnung. ${ }^{46}$ Die Illokution ist der Vollzug einer Handlung, während die Perlokution die Wirkungen auf den Rezipienten betreffen. Diese Wirkungen sind nicht vollständig konventionalisiert. Dabei können sich die Wirkungen sowohl dem Meinen des Sprechers als auch jeder Einbettung in Konventionen entziehen. Die Perlokution ist damit ein Element, was in der Typologie nicht vollständig erfasst werden kann. ${ }^{47}$

Die Abweichung vom Rahmen der Konvention kann allerdings auch im Bereich der Illokution auftreten. Für die klassische Sprechakttheorie ist dies allerdings nur ein Indiz für ein Misslingen. Tatsächlich kann es aber einen Bedeutungsüberschuss kenntlich machen. Das Abweichen von der Regel will etwas bedeuten. So ist bei einer als Versprechen auftretenden Drohung der Sprechakt keineswegs misslungen, genau so wie bei den sogenannten ",indirekten Sprechakten“ wie ironischen ÄuBerungen, ${ }^{48}$ bei denen zwar mit aller ", $\mathrm{Kraft}^{\prime}$ das eine gesagt, mit voller Absicht aber ein anderes getan ist. Der Akt wird in eine andere Form gekleidet. Dahinter kann eine Vielzahl von Gründen stehen, seien es nun Höflichkeit oder Sanktionsscheu. Diese erzeugen den Bedeutungsüberschuss.

Austin kann das Phänomen dieses Bedeutungsüberschusses nicht erfassen und kommt daher zu dem Urteil, die Sprache werde "auf ganz bestimmte, dabei verständliche und durchschaubare Weise unernst gebraucht, und zwar wird der gewöhnliche Gebrauch parasitär ausgenutzt. ${ }^{.49}$ Der Parasit ernährt sich dabei von der Kraft der Konventionen. Diese sind Gelingensbedingungen für jeden Sprechakt.

Der Konventionalismus erfährt noch einmal eine Verschärfung durch die Ausarbeitung der Sprechakttheorie in systematischer Absicht bei Searle. ${ }^{50}$ Während der Begriff der Performanz in den Vorlesungen

\footnotetext{
${ }^{46}$ JOHN L. AUSTIN, Zur Theorie der Sprechakte. Stuttgart 1975, 117.

${ }^{47}$ Der perlokutionäre Akt betrifft die "kürzere oder längere Kette von ,Wirkungen'”, welche der Sprechakt auf einen Rezipienten ausübt, wobei er den konventionalen Rahmen illokutionärer Effekte durchaus sprengen kann, AUSTIN, Zur Theorie der Sprechakte (Fn. 46), 124.

${ }^{48}$ Dazu John R. Searle, Indirect Speech Acts, in: Peter Cole/Jerry Morgan (Hrsg.), Syntax and Semantics 3: Speech Acts. New York 1975, 59 ff.

${ }^{49}$ AUSTIN, Zur Theorie der Sprechakte (Fn. 46), $43 \mathrm{f}$.

${ }^{50}$ Siehe JOHN R. SEARLE, Sprechakte. Ein sprachphilosophischer Essay. Frankfurt am Main 1969.
} 
von Austin durchaus noch offen und tastend bleibt und von diesem eher umkreist als festgeschrieben wird, schließt Searle dafür ausdrücklich affirmativ und kritisch zugleich an dessen einflussreiche linguistische Bestimmung durch Chomsky an. ${ }^{51} \mathrm{Im}$ Rahmen der generativen Transformationsgrammatik sollte Performanz lediglich die jeweils konkret aktuelle Umsetzung von Sprachstrukturen bezeichnen, die als universales Vermögen die Kompetenz eines Sprechers ausmachen. Wesentlich dabei ist das Verhältnis, in das beide damit gesetzt werden. Performanz ist als die Aktualisierung von Sprachstrukturen in konkret situierten Äußerungen immer sekundär, als ein mehr oder weniger gelungener Abklatsch der reinen Formen von Sprache. Die Kompetenz als allgemeines „Kenntnissystem" bestimmt die Form der Sprache. Der Performanz als "aktualem Gebrauch" eignet dagegen immer auch das Moment einer sprachlichen Deformation. Die Sprache als beobachtbares Phänomen ist demnach immer eine Verzerrung der "reinen Sprache “. ${ }^{22}$ Der Akzent liegt hier - ganz im Sinne der klassischen juristischen Auffassung - auf der idealen Struktur. Die Realisierung gilt als zufällig und defizitär.

Austins Beobachtung parasitärer Ausnutzung konventionaler Prozeduren wird hier generalisiert. Die Konventionen werden zum Wesenszug der Äußerungspraxis überhaupt. Genau dieses deduktive Ableitungsverhältnis übernimmt auch Searle für seinen Anspruch auf eine Grammatisierung des Austin'schen Sprechaktkonzepts. Searle geht dabei über Chomsky hinaus, indem er die Ausklammerung von Bedeutungsfragen aus der syntaxorientierten Transformationsgrammatik überwinden will. Er findet die fehlende Bedeutung von Äußerungen in den durch sie vollzogenen Handlungen. In ihrer Form als geltender Akt werden diese durch die dafür "konstitutiven“ Regeln bestimmt. Diese legen in einer minutiösen Faktorenbestimmung die Bedingungen fest, die eine Äußerung erfüllen muss, damit durch sie etwa ein Versprechen abgegeben wurde, wobei Teil dieser Bedingungen dann auch die Verpflichtungen sind, die Sprecher und Hörer kraft des Vollzugs des entsprechenden Sprechakts eingehen. Realisiert sind diese dann durch den „performativen Vollzug“ in der konkreten Sprecheräußerung.

Ganz so wie bei Chomsky etabliert also auch bei Searle die Idealisierung der Kompetenz ein deduktives Ableitungsverhältnis, welches jeden induktiven Rückschluss von der phänomenalen Ebene der Per-

\footnotetext{
51 Siehe NOAM CHOMSKY, Aspekte einer Syntax-Theorie. Frankfurt am Main 1965.

52 WIRTH, Der Performanzbegriff (Fn. 39), 9 ff., 12. Zum Ganzen auch SIBYLLE KRÄMER, Sprache, Sprechakt, Kommunikation. Frankfurt am Main 2001.
} 
formanz auf die Ebene der Kompetenz von vornherein ausschließt. ${ }^{53}$ In der Sprechakttheorie ist dies ein Verhältnis von vorgeordnetem Sinn zu nachgeordnetem Vollzug; sie trifft eine Entscheidung für den abstrakten Typ und vernachlässigt die Realisierung als notwendig defizitär. Wesentlich ist allein, inwieweit die Performanz sich getreu dem konstitutiv Grammatischen der Form verhält. Was der Sprecher mit ihr sonst noch beabsichtigt, ist für die sprachliche Bedeutung von keinerlei Interesse mehr.

Seine Verlängerung ins Transzendentale erfährt dieses Konzept mit der Rezeption der Sprechakttheorie durch die Transzendentalpragmatik. ${ }^{54}$ Searle lässt den endgültigen Status der den Vollzug von Sprechakten anleitenden Regeln offen und sieht in ihnen lediglich das Bedeutung konstituierende Moment. Bei Habermas gewinnen diese Regeln den Stellenwert universeller Strukturen kommunikativen Handelns, aus denen alle Performanz entspringt. Searles Handlungsgrammatik gerät so unter dem Anspruch ihrer diskursethischen Reformulierung zur transzendental kommunikationspolizeilichen Vorkehrung. Für Searle ist sprachliches Verstehen die Einsicht in die Intention des Sprechers. Aber diese Intention ist vollständig durch die semantischen Konventionen des propositionalen Gehalts und auch durch die pragmatischen Konventionen der illokutionären Funktion bestimmt. Diese Dominanz der Konventionen über die Illokution erlaubt für Apel und Habermas die Ableitung ethischer Normen aus universellen Sprachregeln. ${ }^{55}$ Seinen deutlichsten Ausdruck findet dies in der Figur des performativen Widerspruchs. ${ }^{56}$ Der Widerspruch zwischen dem, was ein Text oder ein Sprecher tut, und dem, was er sagt, wird hier nicht wie in der Dekonstruktion zur Entdeckung von Problemen verwendet; diese Probleme werden vielmehr verdeckt, indem der performative Widerspruch das vollkommene Scheitern der Äußerung anzeigt. An die Stelle einer Problemdiskussion tritt der Vorwurf, nicht etwa die pragmatischen Regeln des Gelingens in Frage zu stellen, sondern die universellen Geltungsbedingungen der Kommunikation zu verfehlen.

\footnotetext{
${ }^{53}$ WIRTH, Der Performanzbegriff (Fn. 39), 9 ff., 12.

54 Siehe JURGEN HABERMAS, Theorie kommunikativen Handelns. Frankfurt am Main 1981, und KARL OTTO APEL, Sprechakttheorie und transzendentale Sprachpragmatik zur Frage ethischer Normen, in: DERS., (Hrsg.), Sprachpragmatik und Philosophie. Frankfurt am Main 1976, $10 \mathrm{ff}$.

55 WIRTH, Der Performanzbegriff (Fn. 39), 9 ff., 14 f.

56 Dazu Rudolf Gebauer, Jürgen Habermas und das Prinzip des zu vermeidenden performativen Widerspruchs, in: Allgemeine Zeitschrift fïr Philosophie 1993, 23 ff. Kritisch in Bezug auf juristisches Handeln RALPH CHRISTENSEN, Gesetzesbindung oder Bindung an das Gesetzbuch der praktischen Vernunft (Fn. 16), 95 ff., 104 ff. m. w. N.
} 
Die Überhöhung pragmatischer Regeln zu universellen Strukturen wertet dabei die Praxis noch stärker ab. Die Realisierung kann die Praxis weder in Frage stellen, noch verschieben. Sie ist der Struktur vollkommen untergeordnet und muss sich bei Abweichung den Vorwurf des Defizits gefallen lassen. Der Maßstab liegt immer bei der Struktur und niemals bei der Realisierung. Die Einlinigkeit dieser Betrachtung wird dadurch plausibel, dass die Defizite der Realisierung auffälliger sind als ein allmähliches Verschieben der Struktur durch die Praxis. Defizite springen ins Auge. So wirkt es merkwürdig, wenn ein Sprecher sich anschickt, eine bestimmte Handlung zu vollziehen, in diesem Vollzug aber zugleich für deren Geltung ausschlaggebende Momente dementiert. Gibt jemand ein Versprechen und sagt gleich dazu, er werde sich auf keinen Fall daran halten, so ruft dies Befremden hervor. Die Pointe der Universalpragmatik ist, dass sie nun keineswegs von der Performanz zurückfragt auf die besondere Bedeutung, die die Äußerung genau aus diesem Umstand gewinnt; dies wäre der Ansatz der konversationellen Implikatur von Grice, der solch innere Widersprüchlichkeit zum Anlass nimmt, die betreffende Äußerung unter den "Verdacht" eines besonderen Sinns zu stellen, um damit die Bedeutung der Äußerung aus der „kommunikativen Logik“ ihrer Darbietung zu gewinnen. ${ }^{57}$ Ganz im Gegensatz dazu führt die Universalpragmatik Inkonsistenzen im Vollzug gegen den Sprecher ins Feld. Sie rechtfertigen es, den Sprecher in die Schranken der Universalregeln zu weisen oder aber seinen Akt aufgrund eines Verstoßes gegen diese für ungültig zu erklären.

Die Transzendental- und Universalpragmatik vollendet die Überhöhung des Systematischen zu Lasten der Performanz. Sie lässt nur unproblematische „ideale Gesprächssituationen“ oder "pragmatische Universalien" gelten, unter deren Schirmherrschaft ernsthaft kommuniziert werden kann. ${ }^{58}$ Der sachliche Kern, der in dieser Übersteigerung sichtbar wird, ist der Konventionalismus. Diese Privilegierung der Regel würde die herkömmliche Auffassung in der Rechtstheorie bestätigen, wonach im Prozess die Erkenntnis des Rechts zwar scheitern, dieser Prozess das Recht aber niemals verschieben kann.

\footnotetext{
57 Dazu HeRbERT P. GRICE, Logik und Konversation, in: GEORG MEGGLE (Hrsg.), Handlung, Kommunikation, Bedeutung. Frankfurt am Main 1979, $243 \mathrm{ff}$.

58 MANFRED FRANK, Was ist Neostrukturalismus?, Frankfurt am Main 1983, $513 \mathrm{f}$.
} 


\section{b) Sprachvollzug und Iteration}

Natürlich kann eine Analyse wirklicher Sprache bei der Idealisierung der Regelhaftigkeit nicht stehen bleiben. Die Rückwirkung des Vollzugs auf die Struktur springt zwar nicht in gleicher Weise ins Auge wie das Scheitern eines Sprechakts, aber gleichwohl ist sie für die sprachwissenschaftliche Forschung nicht zu übersehen. Die Kritik am übersteigerten Konventionalismus arbeitet die Eigengesetzlichkeit und die produktive Rolle der Realisierung heraus.

Die innerhalb der analytischen Tradition entwickelte Kritik von Grice und Davidson hat die konventionalistische Privilegierung des Sprechers gegenüber dem Sprachverstehen umgekehrt. ${ }^{59}$ Die dekonstruktivistische Kritik setzt demgegenüber an Austins Behandlung der konventionalen Gelingensbedingungen von Sprechakten an, begreift er doch die Möglichkeit des Scheiterns von konventionellen Prozeduren lediglich als Eventualität und nicht als notwendige Eigenschaft der Sprachverwendung. Austin glaubte, den parasitären Gebrauch der Sprache "mit voller Absicht" aus seiner Untersuchung ausschließen zu können, weil es sich um eine „zusätzliche“ Art des Gebrauchs handele, die über den normalen, konventionalen Gebrauch hinausgehe. ${ }^{60}$ Die Metapher des Parasiten impliziert dabei ein klares Abhängigkeitsverhältnis. Der Parasit lebt nicht nur "auf" der Wirtspflanze, er ist notwendigerweise auf sie angewiesen: ohne sie könnte er nicht überleben, wohingegen die Wirtspflanze sehr gut ohne den Parasit auskommen kann. ${ }^{61}$ Das ist die übliche Vorstellung vom Parasiten: Er stört und man glaubt, ihn ausmerzen zu können. Aber vielleicht sind ja Parasiten die notwendigen Zwischenhändler oder Vermittler jeder Information. ${ }^{62}$ Vielleicht kann man ihn gar nicht ausmerzen, wenn man kommunizieren will, sondern hat lediglich die Wahl, sich um ein nützliches Verhältnis zu bemühen. Im Konventionalismus aber verschwindet der Parasit. Performanz soll lediglich den konventionellen Prozeduren aufsitzen, um von diesen zu zehren. Alles, was sich dem Konzept nicht fügt, ist so, falls es überhaupt noch verstanden werden kann, nur Abweichung; es hat für die Genese und Feststellung von Sinn keinerlei

\footnotetext{
${ }^{59}$ Siehe DONALD DAVIDSON, Kommunikation und Konvention, in: DERS., Wahrheit und Interpretation. Frankfurt am Main 1990, $372 \mathrm{ff}$.

${ }^{60}$ AUSTIN, Zur Theorie der Sprechakte (Fn. 46), 43 f.

${ }^{61}$ WIRTH, Der Performanzbegriff (Fn. 39), 9 ff., 22.

62 Vgl. zu diesem Verdacht MiChel SerRes, Der Parasit. Frankfurt am Main 1981. Dazu auch NikLas LuHMANN, Die Politik der Gesellschaft. Frankfurt am Main 2000, $378 \mathrm{f}$. Grundlegend zur Figur des Parasiten im Recht: Matthias KronenberGer, Der Parasit der Überzeugungsbildung. Diss., Frankfurt 2005 (im Erscheinen).
} 
Bedeutung. ${ }^{63}$ Die herkömmliche Auffassung der Sprechakttheorie geht deshalb davon aus, dass eine trennscharfe Unterscheidung zwischen normalem und parasitärem Gebrauch möglich sei, weil nach ihrer Auffassung die notwendigen Bedingungen in den fest vorgegebenen Konventionen aufzufinden sind. ${ }^{64}$

Genau dieses Verhältnis dreht die Dekonstruktion um. Der Grund ist schlicht, dass sich die Kontexte einer Äußerung weder abschließen noch kontrollieren lassen. Mit jeder Äußerung ist allein aufgrund ihres Auftritts eine neue Situation gegeben. Sie ist als aktuelles Ereignis in eine neue Lage versetzt, ebenso, wie sie zugleich eine neue Lage schafft. Der Sinn muss sich so also erst erweisen und setzt sich damit erst. Nicht die konventionelle Reproduktion ist daher der Normalfall, sondern umgekehrt die beständige Verschiebung des Sinns von Äußerung zu Äußerung. Anders kann es auch gar nicht sein, weil Konventionalität nicht die Voraussetzung von Sinn sein kann - vielmehr bedarf es erst des Verständnisses, um sehen zu können, inwieweit sich die aktuelle Äußerung in ihrem Sinn anderen angleicht. Derrida bezeichnet dies als die "Rezitierbarkeit von Äußerungen". Der schlichten Ableitung der Performanz aus dem Typ steht eine nicht beherrschbare Rekontextualisierung entgegen:

„Aufgrund seiner wesensmäßigen Iterabilität kann man ein schriftliches Syntagma immer aus der Verkettung, in der es gefasst oder gegeben ist, herausnehmen, ohne dass es dabei alle Möglichkeiten des Funktionierens und genaugenommen alle Möglichkeiten der ,Kommunikation' verliert. Man kann ihm eventuell andere zuerkennen, indem man es in andere Ketten einschreibt oder es ihnen aufpfropft. Kein Kontext kann es abschließen." 65

Mit jeder Äußerung ist das Zeichen, der Satz in eine neuerliche Umgebung gestellt und verschiebt seinen Sinn. Sinn schreibt sich so in Iteration fort und ist nicht im Vornhinein durch ein formelles Maß des Wiedererkennens festgelegt.

An diesem Punkt entzündet sich denn auch die Debatte zwischen Searle und Derrida. ${ }^{66}$ Dabei geht es ihnen nicht darum, dem Konventionalismus der Sprechakttheorie ein freies Flottieren von Performanz entgegenzusetzen, denn eine schlichte Umkehrung bestätigt nur die Hierarchie. Auch das Neue bedarf eines Maßstabs für den Vergleich. Darin sind sich Searle und Derrida einig.

63 Vgl. AUSTIN, Zur Theorie der Sprechakte (Fn. 46), 43.

64 WIRTH, Der Performanzbegriff (Fn. 39), 9 ff., 22 f.

65 JACQUES DERRIDA, Signatur Ereignis Kontext, in: DERS., Limited Inc., Wien 1988, 15 ff., $27 \mathrm{f}$. .

66 Siehe JACQUES DERRIDA, Limited Inc a b c, in: DERS., Limited Inc. Wien 2001, $15 \mathrm{ff}$; JOHN R. SEARLE, Reiterating the Differences, in: Glyph 2 (1977), $199 \mathrm{ff.} \mathrm{Dazu} \mathrm{FRANK,}$ Was ist Neostrukturalismus? (Fn. 58), $504 \mathrm{ff}$. 
Derrida dreht aber den Standpunkt Searles um seine eigene Achse, indem er die Konvention vom Grund zum Ziel der Erklärung macht. Searle scheint dies eine verkehrte Welt, eine "world upside down" $67 \mathrm{zu}$ sein, doch Derrida beabsichtigt damit nichts anderes, als die zuvor vom Logozentrismus auf den Kopf gestellte Welt wieder auf die Füße zu stellen. Die seriöse, "eigentliche", nicht-fiktive Rede sei nur ein theoretisches Konstrukt:

„Die Sprechakttheorie abstrahiert von der Erfahrungswirklichkeit umgangssprachlicher Verständigung so lange, bis sie eine endliche Menge von Wesenheiten und Gesetzen erfasst hat, die sie von ferneren Rücksichten auf konkrete Abweichungen des tatsächlichen Sprachgebrauchs - kurz: von jederlei Unregelmäßigkeiten - entbinden." ${ }^{\prime 68}$

Die von Searle marginalisierte Performanz wird von Derrida so ins Zentrum der Analyse gerückt.

Searle und Derrida stimmen darin überein, dass es so etwas wie Typen und auch eingefahrene Prozeduren geben muss, die den Sprechern Anhaltspunkte für ein Verständnis von Äußerungen geben. Sprecher und Hörer müssen davon ausgehen können, dass der andere die Regeln kennt und sich auch aller Wahrscheinlichkeit nach daran hält. ${ }^{69}$ Die Konsequenzen, die beide daraus ziehen, sind aber diametral entgegengesetzt: Während bei Searle die Konvention nicht nur Voraussetzung sprachlicher Performanz ist, sondern geradezu deren Möglichkeitsbedingung, ist sie bei Derrida der Effekt der Wiederholbarkeit. Bezogen auf das Verhältnis von Gesetz und Verfahren wäre bei Searle das Verfahren nur legitim als Repetition des Gesetzes, das heißt als identische Wiederholung; für Derrida dagegen wäre das Verfahren die Iteration des Gesetzes, welche nicht nur wiederholt, sondern auch verschiebt. Es geht also nicht um die Abschaffung einer der beiden Seiten, sondern um eine Akzentuierung.

Für Searle besteht zwischen der Äußerung und dem sie formierenden Typ ein Verhältnis logisch notwendiger Abhängigkeit:

"It is necessary to know that anyone who said it and meant it would be performing that speech act determined by the rules of the languages that give the sentences its meaning in the first place."70

Damit dies funktionieren kann, müssen die Regeln immer gleich bleiben. Wiederholbarkeit als Reproduzierbarkeit setzt stabile "Selbig-

\footnotetext{
${ }^{67}$ Siehe JOHN R. SEARLE, The world turned upside down, in: The New York Review 27 (1989), $74 \mathrm{ff}$.

${ }^{68}$ FRANK, Was ist Neostrukturalismus? (Fn. 58), 514.

${ }^{69} \mathrm{Zu}$ einem entsprechend koordinativen Konventionsbegriff siehe DAVID K. LEWIS, Konventionen. Eine sprachphilosophische Abhandlung. Berlin 1975.

70 JOHN R. SEARLE, Reiterating the Differences, in: Glyph 2 (1977), 199 ff., 202.
} 
keit"71 voraus: "Any conventional act involves the notion of repetition of the same."72 Ansonsten käme die ganze Konstruktion mit den Unwägbarkeiten der jeweiligen Äußerungssituation ins Rutschen.

Genau das ist aber, so Derrida, potentiell mit jeder Äußerung der Fall, denn die erneute Realisierung eines Aktmusters ist nicht einfach eine identische Durchführung, sondern erfolgt immer zeitlich verschoben. Genau diese Lücke aber gibt Raum für eine Sinnänderung. Der ganze mobilisierte Kontext kann sich mit dem Zeitablauf verändert haben. Searle unterwirft die sprachliche Performanz als identische Wiederholung vollkommen der Kompetenz; demgegenüber setzt Derrida sprachliche Performanz aufgrund der Wiederholbarkeit ohne Identität als Ereignis einer sprachlichen Aufführung frei, von der erst einmal zu entscheiden ist, inwieweit sie Vorhergehendes in sich aufnimmt und "zitiert" bzw. rezitiert.

Eine Wiederholung besteht aus zwei Aspekten. Sie unterscheidet den ersten vom zweiten Gebrauch und sie schiebt auf, indem sie die Verwendung auf zwei Zeitstellen verteilt. ${ }^{73}$ Wie kann man dann aber überhaupt noch von einer Wiederholung oder auch nur von einer Wiederaufnahme sprechen, was doch immer den Bezug auf ein Vorhergehendes als dem Aktuellen gleiches voraussetzt? Die Antwort, die Derrida gibt, steckt bereits in der Frage. Die Möglichkeit der Wiederholbarkeit gründet in der Distanz, die der Sprecher zu seiner Äußerung einzunehmen vermag. In der Äußerung distanziert sich der Sprecher von seinen eigenen Intentionen, weil er sie codiert. ${ }^{74}$ Ein Sprecher vollzieht also nicht einfach Performanz, sondern kann sich in der Äußerung von ihr distanzieren und sie verschieben. Das gilt auch für den Hörer, der den Sinn nicht nur aufnimmt, sondern ihn seinerseits auch $z u$ verschieben vermag. Der Sinn geht der Kommunikation nicht voraus, sondern wird erst in der Performanz geschaffen.

Aufgrund der Struktur von Wiederholung trägt jede Verwendung eines Sprachzeichens die Möglichkeit nicht kontrollierbarer Veränderung mit sich. ${ }^{75}$ Ganz wie Wittgenstein einmal sagte, dass die Sprache für sich selbst sorgen müsse, zieht sich sprachliche Performanz so an einem ihr als Sinneffekt eigenen Zopf von Konventionalität aus dem Sumpf einer sinnentleerenden Beliebigkeit. Die Sprache wird beständig durch das Sprechen verändert:

\footnotetext{
$71 \mathrm{Vgl}$. FRANK, Was ist Neostrukturalismus? (Fn. 58), 507.

72 SEARLE, Reiterating the Differences (Fn. 70), 199 ff., 207.

${ }^{73}$ Vgl. dazu Ralph Christensen, Was heißt Gesetzesbindung? Berlin 1989, 85 f., 101 f., 193 f., 198.

74 Siehe CHRISTENSEN, Was heißt Gesetzesbindung? (Fn. 73), $117 \mathrm{ff}$. und $129 \mathrm{ff}$.

75 Ebd., 142.
} 
"Sprechend differenzieren wir die Bedeutungen der Ausdrücke immer wieder aufs neue; alles ist in beständigem Fluß und die Identität der Bedeutung ist etwas rein Hypothetisches. ${ }^{776}$

Die einseitige Überordnung der Struktur über den Vollzug ist damit aufgelöst. Genauso wie sich beim Filmen das Drehbuch noch verändern kann, kann sich auch der Sinn des Gesetzes bei seiner Neuinszenierung im Verfahren verschieben.

\section{c) Iteration und Verkörperung}

Derridas Überlegungen zur Iterabilität haben sich für die Medien- und Kulturwissenschaften als überaus attraktiv erwiesen, da sie die Performanz von der Bevormundung durch ein ihr vorausliegendes Sinnzentrum befreiten und sie damit zugleich in ihrem produktiven Potential freisetzten. Die gegenwärtige kulturwissenschaftliche Beschäftigung mit der Performanztheorie hat daher das Konzept der Iteration schnell aufgegriffen und stellt sich letztlich als die Fortschreibung jenes Projekts dar, das Derrida am Ende von Signatur Ereignis Kontext formuliert hatte, nämlich "eine differentielle Typologie von Iterationsformen $\mathrm{zu}$ konstruieren ". ${ }^{77}$ Dies gilt für die Ritualtheorien, welche ihre Untersuchung auf die These von der mündlichen Wiederholung bestimmter Formulierung in bestimmten wiederkehrenden Situationen stützen, es gilt aber auch für die Theatralitätsforschung, welche die Inszenierung von Äußerungen im Rahmen der Alltagskommunikation und im Rahmen von Theateraufführungen als Praxis wiederholbarer Re-Zitation auffasst. Dabei tritt neben die Frage nach den Iterationsformen auch die Frage nach den wiederholbaren Verkörperungsbedingungen - ein Problem, das nicht nur die Theaterwissenschaft, sondern auch die GenderStudies und die Medientheorie betrifft.78

Damit aber rückt ein Aspekt in den Mittelpunkt, den schon Austin vernachlässigt hatte und welcher dann bei Searle gänzlich als kontingente Größe beiseite gestellt wurde: Die wieder am Performativen, am Vollzug und der Inszenierung interessierten Kulturwissenschaften fragen danach, wie Verkörperung Sinn zu tragen vermag, so dass dieser wieder erkennbar und rezitierbar ist. Rituale etwa leben von der präzisen Rekapitulierung des Vollzugs und gewinnen ihren besonderen Sinn aus ihrer Indexikalität. Die expliziten Performativa, die hier zum Einsatz kommen, werden dadurch realisiert, dass die Person hinter den

\footnotetext{
76 FRANK, Was ist Neostrukturalismus? (Fn. 58), 512.

77 DERRIDA, Signatur Ereignis Kontext (Fn. 65), 40

78 WIRTH, Der Performanzbegriff (Fn. 39), 9 ff., 34.
} 
Vollzug zurücktritt und so zu dessen Medium wird. Die Realisierung ist ,an strikte Repetition gebunden ". ${ }^{79}$ Eine Zeremonie hängt davon $a b$, dass an einer bestimmten Stelle ganz bestimmte Worte geäußert werden - auch wenn gleichbedeutende, anderslautende Ausdrücke zur Verfügung stehen. Der Vollzug ritueller Handlungen ist dabei in einem dreifachen Sinn performativ: erstens im Sinne Austins als Vollzug einer konventionalen Sprechhandlung, zweitens im davon völlig verschiedenen Sinn einer dramatischen Performance, in der die Teilnehmer verschiedene Medien benutzen und das Ereignis intensiv erfahren, und drittens im Sinne eines indexikalischen Wertes, den die Akteure während der Performance dieser zuschreiben und aus ihr ableiten. ${ }^{80}$ Deutlich wird dies auch an Theaterinszenierungen. Diese leben vom Zitieren von Handlungen. Sie transponieren die Handlungen auf die Ebene der Fiktionen und präsentieren sie zugleich. Indem der theatralische Akt die Handlung vorführt, zeigt sich sein Sinn, den wiederum die Aufführung verkörpert.

All dies legt eine Erweiterung des Performanzbegriffs nahe, welche sich dann auch im Wechselspiel zwischen Ethnographie, Soziologie und Theatralitätsforschung entfaltet hat. So hat die performative Ethnologie eine Re-Inszenierung ritueller Handlungen propagiert, die helfen soll, sich einen fremden kulturellen Rahmen mit seinen andersartigen Rollen, kollektiven Repräsentationen und Übergangszuständen zu erschließen. ${ }^{81}$ Die Handlungen fremder Kulturen, die sich dem Betrachter als Inszenierungen darstellen, sollen in der Re-Inszenierung durch den Ethnologen ihren besonderen Zeichencharakter zurückgewinnen. Die Ethnographie bringt so die rituellen Handlungen fremder Kulturen auf die Bühne der eigenen Kultur, wodurch rituelle Performative in theatrale Performances transformiert werden. Der Begriff der Performance bedeutet dabei nicht so sehr

„eine einzelne Tat als Handlung ausführen als vielmehr einen in Gang befindlichen Prozeß vollenden. Ethnographien als Theaterstücke aufführen heißt dann, uns ethnographische Daten in ihrer ganzen Fülle von Handlungsbedeutungen vor Augen führen." 82

Das spielerische "Auf-die-Bühne-Bringen" ist damit Voraussetzung für jeden ernsthaften Versuch des Fremdverstehens. Die Versetzung von

\footnotetext{
${ }^{79}$ Vgl. SIBYLLE KRÄMER, Sprache, Sprechakt, Kommunikation. Frankfurt am Main 2001, 143.

${ }^{80}$ STANLEY J. TAMBIAH, Eine performative Theorie des Rituals, in: UWE WIRTH (Hrsg.), Performanz. Zwischen Sprachphilosophie und Kulturwissenschaften. Frankfurt am Main 2002, $210 \mathrm{ff} ., 214$.

${ }^{81}$ Dazu VICTOR TURNER, Dramatisches Ritual, rituelles Theater, in: DERS., Vom Ritual zum Theater. Frankfurt am Main 1995, $140 \mathrm{ff}$.

82 TURNER, Dramatisches Ritual, rituelles Theater (Fn. 81), $140 \mathrm{ff}$.
} 
Äußerungen aus der Lebenswelt in die Theaterwelt wird nicht als Kontextwechsel, sondern als Perspektivenwechsel interpretiert, der als ReInszenierung sowohl das Verstehen der fremden als auch das Neuverstehen der eigenen Kultur ermöglicht.

Der ethnologische Performanzbegriff hängt sowohl hinsichtlich der rituellen Handlung im Kontext der fremden Kultur als auch deren ethnographischer Re-Inszenierung im Rahmen der eigenen Kultur von korporalen Aspekten ab. Diese stehen auch im Mittelpunkt der theatralen Performanzkonzepte, ${ }^{83}$ in denen sich der Akzent von der Ausführung auf die Verkörperung verlagert. Sichtbar wird dies in der Ersetzung des Ausdrucks Performanz durch den Ausdruck Performance. Theatralität wird nicht mehr mit den Mitteln einer Analyse des Performativen bestimmt, vielmehr bestimmt sich Performanz nun umgekehrt aus Theatralität, die diesem Begriff seine Kontur geben soll. Theatralität wird nicht nur sinnkonstituierendes Moment, wie es auch die Ethnographie des Alltags immer wieder beschreibt; ${ }^{84}$ sie gerät zum "allgemeinen kulturerzeugenden Prinzip" ${ }^{\prime \prime}, 5$ das auf die Performance als „Vorgang einer Darstellung durch Körper und Stimme vor körperlich anwesenden Zuschauern" abhebt. Dabei werden drei Aspekte sichtbar: Die Inszenierung als "spezifischer Modus der Zeichenverwendung in der Produktion", die Korporalität als Aspekt, der "sich aus dem Faktor der Darstellung bzw. des Materials ergibt", und schließlich die Wahrnehmung der dargebotenen Materialität als Aspekt, "der sich auf den Zuschauer, seine Beobachtungsfunktion und -perspektive bezieht", sind Kennzeichen dessen, was Performanz ausmacht. ${ }^{86}$

Mit dem theatralen Performanzkonzept wird eine Transformation von Austins Begriff des Performativen in einen allgemeinen Begriff der Performance vorgenommen. Die kulturwissenschaftliche "Entdeckung des Performativen" liegt demnach darin, dass sich alle Äußerungen immer auch als Inszenierungen, das heißt als Performances betrachten lassen..$^{87}$ Auch das Gesetz kann als Aufforderung zur Inszenierung verstanden werden. ${ }^{88}$ Es muss als Rechtsquelle im Verfahren inszeniert

${ }^{83}$ Siehe dazu ERIKA FISCHER-LICHTE, Grenzgänge und Tauschhandel, in: UWE WIRTH (Hrsg.), Performanz. Zwischen Sprachphilosophie und Kulturwissenschaften. Frankfurt am Main 2002, $277 \mathrm{ff}$.

${ }^{84}$ Siehe ERVING GOFFMAN, Interaktionsrituale. Frankfurt am Main 1996; sowie DERS., Rahmen-Analyse. Frankfurt am Main 1996.

${ }^{85} \mathrm{Vgl}$. WIRTH, Der Performanzbegriff (Fn. 39), 9 ff., 39.

${ }^{86}$ FISCHER-LICHTE, Grenzgänge und Tauschhandel (Fn. 83), 277 ff., 299.

${ }^{87}$ Vgl. WirTH, Der Performanzbegriff (Fn. 39), 9 ff., 39.

${ }^{88}$ Siehe auch WOLFGANG SCHIID, Richtersymbole. Zum Verfahren als "Theater des Rechts" im Mittelalter und Früher Neuzeit, in: GÜNTER GEHL/RUDOLF MEYER (Hrsg.), Leben in Mittelalter und Moderne. Weimar 2003, 11 ff. 
werden. Dies geschieht durch den konflikthaften Bezug der Parteien auf den gleichen Gesetzestext. Der Konflikt entzündet sich an der Lesart eines von beiden Parteien zugrunde gelegten Textes. Die streitenden Parteien suchen den Richter nicht auf als Hilfe für ihr stockendes Verständnis. Verstanden haben sie das Gesetz längst. Es geht ihnen um die Durchsetzung ihrer Lesart. Darüber soll der Richter entscheiden. Jede Partei versucht dabei, das Gesetz als Vorgabe für den Prozess so ins Spiel zu bringen, dass es für die eigene Position spricht. Das Gesetz wird damit von der Argumentation als Rechtfertigung verwendet und wird so zum Teil der Inszenierung eines Interesses. Die Gegenpartei versucht, diese Inszenierung zu stören und das Gesetz für die eigenen Interessen ins Spiel zu bringen. Daraus ergibt sich der Streit, den der Richter nicht nur entscheiden muss, sondern auch in seiner Urteilsbegründung darstellen und verwerten soll. Letztlich beruht jede Anwendung eines Gesetzes somit auf der Macht des Zitats: „Es ist die Macht dieses Zitats, die der performativen Äußerung ihre bindende oder verleihende Kraft gibt". ${ }^{89}$

\section{Medientheorie}

als Frage nach der Transkription von Sinn

Während die Sprachphilosophie die kommunikative Funktion der Sprechakte thematisiert und damit die funktionalen Bedingungen der Möglichkeit des kommunikativen Gelingens problematisiert hat, befassen sich die Kulturwissenschaften mit der Wirklichkeit der medialen Verkörperungsbedingungen. Damit ist endgültig die von Chomsky in die Linguistik eingeführte und von Searle in seiner Sprechakttheorie fortgeschriebene Verkürzung des Performanzbegriffs überwunden, die diesen der Differenz von Bezug und Anwendung, der Regelanwendung in Bezug auf ein System von Sprache oder Kompetenz unterworfen hatte. Bei der Performanz soll es sich hier noch um die Umsetzung von Strukturen bzw. Regularitäten handeln, die in der Kompetenz als dem anleitenden und maßgeblichen System für die Generierung sprachlicher Praktiken abgelegt sind. Die ganze Figur des Nachvollzugs gegenwärtig Vorgegebenen bleibt hier noch erhalten. Gegen sie richtet sich die dekonstruktive Kritik vom Performativen her. Es gibt in Zeichenketten keinen gegenwärtigen Sinn, den man nur zu exekutieren bräuchte.

89 So JUDITH BUTLER, Körper von Gewicht. Die diskursiven Grenzen des Geschlechts. Frankfurt am Main 1997, 309. Allgemein zum Zusammenhang von Iteration und Zitat hier auch DERRIDA, Signatur Ereignis Kontext (Fn. 65), 15 ff., 40. 
Mit der Hinwendung der Kulturwissenschaften zu den Verkörperungsbedingungen rücken aber auch die Aspekte der Medialität und Materialität performativer Akte in den Mittelpunkt des Interesses. Der so verstandene Performanzbegriff kann dazu beitragen, rechtstheoretische Probleme zu präzisieren. Auch hier lag das Verfahren lange im Dunkeln, weil man ihm als Erkenntnisprozess höchstens Defizite vorwerfen konnte. Während der Gerichtsprozess in den Nachbarwissenschaften als diskursive Maschine untersucht wird und für die Jurisprudenz wichtige, aber kaum rezipierte Erkenntnisse liefert, 90 bleibt das Verfahren als ureigenes Gebiet der Jurisprudenz in der Rechtstheorie weitgehend unbeachtet. Bezeichnenderweise sind es in die Wissenschaft gewechselte Praktiker, die als erste Berichte aus dem dunklen Kontinent geliefert haben. So hat Walter Grasnick die Werkzeuge der theoretischen Soziologie auf die Hauptverhandlung angewendet,91 Hans-Joachim Strauch das liegen gebliebene Programm der Rechtsprechungslehre wieder aufgenommen ${ }^{92}$ und Thomas Seibert den Justizdispositiv zum Brennpunkt der Rechtstheorie gemacht, ren akademische Vertreter überwiegend noch immer damit beschäftigt sind, die Semantik des logischen Positivismus oder die Hermeneutik der sechziger Jahre nachbuchstabieren.

Bei der Entdeckung dieses unbekannten Kontinents kann das Instrumentarium der Medienwissenschaften bisher kaum reflektierte Komponenten der gerichtlichen Praxis sichtbar machen. Jeder Praktiker weiß, dass ein Verfahren nicht nach Plan läuft, sondern immer auch Neues produziert. Diesem Ereignischarakter des Verfahrens fehlt bisher noch der theoretische Ort. Hier kann der in der Kulturwissenschaft entwickelte Performanzbegriff sinnvoll eingesetzt werden. Das Verfahren als Performanz des Rechts ist danach nicht länger die Exekution einer Struktur. Mit dem Akzent auf die Bedingungen der Verkörperung wird

${ }^{90}$ Siehe etwa THOMAS SCHEFFER, Materialitäten im Rechtsdiskurs. Von Gerichtssälen, Akten und Fallgeschichten, im vorliegenden Band, 349-376.

${ }^{11} \mathrm{Vgl}$. WALTER GRASNICK, Luhmann ante portas. Systemtheoretische Überlegungen zur Hauptverhandlung im Strafverfahren, in: ALBIN ESER/JÜREN GOYDKE/KURT RÜDIGER MAATZ/DIETER MEuSER (Hrsg.), Strafuerfahrensrecht in Theorie und Praxis. Festschrift fiir Lutz Meyer-Gossner zum 65. Geburtstag. München 2001, 207 ff.; WALTER GRASNICK, Unterwegs zu einer neuen Theorie des Rechts - Mit Luhmanntexten im Gepäck, in: DIETER DÖlLING/VOLKER ERB (Hrsg.), Festschrift fiir Karl Heinz Gössel zum 70. Geburtstag. Heidelberg 2002, $15 \mathrm{ff}$.

92 Siehe HANS-JOACHIM STRAUCH, Rechtsprechungstheorie. Richterliche Rechtsanwendung und Kohärenz, in: KENT D. LERCH (Hrsg.), Recht verhandeln. Argumentieren, Begrïnden und Entscheiden im Diskurs des Rechts. Berlin 2005, $479 \mathrm{ff.}$

$93 \mathrm{Vgl}$. dazu neuestens THOMAS-MiCHAEL SEIBERT, Gerichtsrede. Wirklichkeit und Möglichkeit im forensischen Diskurs. Berlin 2005. Untersucht wird hier nicht nur der Justizdispositiv, sondern auch die Frage: Wie man schafft, wovon man spricht. 
vielmehr der Ereignischarakter jeder Realisierung unabweisbar. Mit Hilfe der kulturwissenschaftlichen Performanzkonzepte ist daher die Wirklichkeit der Verkörperungsbedingungen des Rechts zu untersuchen, die maßgeblich von der Dynamik der Reproduzierbarkeit und der Iterierbarkeit bestimmt werden, also von "den stummen Prozeduren, der lautlosen Materialität der Medien, in denen unsere Sprachlichkeit sich vollzieht". ${ }^{94}$

\section{a) Verkörperung und Medialität}

Medien sind die Bedingungen des Verkörperns von Sinn. ${ }^{95}$ Die vorrangige Aufgabe des Mediums besteht darin,

"den ,Gegenstand' durch die Relation, die es zu ihm unterhält, an etwas Körperliches ,anzubinden'. ... Ohne Medium gibt es keine Bedeutung. Es ist die ,Schaltstelle', die zwischen einem, Gegenstand' und einem Interpretanten vermittelt, indem sie beide miteinander in Relation setzt. ... Es ist das

Medium, welches in seiner Materialität und durch den Prozess, als dessen Teil es seine Vermittlung erfüllt, diese flüchtigen Wesen an etwas Festes bindet." ${ }^{\prime \prime 6}$

Lange glaubte man, diese Bedingungen vernachlässigen zu können, weil sie als neutrale Vermittlungsinstanzen gesehen wurden, welche den transportierten Sinn unberührt lassen..$^{97}$ Mittlerweile gibt es aber einen weitgehenden Konsens darüber, dass Prozesse der Sinnkonstitution nicht unbeeinflusst von den Formen der materiellen Repräsentation von Sinn ablaufen. ${ }^{98}$

94 So SIBYLLE KRAMER, Sprache - Stimme - Schrift. Sieben Gedanken über Performativität als Medialität, in: UWE WIRTH, Performanz. Zwischen Sprachphilosophie und Kulturwissenschaften. Frankfurt am Main 2002, 323 ff., 332.

95 Vgl. dazu ALEXANDER ROESLER, Medienphilosophie und Zeichentheorie, in: STEFAN MÚNKER/ALEXANDER ROESLER/MIKE SANDBOTHE (Hrsg.), Medienphilosophie. Beiträge zur Klärung eines Begriffs. Frankfurt am Main 2003, 34 ff., 45; vgl. dazu außerdem SIBYLlE KRÄMER, Das Medium als Spur und als Apparat, in: DIES. (Hrsg.), Medien Computer, Realität. Wirklichkeitsvorstellungen und neue Medien. Frankfurt am Main 1998, $73,77 \mathrm{f}$.

96 ROESLER, Medienphilosophie und Zeichentheorie (Fn. 95), 34 ff., 45, 48.

97 Vgl. zu dieser Problematik SIBYLLE KRÄMER, Erfüllen Medien eine Konstitutionsleistung? Thesen über die Rolle medientheoretischer Erwägungen beim Philosophieren, in: MÜNKER/ROESLER/SANDBOTHE (Hrsg.), Medienphilosophie (Fn. 95), 78 ff., $80 \mathrm{f}$.

98 Dies gilt auch für sehr speziell motivierte und allgemein gefasste Medienbegriffe wie den von Luhmann, vgl. JOSEF RAUSCHER, Medialität und Medien, in: ERNST/ GROPP/SPRENGARD (Hrsg.), Perspektiven interdisziplinärer Medienphilosophie (Fn. 37), $25 \mathrm{ff} ., 31$ : „Der Grundunterscheidung von formalen Strukturgegebenheiten und materiellen Trägereigenschaften ... begegnen wir in nahezu allen Blicknahmen des Phänomens der Medialität. Dieser Unterscheidung entspricht in Strukturanalogie die Luhmann'sche Differenzierung von Medien als losen Kopplungen von Elementen (z. B. 
Weniger ausgeprägt ist der Konsens allerdings hinsichtlich der Frage, wie man sich die Einwirkung des Mediums auf Bedeutungsgehalte vorzustellen hat, und durchaus umstritten ist auch die Frage, ob, wodurch und in welcher Weise Medien die Denkformen des Menschen selbst determinieren. ${ }^{99}$ Diese Gemengelage von Einvernehmen und Streit kennzeichnet die Diskussion um die Medien, seit die Geistes-, Sozial- und Kulturwissenschaften ihre etablierten Gegenstände als durch und in Medienverhältnissen konstituierte neu zu begreifen beginnen. ${ }^{100}$

Auch wenn sich keine wissenschaftliche Disziplin mehr der medientheoretischen Reflexion verschließen kann, so ist damit noch lange nicht geklärt, was man denn unter Medien zu verstehen hat. Vielmehr gibt es eine Vielzahl von Medienbegriffen, die sich danach unterscheiden, ob sie eher die technische, die kommunikative oder die kulturkonstitutive Rolle von Medien in den Vordergrund stellen. ${ }^{101}$ Diese Begriffe können bei den philosophisch orientierten Medientheorien sehr elementar ansetzen, wie etwa die Unterscheidung Medium/Form, sie können sich aber auch am Alltagssprachgebrauch orientieren und unter Medien eben Zeitungen, Fernsehen, Internet usw. verstehen wie die empirisch orientierten Medienwissenschaften. ${ }^{102}$ Die Schwierigkeiten liegen natürlich zum einen darin, dass die Medienwissenschaft noch am Anfang ihrer Entwicklung steht, zum anderen liegen diese Schwierigkeiten aber auch in der Sache: Medien sind so unterschiedlich in unser Weltverhältnis eingewoben, dass es schwer fällt, sie auf einen bestimmten Begriff zu bringen.

Diese Situation in der Medientheorie lässt sich vergleichen mit der Situation in der Literaturwissenschaft Ende der sechziger Jahre, als man nach dem Scheitern von ontologischen Bestimmungsversuchen der Literatur an Verfahren des Textes wie Selbstthematisierung, Sprachthematisierung, Erzählthematisierung usw. anknüpfte, um Grade der Literarizität zu bestimmen: „Parallel zum semiotisch basierten

Geräusche) und Formen als relativ festen Kopplungen von Elementen (z. B. Schrift) Sibylle Krämer interpretiert dies mit Recht in Analogie zu Zeichenbedeutung/Zeichenträger."

${ }^{99} \mathrm{Vgl}$. dazu ERNST FISCHER, Medien-Sinn und Eigen-Sinn. Medienphilosophie in buchwissenschaftlicher Sicht, in: ERNST/GROPP/SPRENGARD (Hrsg.), Perspektiven interdisziplinärer Medienphilosophie (Fn. 37), 296 ff., 298 f.

${ }^{100} \mathrm{Vgl}$. dazu LAGAAY/LAUER, Einleitung - Medientheorien aus philosophischer Sicht (Fn. 40), $7 \mathrm{ff}$

101 Zur Diskussion und zum Vergleich der unterschiedlichen Medienbegriffe siehe LAGAAY/LAUER, Einleitung - Medientheorien aus philosophischer Sicht (Fn. 40), 7 ff., 21 ff., sowie KLOOCK/SPAHR, Medientheorien (Fn. 37).

102 Vgl. zur Verortung medienwissenschaftlicher Fragen zwischen den Disziplinen WERNER FAULSTICH, Einführung: Zur Entwicklung der Medienwissenschaft, in: DERS. (Hrsg.), Grundwissen Medien (Fn. 37), 11 ff., mit historischem Überblick. 
Literaritäts-Konzept ... könnte ein möglicher Weg auch für die Medientheorie darin liegen, zumindest probeweise nach spezifischen Verfahren von ,Medialität' zu fragen. Solche Verfahren müssten zwar ,unterhalb' der Ebene umfassend ausformulierter Theorien angesiedelt, aber dennoch so breit und sinnfällig im Material verankert sein, dass ihnen eine auch empirisch verifizierbare Signifikanz zukäme."103 Als solche grundlegenden Verfahren kommen in Betracht die Wiederholungsstruktur als verschiebende Einbindung des Ereignisses in Redundanzen, die Zeitstruktur als Zerdehnung der Kommunikation oder die Verkörperungsstruktur als Performanz von Sinn.

Das Moment der Wiederholung ist zentral für Medientheorien, welche die Auswirkungen des Mediengebrauchs auf das Bewusstsein thematisieren. Die Kritik an der Produktion weitgehend identischer Bewusstseinsformen durch die Kulturindustrie entwickelte sich in der Tradition der Frankfurter Schule. Auch für die an McLuhan anknüpfende Toronto School of Communication erwies sich dieses Moment bei der Analyse von Massenmedien wie Fernsehen als leitend, wenngleich hier die für die deutsche Tradition kennzeichnende Klage über den Verlust der Aura des Kunstwerks in der massenmedialen Wiederholung fehlte. Deutlich erkennbar ist, dass das Element der Wiederholung am Beginn seiner Begriffskarriere durchweg negativ als Repetition konnotiert war ${ }^{104}$ und erst in der heutigen Medientheorie als Verschiebung im Sinn von Iteration verstanden wird. ${ }^{105}$

103 ROLF PARR, Wiederholen, Ein Strukturelement von Film, Fernsehen und Neuen Medien im Fokus der Medientheorien, in: kultuRRevolution 47 (2004), 33 ff., 33.

104 Das Wiederholungselement im Sinne einer identischen Reproduktion ist grundlegend für die Kulturtheorie Adornos und Horkheimers, aber auch für die Medientheorie Benjamins, der der Wiederholung allerdings schon ein verschiebendes Moment einbaut. Die große Zahl empirischer Untersuchungen in diesem Bereich treibt den Übergang von der Repetition zur Iteration voran, indem sie zeigt, dass jede Wiederholung einen Kontextwechsel darstellt, vgl. HEIKE KLIPPEL/HARTMUT WINKLER, „Gesund ist, was sich wiederholt". Zur Rolle der Redundanz im Fernsehen, in: KNUT HICKETHIER (Hrsg.), Aspekte der Fernsehanalyse. Methoden und Modelle. Münster 1994, 121 ff.; ULRICH SCHMITZ, Postmoderne Concierge. Die ,Tagesschan'. Wortwelt und Weltbild der Fernsehnachrichten. Opladen 1990; WERNER HOLLY, Die Samstagabend-Fernsehschau. Zu ihrer Medienspezifik und ihrer Sprache, in: Muttersprache 102 (1992), 15 ff.; KNUT HICKETHIER, The Same Procedure. Die Wiederholung als Medienprinzip der Moderne, in:

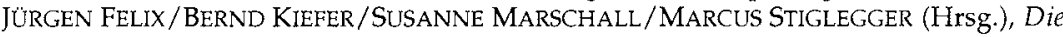
Wiederholung. Marburg 2001, $41 \mathrm{ff}$.

105 Siehe dazu GEORG CHRISTOPH THOLEN, Die Zäsur der Medien. Frankfurt am Main 2002 197 ff., mit der Analyse hybrider Performanzen wie Cross-Over, Infotainment/Edutainment und sonstiger unreiner Verkreuzungen. 
Die Zeitstruktur als Merkmal der Medialität ist dagegen zentral für die Leistung von Informationsübertragung und Informationsspeicherung. Sie wird hervorgehoben von Theorien, deren Schwerpunkt beim Begriff des Mediums und in der Mediengeschichte liegt. Dieses Element ist nicht nur für die Theorie Luhmanns wichtig, sondern gerade$\mathrm{zu}$ konstitutiv für den Medienbegriff Friedrich Kittlers. ${ }^{106}$ So ist etwa für Kittler ein Medium erst anzunehmen, wenn sich Daten auf der Zeitachse manipulieren lassen, also umgekehrt abgespielt werden können. Die menschliche Stimme wäre demnach kein Medium, wohl aber die Tonbandaufnahme.

Ein neuer Akzent in der Medientheorie ist die Hervorhebung der Performanz. Man will damit die Gesichtspunkte von Wiederholung und Zeitlichkeit verbinden. Sinn bedarf der Verkörperung. Er bleibt von der Verkörperung nicht unberührt, sondern wird dabei verschoben. Aus der Verbindung von Wiederholung und Zeitlichkeit ergibt sich die Performance als in der Wiederholung verschiebende Aufführung. ${ }^{107}$ Der Sinn wird in der erneuten Aufführung nicht einfach wiedergegeben, sondern umgeschrieben. Es werden damit Transkriptionsprozesse sichtbar, sowohl innerhalb eines Mediums als auch zwischen den Medien.

Die drei Momente von Medialität stehen dabei nicht im Gegensatz zueinander, sondern ergänzen sich. Es geht um eine Akzentuierung: Wiederholung steht im Vordergrund bei Kulturkritik, Zeitlichkeit bei Medienbegriff und Mediengeschichte, Performanz, wenn man erfassen will, was die Medien an konstitutiver Leistung erbringen. Medien transportieren nicht nur Sinn, sie sind konstitutiv für Sinn. Der Wechsel des Leitmediums vom Buch zum Computer rückt die Performanz in den Vordergrund. Dies zeigen die Inszenierungen virtueller Welten in den neuen Medien oder die Darbietung von Textflächen im Hypertext: Hier wird Sinn in Szene gesetzt, indem die Materialität der Zeichen und Symbole als Verkörperung von Sinn nach außen gekehrt wird. Die neuen Medien erlauben so die transformierende Rezitation durch den zum wahrnehmenden Betrachter gewordenen "Leser".

\footnotetext{
106 FrIEDRICH KITTLER, Geschichte der Kommunikationsmedien, in: JÖRG HUbER/ALOIs MÜLLER (Hrsg.), Raum und Verfahren. Düsseldorf 1985, 169 ff., FRIEDRICH KITTLER, Aufschreibesysteme 1800/1900, München 1989. Siehe dazu auch SIBYLLE KRÄMER, Friedrich Kittler - Kulturtechniken der Zeitachsenmanipulation, in: ALICE LAGAAY/ DAVID LAUER (Hrsg.), Medientheorien. Eine philosophische Einfiihrung. Frankfurt am Main 2004, 201 ff. Zu Luhmann vgl. THOMAS KHURANA, Niklas Luhmann - Die Form des Mediums, in: LAGAAY/LAUER (Hrsg.), Medientheorien (Fn. 40), $97 \mathrm{ff}$.

$107 \mathrm{Vgl}$. dazu die unter II.1.a angegebene Literatur sowie WINKLER, Diskursökonomie (Fn. 35), 215 ff., mit einer Warnung vor der Überschätzung dieser Kategorie.
} 
„Wir müssen die gewohnte Perspektive aufgeben, der zufolge der Sinn einer Botschaft durch ihren Kontext erhellt wird. Vielmehr sollten wir sagen, dass die Wirkung einer Botschaft darin besteht, einen Hypertext zu modifizieren, ihn komplexer zu gestalten, zu korrigieren und neue Assoziationen in einem kontextuellen Netz aufzubauen, das immer da ist. Das elementare Schema der Kommunikation wäre nicht mehr ,A übermittelt etwas an B', sondern ,A modifiziert eine Konfiguration, die A, B, C, D usw. gemeinsam ist'. Hauptgegenstand einer hermeneutischen Kommunikationstheorie ist also weder die Botschaft noch der Sender noch der Empfänger, sondern der Hypertext, der einer ökologischen Nische ähnelt, einem ständig in Bewegung befindlichen System von Sinnbeziehungen, die zwischen diesen Elementen bestehen. Gleichfalls sind die wesentlichen Operatoren dieser Theorie weder die Codierung noch die Decodierung oder der Kampf gegen das Rauschen durch die Redundanz, sondern jene molekularen Assoziations- und Dissoziationsoperationen, die die ewige Metamorphose des Sinnes bewirken."108

Wenn man vom Buch zum Hypertext wechselt, geht es nicht mehr um die Feststellung eines vorgegebenen Sinnes durch Lesen und Auslegung, sondern um die Verschiebung oder das Umschreiben des Sinnes durch Wiederverkörperung. Der Computer erzwingt den Übergang vom Lesen zur medialen Performanz.

\section{b) Medialität und Medienverstehen}

Will man zur Praxis gelangen, muss man sich von der klassischen Hermeneutik verabschieden. Texte führen keinen Sinn als gegenwärtigen mit sich. Der Sinn ist immer durch Kontexte aufgeschoben und umstritten. Damit entfällt die Steuerung der Performanz durch die Hinterwelt des Regelsystems. Der Vollzug verschiebt die Struktur; Performanz wird zur Performance. Die Anwendung wird zur Rezitation, die an nichts anderem bemessen werden kann, als dass sie wahrgenommen, erfasst und dadurch mit einem Eigensinn versehen wird. Das Dargebotene, die Figurierung von Zeichenketten in Texten oder die Konfiguration von Bewegungen zu Akten kann allenfalls Anlass für eine Leistung des Rezipienten sein. Dieser ist damit auch die produktive Instanz, die aus Wahrnehmung Vollzug generiert.

Die Rezeptionsästhetik hat dies treffend als eine Instruktionssemantik von Texten beschrieben. Text stellt sich im "Akt des Lesens" her, vollzieht sich als ein solcher Akt. Das dargebotene Zeichen entfaltet anleitende Wirkung, indem es anstelle einer amorphen Offenheit einen Cluster von Leerstellen bietet, die dazu einladen, hier mit Sinn

108 So Pierre LÉvy, Die Metapher des Hypertextes, in: Claus Pias/Joseph Vogl/ LORENZ ENGell/Oliver FAHLE/BRItTA NeItZel (Hrsg.), Kursbuch Medienkultur. Stuttgart 1999, 525 ff., 528 . 
einzuspringen und sich dabei zugleich den die Leere konstituierenden Rändern zu fügen. Lesen ist ein Füllen von Rahmen, das diese dadurch als solche ins Werk setzt. Wenn man also überhaupt von einer Regulierung des Verstehens sprechen will, dann kann dies allenfalls eine Selbstregulierung des Lesens durch dessen Vollzug meinen - Performance eben. Vom Text her gesehen tritt an die Stelle einer Bedeutung, die es durchzuführen gilt, eine Wirkung, die in Szene zu setzen ist.

Für das Problem des Erfassens von Text als Recht bedeutet dies, dass der juristische Leser nicht einen gegenwärtigen Sinn des Textes passiv erfasst, sondern aktiv fortschreibt. Der Akt des Lesens, aber auch der des Schreibens, hat keine andere Bedeutung als eben die, vor sich zu gehen. Damit gibt es auch keine weitere Instanz, auf die bedeutungsverleihend zu rekurrieren wäre. Gerade der Hypertext macht dies deutlich. Texte im Netz werden fortgeschrieben. Man kann nicht länger annehmen, dass man Elemente mit gegenwärtig vorgegebener Bedeutung manipuliert. Schon durch die Zusammenstellung der Textteile schafft man Sinn. Damit arrangiert man einen Vollzug von Verkörperungen, dem man durch sein Arrangement für sich Bedeutung zukommen lässt. Performance eben. Der Hypertext ist nichts anderes als eine „unabschließbare semantische Bewegung, in der die Identität des aufzufindenden Sinnes prinzipiell ,aufgeschoben' bleibt. "109 Wenn man sich nach alteuropäischem Verständnis auf einen ursprünglichen Sinn des Gesetzes berufen will, stößt man auf ein Paradox. Der Ursprung hat seinen Ort in der Nachträglichkeit von Sprache, denn Sprache erscheint erst mit der Performanz von Sinn. Erst in der Entscheidung von Recht hört die Verschiebung des Sinns vorläufig auf.

\section{c) Medienverstehen und Transkription}

Heute sieht man, dass der über das Gesetzbuch gebeugte Leser einen Schatten wirft. Genau in diesem Schatten liegen die für die Legitimität des Rechts entscheidenden Faktoren. Der Richter muss die Entscheidung nämlich nicht nur treffen - eine Entscheidung könnte ja auch anders getroffen werden. Er muss die Entscheidung vielmehr begründen. Aus dem Leser wird damit der Autor eines Textes. Die Souveränität des Richters als Autor ist aber eingeschränkt. Er muss in seiner Begründung den Bezug zum Gesetzestext wahren und die im Verfahren vorgebrachten Argumente verarbeiten. Im Medium des Gesetzbuches findet sich nicht der von der juristischen Hermeneutik gesuchte objek-

\footnotetext{
109 LUDWIG JÄGER, Transkriptionen: inframedial, in: CLAUdIA LIEBRAND/IRMELA SCHNEIDER (Hrsg.), Medien in Medien. Köln 2002, 123 ff., 124.
} 
tive Sinn, sondern es findet sich ein anderes Medium: die im Verfahren gesprochene Sprache. Auch dieses Medium findet seinen Sinn nicht in der aktuellen Äußerung, sondern im Hinblick auf ein anderes Medium: die Begründungsschrift. Der Richter liest nicht nur im Gesetz, er leitet ein Verfahren und schreibt am Ende eine Begründung. Dies alles verschwindet, wenn man das Recht ausschließlich unter dem medialen Paradigma des Buches begreift. Das Recht ist mehr als das Gesetzbuch. Es ist eine Medienkonstellation. Das Problem der Legitimation des Rechts liegt gerade im Zusammenspiel dieser Medien. Dabei sind Medien als dynamischer Vermittlungszusammenhang zu begreifen, in dem sich nicht nur etwas abspielt, sondern auch bestimmte Weichen gestellt werden. Wenn man eine Konstellation von Medien auf ein einziges Medium reduziert, droht man wichtige Weichenstellungen $\mathrm{zu}$ übersehen. Der Legitimationszusammenhang zwischen dem Gesetz als Text und dem Recht als Entscheidung wird damit zerrissen. Die Weichenstellungen können nicht mehr überprüft werden.

Wenn man nur das Gesetzbuch sieht, besteht die Performanz des Rechts allein im Nachvollzug des Textes. Die Verwirklichung des Rechts ist aber keine Lektüre, sondern ein praktisches Verfahren, dessen überraschende Wendungen sich der Prognose eines Lesers des Gesetzbuchs regelmäßig entziehen: so ist der jedem Richter bekannte Grundsatz "Wer einen Vergleich widerruft, verliert" in keiner Prozessordnung formuliert. Das Verfahren als Performanz des Rechts ist keine Ausführung des Gesetzes, sondern eine Inszenierung, in der sich immer wieder Neues ereignet. Seine Ausgangspunkte sind die Fallerzählung und die geltenden Normtexte. Das Verfahren organisiert einen Streit sowohl um die Fallerzählung als auch um die Lesarten des Normtextes. Die Normtexte gelten zwar, das ist unstreitig. Aber ihre Bedeutung für den Fall ist noch offen. Darum wird gestritten.

Die Aufgabe des Verfahrens ist es nun, die Geltung des Normtextes in eine Entscheidung zu überführen. Dabei wird die Geltung des Normtextes eingetauscht gegen die argumentative Geltung von Gründen für die gewählte Lesart. Den Normtext in seiner Geltung annehmen, um ihm jene Bedeutung zu verleihen, kann also nicht heißen, Letztere aus ihm herauszulesen. Vielmehr ist er als Legitimationsinstanz in Szene zu setzen. Mit dem Vorschlag, einen Normtext für das Verfahren zugrunde zu legen und ihn in einer bestimmten Weise zu lesen, wird dieser Normtext im Verfahren als Verkörperung von Recht sichtbar. Damit kann er mit anderen Lesarten besetzt werden; über die konkurrierenden Weisen, diesen Text zu verstehen, kann dann im Verfahren gestritten werden. Dieser Streit nimmt ständig auf den Normtext als Rechtsquelle Bezug und bringt für seine Verständnisweise Ar- 
gumente vor. Die Argumente bieten wiederum das Material für die richterliche Begründung des Urteils.

Die nötige Neustrukturierung der juristischen Sicht auf das Verfahren kann sich Unterscheidungen zu Nutze machen, die die Medientheorie im Begriff der Performanz entwickelt hat. Die Performanz von Texten ist danach von vier Momenten der Sinngenerierung durch Rezipienten gekennzeichnet. ${ }^{110}$ Es ist dies zum ersten die Bipolarität. Die Wahrnehmung des Textes ist keine passive Aufnahme durch den Rezipienten. Vielmehr wirkt dieser auf den Text zurück. Text als Ereignis von Sinn entsteht erst im Auge des Betrachters. Die Zeichenkette wird als Recht inszeniert, indem sie der Jurist als Normtext reformuliert. Das verweist auf das zweite Moment: die Korporalität. Das Wechselspiel von Wahrnehmung und Produktion braucht einen Halt. Diesen findet es in der Körperlichkeit. Der Vollzug von Text hat seinen Anknüpfungspunkt in der Materialität der Zeichen. Als materielle Zeichen bieten sie sich der Wahrnehmung dar und ermöglichen so die Umschrift in Bedeutung. Erst indem der Jurist dem Zeichenkörper des Normtextes Sinn zuweist, erzeugt er Recht. Diese Rechtserzeugung ist weder rein willkürlich noch von vornherein determiniert. Dadurch wird als drittes Moment der Ereignischarakter sichtbar: Recht wird erst im Moment der Entscheidung formuliert, und diese Formulierung könnte auch anders ausfallen. Die vorgebrachten Argumente der Parteien beeinflussen das Ergebnis des Verfahrens. Der kluge Richter lässt die Argumente der Parteien für seine Begründung arbeiten, so dass der Sinn von Recht nicht repetiert wird, sondern verschoben. Der Streit der Parteien wird also vom Recht nicht einfach entschieden, sondern dieser Streit zwingt das Recht in Metamorphosen. ${ }^{111}$ Darin liegt das vierte Moment der Transgressivität.

Die vier Momente der Sinngenerierung machen deutlich, dass die Darstellung von der Herstellung nicht $\mathrm{zu}$ trennen ist. ${ }^{112}$ Sprachliche Äußerungen bezeichnen nicht nur etwas, sondern vollziehen zugleich auch dasjenige, was sie bezeichnen. Während gewöhnlich das technische Erzeugen und das symbolische Darstellen unterschieden werden, hat die Performativitätsdebatte dazu geführt, die Konstruktivität und die Produktivität, das Machen und das Hervorbringen nun auch auf

110 Allgemein zum Folgenden SYBILLE KRÄMER, Was haben ,Performativität' und ,Medialität' miteinander zu tun? Plädoyer für eine in der, Aisthetisierung' gründende Konzeption des Performativen, in: DiES. (Hrsg.), Performativität und Medialität. München $2004,13 \mathrm{ff} ., 21$.

111 Siehe dazu LuHManN, Metamorphosen des Staates (Fn. 36) und auch Werber, Vor dem Vertrag (Fn. 36), 366 ff.

112 Vgl. dazu generell ERNST BLOCH, Experimentum Mundi. Frankfurt am Main 1975, 60 ff.: „Im erkennenden Fortbilden sind Erzeugen und Abbilden vermittelt.“ 
der Seite des Sprachlich-Symbolischen wirksam werden zu lassen, mithin das Darstellen nach dem Vorbild des Erzeugens zu denken. ${ }^{113}$ Dabei erweist sich vor allem das Moment der Transgressivität als kennzeichnend: Verstehen vollzieht sich als beständiger Übergang. Dieser Übergang wird aufgenommen vom Begriff der Transkription. ${ }^{114}$ Das lateinische transcribere steht für Abschreiben oder Umschreiben. Zwischen Abschrift und Umschrift ist der Spielraum des Ereignisses zu erkennen und damit das mögliche Moment der Transgression. Dieses Bedeutungspotential ist auch im Recht nützlich, denn der Sinn der Rechtsquelle wird auf dem Weg vom Gesetzbuch ins Verfahren zur Begründung langsam aber beständig umgeschrieben.

Vor seiner medientheoretischen Generalisierung wurde der Begriff der Transkription schon in anderen Wissenschaften verwendet und entwickelt, etwa in der Soziologie, wo man damit das Übertragen eines qualitativen Interviews in eine quantitative und auswertbare Form bezeichnet, und der Musikwissenschaft, in der man neben der Umschreibung von einer Notenschrift in die andere auch die Übertragung eines klingenden Werkes in eine Notenschrift als Transkription begreift. ${ }^{115}$ Grundlegende Bedeutung hat der Begriff aber vor allem in der Linguistik gewonnen: Während in der Phonetik damit die Übertragung einer Schreibung oder eines Phonems in eine andere als die ursprüngliche bzw. der jeweiligen Sprache entsprechenden Schrift bezeichnet wird, steht Transkription in der Konversationsanalyse für das Übertragen von gesprochener Sprache, Gesprächen oder auch Gebärden in eine schriftlich fixierte Form. Die deutschsprachige Gesprächsanalyse hat für diesen „Transfer von Mündlichkeit und Schriftlichkeit zu Zwecken empirischer Sprachanalyse" 116 das Verfahren der "halbinterpretativen Arbeitstranskription" (HIAT) entwickelt. ${ }^{117}$ Bei diesem Verfahren zur

113 KRÄMER, Was haben ,Performativität' und ,Medialität' miteinander zu tun? (Fn. 110), 13 ff., 25.

114 Dazu GeORG STANITZEK, Transkribieren. Medien/Lektüre: Einführung, in: LUDWIG JÄGER/GEORG STANITZEK (Hrsg.), Transkribieren. Medien/Lektïre. München 2002, $7 \mathrm{ff}$; LUDWIG JÄGER, Transkriptivität. Zur medialen Logik der kulturellen Semantik, in: LUDWIG JÄGER/GEORG STANITZEK (Hrsg.), Transkribieren. Medien/Lektïre. München $2002,19 \mathrm{ff}$.

115 KRÄMER, Was haben ,Performativität' und ,Medialität' miteinander zu tun? (Fn. 110), $13 \mathrm{ff}, 25$. Dazu hier STANITZEK, Transkribieren (Fn. 114), 7 ff.; JÄGER, Transkriptivität (Fn. 114), 19 ff. Als Quelle zu Folgendem http://de.wikipedia.org/wiki/Transkrip tion, samt Weiterverweisen.

116 ANGELIKA REDDER, Professionelles Transkribieren, in: JÄGER/STANITZEK (Hrsg.), Transkribieren. Medien/Lektüre (Fn. 114), 115 ff., 115.

117 Siehe KONRAD EHLICH/JOCHEN REHBEIN, Halbinterpretative Arbeitstranskription (HIAT), in: Linguistische Berichte 45 (1976), 21 ff. Dazu ANGELIKA REDDER, Professionel- 
Verschriftlichung mündlicher Dialoge wird Sinn aus nicht-wissenschaftlichen Kontexten zu wissenschaftlichen Zwecken umgeschrieben, wobei aber der Bezug zum Ausgangsmaterial trotz Verschiebung erhalten bleiben soll. ${ }^{118}$

Die Idee der Transkriptivität wird in der Medientheorie gegenüber der linguistischen Gesprächsanalyse generalisiert: Transkriptionen sollen nicht einfache Umsetzung in der Form sein, sondern produktiv den Text reformulieren. Dabei ist aber die Transkription als die Inszenierung von Sinn nicht vollkommen autonom; vielmehr wird sie an dem Anspruch gemessen, gerade diesen Ausgangspunkt $\mathrm{zu}$ artikulieren. Vorderhand scheint es sich beim Transkribieren erst einmal um eine Angelegenheit zwischen Medien zu handeln, um einen Transfer von Medium zu Medium. Möglich ist dies dadurch, "dass Medien vor allem andere Medien enthalten". 119 Dadurch verweist ein Medium aus sich heraus. Das Transkribieren vermag diesen Verweis aufzunehmen und zu vollziehen. Die Transkriptionsprozesse haben dabei den Charakter einer Transponierung ${ }^{120}$ oder einer Übersetzung. ${ }^{121}$ Wenn man mit Davidson davon ausgeht, dass "Übersetzen" bereits in der eigenen Sprache beginnt, so wird deutlich, dass Transkribieren auch schon ein inframedialer Vorgang ist. ${ }^{122}$ Transkribieren ist also eine Transformation, die den Ausdruck als Verkörperung von Sinn in Szene setzt und so auf diesen rückbezogen bleibt, ohne von ihm vollkommen festgelegt $\mathrm{zu}$ sein. Dies ist genau die Spannung zwischen der Bedeutsamkeit eines Normtextes dank seiner Geltung und seiner Bedeutung an Recht, die er im Urteil als Ergebnis des Verfahrens findet.

Bedeutungserschließung ist daher auf transkriptive Verfahren angewiesen, die es erlauben, „Projektionen aus dem Modus der Unbestimmtheit beziehungsweise Unlesbarkeit in den der Lesbarkeit zu

les Transkribieren, in: JÄGER/STANITZEK (Hrsg.), Transkribieren. Medien/Lektüre (Fn. 114), $115 \mathrm{ff} ., 129 \mathrm{ff}$.

${ }^{118} \mathrm{Zu}$ diesen Momenten des wissenschaftlichen Transkriptionsbegriffes ANGELIKA REDDER, Professionelles Transkribieren, in: JäGER/STANITZEK (Hrsg.), Transkribieren. Medien/Lektüre (Fn. 114), 115 ff., 115.

119 CLAudia LIEBRAND/IRMELA SCHNEIDER, Einleitung, in: LIEBRAND/SCHNEIDER (Hrsg.) Medien in Medien (Fn. 109), 9 ff., 9.

120 So werden bei der Gesprächsanalyse lautliche und gestische Artikulationen des Sprechers in eine Partitur von Graphemen übersetzt, wie etwa bei dem erwähnten Verfahren der halbinterpretativen Arbeitstranskription.

${ }^{121}$ Dazu ausführlich MICHAEL WETZEL, Unter Sprache - Unter Kulturen. Walter Benjamins "Interlinearversion“ des Übersetzens als Inframedialität, in: LIEBRAND/SCHNEIDER (Hrsg.), Medien in Medien (Fn. 109), 154 ff.

122 Dazu LUDWIG JÄGER, Transkriptionen: inframedial, in: LIEBRAND/SCHNEIDER (Hrsg.), Medien in Medien (Fn. 109), 123 ff., sowie SAMUEL WEBER, Transkribieren und "Einsprachigkeit", in: LIEBRAND/SCHNEIDER (Hrsg.), Medien in Medien (Fn. 109), 129 ff. 
versetzen. “123 Dieser Vorgang wird durch die Reflexivität von Sprache ermöglicht. Durch die Annahme der Lesbarkeit wird etwas zum Zeichen gemacht und dadurch weiteren Lesarten ausgesetzt. Dies ist auch die Situation des juristischen Verfahrens, in dem der Normtext den widerstreitenden Lesarten der Parteien ausgeliefert ist. Zur Auswahl einer verbindlichen Lesart bedarf es der Arbeit des Verfahrens, die aber insofern an den Normtext rückverpflichtet bleibt, als sie beansprucht, ihn in Szene zu setzen.

Das Transkribieren folgt dabei der medialen Logik einer Sinnerzeugung aus der Ausdruckswahrnehmung, die dadurch erst vollzogen wird. Der Vorgang des Transkribierens geht vom Präskript aus; dieses ist „das zugrundeliegende symbolische System selbst, das fokussiert und in ein Skript verwandelt wird" ${ }^{224}$ Im Aspekt der Wahrnehmung wird durch die Transkription der Text als Ausdruck buchstäblich vorgestellt. Text ist dabei in einem weiten Sinn zu verstehen, „der auch Bilder, Stimmen, Architektur usw. als Gegenstände von Lektüre zu konzipieren gestattet." $125 \mathrm{Zu}$ einem Ausdruck werden solche "Gegenstände" dabei überhaupt erst durch die Annahme einer Lesbarkeit, ${ }^{126}$ nicht durch irgendeine Eigenleistung, die bloß aufzunehmen wäre. Es ist der Verdacht von Sinn, der das Objekt damit zu einer der Gestaltung sich öffnenden Verkörperung macht. Diese Gestaltung vollstreckt die Transkription in Skripten als den „durch das Verfahren lesbar gemachten, das heißt transkribierten Ausschnitte(n) des zugrundeliegenden symbolischen Systems “.127 Man schreibt den Text als Gestalt in einen Sinn um. Dadurch verschwindet der Text im Verstehen. Den Status von Skripten erhalten Symbolsysteme nur dadurch, dass sie transkribiert werden, also aus Präskripten in semantisch auf neue Weise erschlossene Skripte verwandelt werden: "Tatsächlich stellt also jede Transkription die Konstitution eines Skripts dar, wiewohl das Verfahren zunächst auf ein schon vor seiner transkriptiven Behandlung existierendes symbolisches System trifft. ${ }^{\prime \prime 28}$

Es offenbart sich damit eine eigentümliche Beziehungslogik von Präskript, Skript und Postskript: obgleich das Präskript der Transkription

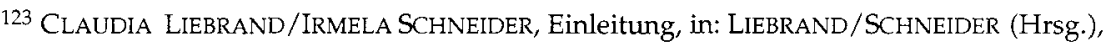
Medien in Medien (Fn. 109), 9 ff., 10.

124 JÄGER, Transkriptivität (Fn. 114), 19 ff., 30. Jäger spricht in seinen früheren Arbeiten meist noch vom "Quelltext" oder "Prätext", hat diese Begrifflichkeit aber im Weiteren zugunsten von "Präskript" aufgegeben.

125 STANITZEK, Transkribieren (Fn. 114), 7 ff., 8.

${ }^{126}$ Sonst werden sie als Unsinn abgetan. Vgl. dazu Oliver JaHraus, Systemtheorie, Dekonstruktion und Medientheorie. Wien 2001, 113

127 JÄGER, Transkriptivität (Fn. 114), 19 ff., 30.

${ }^{128}$ Ebd. 
vorausgeht, ist es als Skript doch erst das Ergebnis der Transkription. Man darf deshalb nicht davon ausgehen, dass zwischen Präskript/ Skript und Transkript ein einfaches Verhältnis der Abbildung besteht. So stellt die narrative Darstellung eines geschichtlichen Ereignisses als Transkript der in den Quellen dokumentierten, aber erst durch die Transkription narrativ selegierten und verbundenen Sachverhalte keine Abbildung dieser Sachverhalte dar, sondern konstituiert sie als historisches Ereignis. Die Aussage, dass sich die Geschichte erst im transzendentalen Rahmen des Wissens von ihr konstituiert, ${ }^{129}$ expliziert deshalb nichts anderes als die transkriptive Logik der Geschichtsschreibung: erst aus der Perspektive der darstellenden Transkriptionen der Quellen erhalten diese einen Status als Skript und damit eine Semantik. Die Quellen werden durch ihre narrativen Transkriptionen konstituiert und in einer bestimmten Hinsicht lesbar gemacht.

Die Transkription überschreibt den Text in einen geäußerten Sinn oder Gehalt. Sofern dieser als "geäußert" erzeugt wird, wird der Text auch schon wieder vernehmlich. Er steht wiederum als Ausdruck vor dem Sinn, dessen Vollzug somit jene Differenz aufreißt, die Generationen von Sprachtheoretikern Kopfzerbrechen bereitete: der Bestimmung des Verhältnisses von Signifikant zu Signifikat. ${ }^{130}$ Als Moment von Transkription betrachtet, wird die Sache an den ihr zustehenden Ort praktischer Verantwortlichkeit überwiesen. „Transkripte sind also nicht nur keine Abbildungen von Skripten, sondern diese sind ihrerseits auch nicht einfach Derivationen des Transkriptionsverfahrens. Tatsächlich kann man die Relation von Transkript und Skript nach dem Zeichen-Muster der Relation von Signifiant und Signifié verstehen: Beide lassen sich erst ex post actu - nach dem Verfahren der Transkription - als konstituierte Momente eines synthetischen Ganzen verstehen. Die Transkription konstituiert ein Skript und macht es lesbar, versetzt dieses jedoch zugleich in einen Status, aus dem sich Angemessenheitskriterien für den Lektürevorschlag ableiten lassen, den das Transkript unterbreitet." 131

Das Skript, welches jede Transkription erzeugt und durch das der Text als Verkörperung von Sinn vollzogen wird, geht in seiner Abhängigkeit von jener Transkription, der es seine Existenz verdankt, aber

\footnotetext{
${ }^{129}$ So MANFRed Riedel, Positivismuskritik und Historismus. Über den Ursprung des Gegensatzes von Erklären und Verstehen im 19. Jahrhundert, in: JÜRGEN BLÜHDORN / JOACHIM RITTER (Hrsg.), Positivismus im 19. Jahrhundert. Beiträge zu seiner geschichtlichen und systematischen Bedeutung. Frankfurt am Main 1971, im Anschluss an JOHANN GUSTAV DROYSEN, Historik. Rekonstruktion der ersten vollständigen Fassung der Vorlesungen 1857. Herausgegeben von Peter Leyh. Stuttgart 1977, 218, 236

130 Zur ausführlichen Kritik siehe JÄGER, Transkriptivität (Fn. 114), 19 ff.

131 Ebd., 19 ff., 33 f.
} 
keineswegs auf. Vielmehr sind Skript und Transkript immer schon auf Postskripte hin geöffnet, welche diese Differenz auf transkriptive Angemessenheit hin beobachten. Damit sind die Rahmenbedingungen eines rekursiven Spiels gesetzt, innerhalb dessen das erzeugte Skript eine Art Eigenrecht erlangt. ${ }^{132}$ Skripte sind in ihrer Behauptung als Lesart des Präskripts immer Postskripten geöffnet, die genau diese Behauptung thematisieren und konterkarieren, indem sie durch einen solchen Anspruch die Stelle des Skripts für sich einzunehmen gedenken. Das ist der Streit der Lesarten im Verfahren.

Wenn der Normtext durch den Leser vom Status einer Zeichenkette in Bedeutung überführt wird, entsteht ein Skript. Die Argumentation um die Vertretbarkeit von Lesarten eröffnet dann den Raum von Postskripten. Der Streit der Lesarten etabliert dabei das Skript als Rechtsquelle, wenn sich beide Parteien auf denselben Normtext beziehen. $\mathrm{Da}$ aber jede Partei den Bezug der anderen bestreitet, ist der Inhalt der Quelle noch nicht definiert. Die Quelle gibt nicht den Ausschlag, weil sie ein Maß für die streitigen Lesarten enthielte, sondern allein, weil sie gleichermaßen in den Transkriptionen als Skript enthalten ist.

Die "Tatsachen" liegen nicht als solche vor, sie sprechen nicht, ",sondern wir lassen sie sprechen". Dies geschieht im Rahmen einer Transkription, die dem Präskript den Status eines Skripts zuweist, so wie eine historische Überlieferung „erst durch unsere Fragen in eine Quelle verwandelt" wird. ${ }^{133}$ Dieser Vorgang geschieht allerdings nicht auf beliebige Art und Weise. "Weil es Postskripte gibt, die den Rekurs auf die Differenz von Skript und Transkript leisten, gilt vielmehr umgekehrt: ,Die Quellen haben ein Vetorecht."'134 Gerade dieser Aspekt ist für die Logik von Transkriptionsprozessen von konstitutiver Bedeutung: sie eröffnen einen Prozess des rekursiven Bezugs, in dem Transkripte auf ihr Recht, ihre Korrektheit, auf andere Möglichkeiten hin zu befragen sind. Zwischen Trans- und Postskript erweist sich die Kontingenz des Skripts. ${ }^{135}$

Transkription stellt also "ein grundlegendes Verfahren des Lesbarmachens kultureller Semantik dar, wobei die intramediale reflexive Doppeltheit der Sprache bzw. die intermediale Dualität der ins Spiel

132 STANITZEK, Transkribieren (Fn. 114), 7 ff., 10.

133 DROYSEN, Historik (Fn. 130), 218, 236.

${ }^{134}$ So STANITZEK, Transkribieren (Fn. 114), 7 ff., 10, im Anschluss an REINHART KOSELLECK, Standortbindung und Zeitlichkeit. Ein Beitrag zur historiographischen ErschlieBung der geschichtlichen Welt, in: DERS., Vergangene Zukunft. Zur Semantik geschichtlicher Zeiten. Frankfurt am Main 1979, 176 ff., $204 \mathrm{ff.}$

135 STANITZEK, Transkribieren (Fn. 114), 7 ff., 10. Zum Begriff des rekursiven Bezugs siehe WINKLER, Diskursökonomie (Fn. 35), 170. Im Unterschied zur Informatik bildet dieser Begriff hier keinen Gegensatz zur Iteration. 
gebrachten symbolischen Systeme oder Teilsysteme von entscheidender Bedeutung ist." ${ }^{136}$ Sinn ist nur in Transkripten zu haben und verdankt sich allein der Performanz von Verständigungsprozessen.

Mit den Kategorien von Gesetz als Präskript, Transkription als Lesart und Postskript als deren Thematisierung kann die Medientheorie einen Rahmen bereitstellen, innerhalb dessen die argumentative Dynamik und die Ereignishaftigkeit des Gerichtsverfahrens untersucht werden kann. Gleichzeitig bietet sie eine Grundlage, um die an den Richter gestellte Anforderung der Gesetzesbindung zu präzisieren, indem man ihre neue mediale Infrastruktur analysiert.

\section{Vom Gesetzbuch zum Hypertext des Rechts}

Stellt man die Frage nach den Medien des Rechts, so kommen neue Probleme in den Blick. Die Arbeit an Recht und mit Recht geschieht nicht allein in der Lektüre des Gesetzbuches, sondern im Vollzug des Verfahrens. Die Gleichsetzung des Rechts mit dem Gesetzbuch war schon immer eine Verkürzung, denn das Recht ist eine Medienkonstellation: es besteht aus Gesetzbuch, mündlicher Verhandlung und Urteilsbegründung. Dazwischen liegen Transkriptionsprozesse, da Juristen von einem Medium ins andere umschreiben - auch wenn sie das noch nicht reflektiert haben. Die Rolle des Gesetzbuches ist damit neu zu bestimmen. Es ist heute keine geschlossene Sinntotalität mehr, sondern funktioniert als Hypertext.

\section{Der Hypertext als Einlösung der juristischen Wunschkonstellation}

Relevante Informationen werden heute in immer größerer Zahl produziert, so dass der einzelne Arbeitende, der diese Informationen eigentlich zur Kenntnis nehmen müsste, von ihrer Fülle und der Geschwindigkeit ihres Erscheinens vollständig überfordert ist. ${ }^{137}$ Davon weiß jeder Jurist ein Lied zu singen. Man braucht nur für eine beliebige anstehende Rechtsfrage den Versuch zu unternehmen, anhand der den Normtext überwuchernden Kommentare sowie der unabsehbar streuenden Entscheidungen allen Spuren von Bedeutung nachzugehen, um recht schnell von der Vergeblichkeit dieses Unterfangens überzeugt zu

136 JAGER, Transkriptivität (Fn. 114), 19 ff., 35.

${ }^{137}$ Dies konstatierte Vannevar Bush, der wissenschaftliche Berater Roosevelts, schon im Jahre 1945: siehe BUSH, As we may Think (Fn. 20), 101. 
sein. ${ }^{138}$ Es scheint eine Überlastung des Einzelnen eingetreten zu sein, die mit den erprobten Mitteln nicht mehr zu beheben ist; gleichzeitig ist das Vertrauen abhanden gekommen, dass der gesellschaftliche Apparat und die objektive Vergesellschaftung dazu in der Lage sind, die einzelnen Aspekte der Arbeitsteilung auf rationale und effiziente Weise zu vermitteln. Neue, komplexe Probleme wie die ökologische Krise widersetzen sich einer arbeitsteiligen Lösung und zwingen dazu, die Dinge in einem neuen Typus von Zusammenhang zu denken. ${ }^{139}$ Es entsteht der Eindruck, dass sich die Probleme wie die Werkzeuge wesentlich dynamischer entwickeln als diejenigen, die ihnen gegenüberstehen. ${ }^{140}$ Das System der Arbeitsteilung überfordert die beteiligten Subjekte, und diese Überforderung bildet die wichtigste Schranke, die ein Weitervoranschreiten der Arbeitsteilung blockiert.

\section{a) Die Buchmetapher im Hypertext}

Angesichts dieser Lage ist es verständlich, dass nach einem Medium gesucht wird, das sich nicht mehr der vergeblichen Sammlung des Wissens verschreibt, sondern vielmehr verspricht, dessen Streuung sichtbar und dadurch unmittelbar greifbar zu machen. Als erster Ansatz zur Ausbildung eines solchen Mediums kann der von Vannevar Bush konzipierte Memex oder Memory Extender gelten. Der Memex war gedacht als „Arbeitsgerät zum persönlichen Gebrauch", das "eine Art mechanisierten privaten Archivs oder Bibliothek" darstellt: "Ein Memex ist ein Gerät, in dem ein Individuum all seine Bücher, Akten und seine gesamte Kommunikation speichert und das so konstruiert ist, daß es mit außerordentlicher Geschwindigkeit und Flexibilität benutzt werden kann. Es stellt eine vergrößerte persönliche Ergänzung zum Gedächtnis dar." ${ }^{.141}$ Dabei sollte sich der Memex allerdings völlig von dem her-

138 Siehe etwa die linguistische Analyse von $\$ 242$ StGB bei DIETRICH BuSSE, Recht als Text. Linguistische Untersuchungen zur Arbeit mit Sprache in einer gesellschaftlichen Institution. Tübingen 1992, $119 \mathrm{ff}$.

139 WINKLER, Theorie der zwei Köpfe (Fn. 26).

140 Siehe dazu GUNTHER ANDERS, Die Antiquiertheit des Menschen. Bd. 1: Über die Seele im Zeitalter der zweiten industriellen Revolution. München 1988, $21 \mathrm{ff}$.

141 Vannevar Bush dachte entsprechend dem damaligen Stand der Technik daran, den Memex auf der Grundlage von Mikrofilm-Dokumenten zu verwirklichen: „Das Problem der Masse wird durch einen weiterentwickelten Mikrofilm gelöst. Nur ein kleiner Teil im Inneren des Memex dient der Speicherung, der Rest lässt Platz für den Mechanismus selbst. ... Und es gibt die Möglichkeit zur direkten Eingabe. Auf der Oberfläche des Memex befindet sich eine transparente Fläche. Hier können handschriftliche Notizen, Photographien, Memoranden, alles Mögliche aufgelegt werden. Wenn dies geschehen ist, wird durch Hebeldruck eine Photographie angefertigt, die auf dem 
kömmlichen Verfahren des Katalogs lösen und eine ganz andere Dimension der Bewegung ermöglichen, welche letztlich in einen integralen Gesamttext münde, der aufgrund einer "assoziativen Indizierung" jederzeit aktuell verfügbar sei:

„Deren grundlegender Gedanke ist ein Verfahren, von jeder beliebigen Information - sei es Buch, Artikel, Fotografie, Notiz - sofort und automatisch auf eine andere zu verweisen. Dies ist es, was den Memex wirklich ausmacht: Es ist ein Vorgang, der zwei Informationen miteinander verbindet. ... Es ist genau so, als wären die jeweiligen Artikel, Notizen, Bücher, Photographien etc. leibhaftig aus weit entfernten Quellen zusammengetragen und zu einem neuen Buch verbunden worden. Und es ist noch mehr als dies, denn jede Information kann so zu einem Teil unzähliger Pfade werden. ... Ganz neue Arten von Enzyklopädien werden entstehen, bereits versehen mit einem Netz assoziativer Pfade, bereit, in den Memex eingebaut und dort erweitert zu werden. ${ }^{\prime 142}$

All dies klingt heute mehr als vertraut: Vannevar Bush beschreibt nichts anderes als den Hypertext. ${ }^{143}$ Gestützt auf den Computer als technisches Medium soll dieser nicht nur die arbeitsteilig differenzierten, zerstreuten und divergierenden Wissensbestände virtuell in einen Gesamttext integrieren, er verspricht auch, diese mit den generalisierten Wissensbeständen zusammenzuführen. Als ein Medium des Überblicks soll er das Spezialisierte, Partikulare mit dem Allgemeinen in Kontakt bringen und den zerstückelten Diskursen die Utopie einer Einheit entgegensetzen:

„Begriffe wie ,Docuverse' oder ,Infosphere' signalisieren den Anspruch, von der unüberschaubaren Pluralität noch einmal zum Singular, und vom Schwirren der konkurrierenden Medien und der textuellen Praktiken zu einem unifizierten Kosmos immer schon kompatibler Bedeutungen überzugehen." ${ }^{144}$

Was also an Schrift zuvor in das Buch eingeschlossen und mit diesem in der Bibliothek vergraben war, soll nun als Verweis über Hyperlinks sichtbar zur Hand sein. Die Bedeutungen brauchen nicht mehr mühsam erschlossen und ergründet $z u$ werden. Sie stehen stets für den "Zugriff" bereit. Kondensiert in diesen Zugriff ist so auch die „Grenze zwischen Innen und Außen" aufgehoben. ${ }^{145}$ Von der Seite des Textes her durch die Externalisierung aller Verweisungen, die ihn ausmachen,

nächsten leeren Segment des Memex-Films erscheint; dabei kommt das Verfahren der Trockenphotographie zum Einsatz", BUSH, As we may Think (Fn. 20).

142 BusH, As we may Think (Fn. 20).

143 Siehe dazu auch JAMES M. NYCE, From Memex to Hypertext: Vannevar Bush and the Mind's Machine. Boston 1991.

${ }^{144}$ WINKLER, Die Theorie der zwei Köpfe (Fn. 26).

145 Vgl. WINKLER, Docuverse (Fn. 3), 211. 
von der des Lesers, des "users", indem er sich den Text nicht mehr in einem inneren Semantisierungsvorgang erstellen und vorlegen muss; vielmehr entäußert er sich - die Rede von der "Website" und ihrem "Besucher", vom "Enter" machen dies klar - im Moment des Mausklicks durch das Eintauchen in den Text. Entsprechend enthusiastisch wird denn auch der Aufbruch allen Textes gefeiert:

"Hypertext macht explizit, was lineare Schriften noch der hermeneutischen Arbeit auflasten: das Netzwerk seiner Referenzen. Während lineare Schrift suggeriert, ihre Ideen seien homogen organisiert, ermöglicht der elektronische Text eine Koexistenz verschiedenster Strukturen. Der gesamte hermeneutische Gehalt eines Textes ist in der Verzweigungsstruktur seiner elektronischen Darstellung manifest. ${ }^{\prime 146}$

Allerdings: so neu sind die Verhältnisse im Hypertext nun auch wiederum nicht. Juristen war von jeher die Lektüre als eine kompilierend produktive Arbeit abgefordert und ist nach wie vor ihr alltägliches Brot. Text ist Mittel, Medium und Metier der Rechtsarbeit. „Recht als Text ${ }^{\prime \prime} 147$ also, Rechtstext, Texte des Rechts. Der Wechsel vom Singular in den Plural hat es in sich. Schon die rechtliche Würdigung eines so unspektakulären Delikts wie einem einfachen Diebstahl ${ }^{148}$ stürzt den Juristen in ein Meer von Text, "soweit das Auge reicht". ${ }^{149}$ Es bleibt ihm nichts anderes übrig, als die Sisyphusarbeit am Text immer wieder aufnehmen, durch die er seinerseits doch nur wieder den Kollegen dasselbe Problem bereitet.

„Er überfliegt und blättert durch, liest quer oder diagonal oder kursorisch, er folgt Querverweisen, verknüpft thematisch oder argumentativ verbundene Passagen über weite Einschübe hinweg, er lässt sich von Autor-Instruktionen vor- und zurückverweisen, nimmt nebenbei Fußnoten oder Anmerkungen, Marginalien oder Kommentare zur Kenntnis, verschafft sich einen Überblick durch Inhaltsverzeichnisse oder Stichwortregister, folgt den Lemmata in Enzyklopädien und Wörterbüchern in eigener Regie." 150

146 NORBERT BOLZ, Am Ende der Gutenberg-Galaxis. Die neuen Kommunikationsverhältnisse. München 1993, 222.

147 Dazu ausführlich aus linguistischer Sicht BUSSE, Recht als Text (Fn. 138), vor allem 259 ff. zum "Recht als textgebundene Praxis", aus rechtslinguistischer Sicht FRIEDRICH MÜlleR/RALPH CHRISTENSEN/Michael SOKOLOWSKI, Rechtstext und Textarbeit. Berlin 1997, 99 ff., aus Sicht der juristischen Methodenlehre FRIEDRICH MÚLLER/RALPH CHRISTENSEN, Juristische Methodik. 9. Aufl, Berlin 2004, Rn. 219 ff., $505 \mathrm{ff}$.

${ }^{148}$ Siehe die eingehende Analyse dieses Vorgangs durch BuSSE, Recht als Text (Fn. 138), $119 \mathrm{ff}$.

149 So allgemein der Spruch von ROLAND BARTHES, S/Z. New York 1974, 11.

150 ERNEST W. B. HESS-LUTTICH, Text, Intertext, Hypertext. Zur Texttheorie der Hypertextualität, in: JÜrgEN SÖRING/RETO SORG (Hrsg.), Andrö̈den. Zur Poetologie der Automaten. 6. Internationales Neuenburger Kolloquium 1994. Frankfurt am Main 1997, 53 ff., 61 f. 
b) Intertextualität

Das Verknüpfen von Texten kehrt den Grundzug der Intertextualität hervor, ${ }^{151}$ der im Hypertext besonders offensichtlich wird. Kein Text kommt aus dem Nichts. Kein Text steht für sich allein. Und letztlich dürfte kaum ein Text je wieder völlig verschwinden. Zusammen- und gegenseitig am Leben gehalten werden sie durch ein vielschichtiges Geflecht von Anspielungen, Verweisen, Bezügen und Traditionen. Kurzum: im "Raum eines Textes überlagern sich mehrere Aussagen, die aus anderen Texten stammen und interferieren. ${ }^{\prime 152}$ Dies mag zwar an literarischen Texten besonders auffällig sein, da diese in der Regel auch ihr ganz bewusstes Spiel damit treiben; beschränkt darauf ist Intertextualität indes nicht:

„Die Ränder von Manuskripten und frühen gedruckten theologischen, juristischen und medizinischen Texten wimmeln von Glossen, die, wie die Anmerkung des Historikers, den Leser instandsetzen, sich vom polierten Argument zu denjenigen Texten zurückzuarbeiten, aus denen es entwickelt wurde und worauf es beruht. ${ }^{.153}$

Seit Jahrhunderten werden in Kommentaren Fäden von Verweisen über die Texteme juristischen Normierens und Entscheidens gespannt. Präjudizien, Berufungen und auch Widerlegungen nehmen in jeder juristischen Verlautbarung eine Vielzahl anderer in sich auf und empfehlen sich selbst wieder weiter zum Bezug für eine Fortschreibung des Texts von Recht. Die Juristen zeigen in der Umtriebigkeit ihrer Arbeit an Text lediglich überdeutlich, was eigentlich Text ausmacht.

„Jeder Text schreibt sich ein in ein intertextuelles Ensemble künstlerischer / kultureller / formaler / kanonischer / biographischer Konstellationen. Jedes Wort produziert Bedeutungen erst im Kontext der umgebenden sprachlichen Einheiten - alles Geschriebene ist ,Zitat': Entwendung gelesener Schriften." ${ }^{\prime \prime 54}$

All diese Verschwisterungen und Verschwägerungen mit anderen Texten trägt der Text nicht etwa bei sich. Sie müssen immer mehr oder weniger mühsam wiederhergestellt werden, wie überhaupt selbst der her-

151 Zum Überblick WOLFGANG HEINEMANN, Zur Eingrenzung des Intertextualitätsbegriffs aus textlinguistischer Sicht, in: JOSEF KLEIN/ULLA FIX (Hrsg.), Textbeziehungen. Linguistische und literaturwissenschaftliche Beiträge zur Intertextualität. Tübingen 1997, $21 \mathrm{ff}$.

152 JULIA KRISTEVA, Probleme der Textstrukturation. Köln 1972, 245.

153 ANTHONY GRAFTON, Die tragischen Ursprünge der deutschen Fußnote. Berlin 1995, $41 \mathrm{f}$.

154 HeIKO IDENSEN, Inter(-Text, -Aktion, -Net). Kollaborative Text- und Theorieproduktion in digitalen Diskursen ... aber: wo bleibt und was wird aus dem Autor?, unter http://www.uni-hildesheim.de/PROJEKTE/netkult. 
kömmlichste Text nicht von sich aus seine Bedeutung preisgibt, sondern immer nur ein Angebot für den Leser darstellt. 155 Weder Bedeutung noch Verknüpfungen sind dem Text inhärent. Die Instanz zur Herstellung all jener "Bezüge“, die ihn intertextuell ins Meer der anderen Texte eintauchen lassen, ist der Leser. ${ }^{156}$ Folglich ist der Text „eine Produktivität", ,"eine Textverarbeitung ", ,eine Intertextualität". ${ }^{157}$

Dabei ist der Begriff der Intertextualität zunächst selbst ein schillernder Begriff mit vielfachen Bezügen. Gemeinsam ist allen Konzepten von Intertextualität ein „sukzessives Abrücken von einem eher unreflektierten Textbegriff, jenem mehr oder minder autonomen Text, der einem Autor verpflichtet ist und eine einmalige, abgeschlossene und unveränderliche Sinngröße in Kommunikationsakten darstellt. " 158 Differenzen bestehen in den Ansichten darüber, was als textuelles Geflecht an diese Stelle zu setzen ist.

Prägend für das Konzept der Intertextualität waren vor allem die literaturwissenschaftlichen Arbeiten von Julia Kristeva. ${ }^{159}$ Für sie ist Intertextualität der Leitbegriff eines grundsätzlichen Wandels im Umgang mit literarischen Texten. Es geht nicht mehr um die immanente Sinnsuche für ein Werk, das von allen seinen Bezügen gelöst als ein geschlossenes zu betrachten wäre. An diese Stelle tritt vielmehr ein neues, dynamisches Verständnis von Textualität, das sich anlehnt an Prozessabläufe beim Kommunizieren mit ästhetisch geprägten Texten. ${ }^{160}$ Der Autor verliert damit seine zentrale Stellung, ist er doch lediglich Durchgangsstation zahlreicher Vortexte, die er letztlich nur bündelt. Dasselbe gilt für den Leser.

Damit tritt schon ein grundlegendes Merkmal von Intertextualität hervor: in seiner Bedeutung, seinem Sinn ist ein Text nicht mehr als der momentan durch die Lektüre festgeschriebene, semantische Schnittpunkt einer Fülle von Texten, die gewissermaßen in ihm als Brennpunkt kumulieren. Der Text versammelt so in sich ein "Konglomerat von Wissenssystemen und kulturellem Code", 161 welche die Lektüre aufzunehmen hat. Text ist also lediglich eine Momentaufnahme im

${ }^{155}$ Siehe hierzu vor allem WOLFGANG ISER, Der implizite Leser. München 1972; sowie DeRS., Der Akt des Lesens. München 1976. Des Näheren auch ULRIKE BERGERMANN, "Verkörpert" Hypertext Theorien vom Schreiben?, in: ZMM news. Zeitschrift des Zentrums für Medien und Medienkultur der Universität Hamburg 1997/98, 24 ff.

$156 \mathrm{Vgl}$. HESS-LÜTTICH, Text, Intertext, Hypertext (Fn. 150), 53 ff., 59.

157 Julia Kristeva, Zu einer Semiologie der Paragramme, in: Helga Gallas (Hrsg.), Strukturalismus als interpretatives Verfahren. Darmstadt 1972, $163 \mathrm{ff.}, 170 \mathrm{f}$.

158 HEINEMANN, Zur Eingrenzung des Intertextualitätsbegriffs (Fn. 151), 21 ff., 27.

${ }^{159}$ Siehe vor allem JULIA KRISTEVA, La révolution du langage poetique. Paris 1974.

${ }^{160}$ HeINEMANN, Zur Eingrenzung des Intertextualitätsbegriffs (Fn. 151), 21 ff., 23.

${ }^{161}$ Ebd. 
Fluss der jeweiligen Kultur. Dies gilt nicht nur für Literatur, vielmehr ist dies ein Grundzug von Textualität überhaupt. Texte sind Knoten, momentan geknüpft in das Gewebe der Produktion und Emergenz von Sinn. "The text is not an autonomous or unified object, but a set of relations with other texts. ${ }^{162}$

Es fragt sich aber, wie man überhaupt noch zu einem Textverständnis kommen kann, wenn doch offenbar alles am Text fließt. Die Antwort liegt in der produktiven Leistung des Rezipienten, der sich für seine Lektüre vom Text anleiten lässt, indem er die gelegten Spuren und Verweise aufnimmt. Der Text wird gleichsam als kartographische Anregung aufgenommen, Fäden durch eine von ihm bezeichnete Region kultureller Sinnproduktion zu ziehen, Pfaden der Kompilation von Bedeutungen zu folgen und Wege von Wissen abzuschreiten. Möglich ist dies, weil aufgrund seiner Intertextualität der Text keineswegs zu einem Nichts zerstiebt. Die Verweise, die sich ihm einlesen lassen, indem er sie in seinen Formulierungen suggeriert, führen als Anhaltspunkte wiederum $z u$ Texten, die als Haltepunkte gegen ein Ausufern und Zerstreuen der Bewegungen der Lektüre eingezogen werden können.

„Texte sind natürlich das Ergebnis kognitiver Prozesse. In ihnen bündeln sich die selektierten ,Vor-Texte' der Textproduzenten - zusammen mit anderen Wissenselementen - im Sinne von Autor-Intentionen; und von Rezipienten werden konkrete Textangebote der Textproduzenten mit Hilfe eben solcher ,Vortexte' selektiv verarbeitet als Rekonstruktion von Sinn. Dennoch aber bleiben die Texte die wesentlichen Festpunkte in Interaktionsprozessen; als Signale von latentem Sinngehalt fungieren sie als entscheidende Orientierungshilfe sowohl für Textproduzenten als auch für deren Partner beim Textverstehen. ${ }^{\prime 163}$

Intertextualität ist daher als Relation zwischen Texten zu verstehen, die der Leser erst herstellen muss.

Mit der Abkehr von der Vorstellung vom Text als vorgegebener Sinngröße kann dieser nur noch als Teil kommunikativer Prozesse, als Vollzug von Sinn, kurz: als Performanz gesehen werden. Daher kommt Texten in pragmatisch orientierten Kommunikationsmodellen grundsätzlich nur noch der Status von Variablen zu. Texte haben keine Bedeutung an sich, sondern immer nur relativ zu bestimmten Interaktionskontexten. ${ }^{164}$ Text ist damit stets das Ergebnis einer reproduktiven Schöpfung und produktiven Replikation. Die Lektüre arbeitet, indem sie die Bezüge, denen sich Text verdankt, vollzieht. Dies ist aber nicht

162 VINCENT B. LEITCH, Deconstructive Criticism: an advanced introduction. New York 1983.

163 HEINEMANN, Zur Eingrenzung des Intertextualitätsbegriffs (Fn. 151), 21 ff., 31.

$164 \mathrm{Ebd}, 21 \mathrm{ff} ., 27$. 
einfach eine identische Wiederholung von etwas schon Vorhandenem; die Lektüre, die repliziert und rezitiert, verschiebt den Text gerade dadurch, dass sie ihn wiederherstellen will. Stabil bleibt allein die Zeichenkette, deren Bedeutung erst in der Praxis der Lektüre hergestellt wird. Die Lektüre bewegt sich dabei allerdings nicht frei im Universum des Sinns, sondern hat durch Abarbeiten der gegebenen Interaktionskontexte Plausibilität zu begründen.

\section{c) Das Verschwinden des Buches im Hypertext}

Nahezu tausend Jahre lang hat das Buch der Lektüre einen sicheren Hafen geboten. Die Scholastik lehrte den Leser, die Zeile als Teil eines Absatzes zu verstehen und den Absatz als Teil eines Buches. ${ }^{165}$ Es wurde eine Hierarchie im Raum des Sinns errichtet, denn die Zeile wurde dem Absatz untergeordnet und der Absatz dem Ganzen des Buches. An die Materialität des Buches schloss eine neue Formatierung des Sinns an, der nun zur Totalität gerundet wurde. Das Lesen war fortan begrenzt von den beiden Deckeln des Buches und gerichtet auf die Sinnmitte des Textes. Diese Wahrnehmung von Schrift als Buch wurde durch die Druckerpresse noch verstärkt, sicherte sie doch die Unveränderlichkeit des in Bleilettern gegossenen Textes.

Mit dem Internet kommt die Metaphysik des Buches zu ihrem Ende. Jetzt wird offensichtlich, was im Buch implizit war und marginalisiert werden konnte: die Struktur der Schrift als Hypertext. Auch wenn Glossen, Kommentare, Verweise und Editierungen die Ideologie eines nachzuvollziehenden Sinnganzen im Grunde immer schon perforiert und unterlaufen hatten, konnte man dies noch für Randphänomene halten. Nun aber vollzieht sich der Ausbruch der Lektüre aus ihrer Gefangenschaft im Einband des Buches, das ersetzt wird durch eine dispergente Oberfläche, die das lineare Lesen unterminiert.

„Die Gutenberg-Galaxis hat sich im Medium Buch die Form ihrer Einheit gegeben. Der Kanon des Gotteswortes und das Buch des Menschen sind die auf einander verweisenden Modelle von Tradition. Den Tod Gottes und das Verschwinden des Menschen konnte das Buch nicht überleben - es hat sich wieder in ein textum aufgelöst.“166

Mit dem elektronischen Zeitalter und den neuen Medien hat ein Wechsel vom Buch zum Bildschirm stattgefunden. Durch den allem Sinn

\footnotetext{
165 Dazu und im Folgenden IVAN IlliCH, Im Weinberg des Textes. Als das Schriftbild der Moderne entstand. Frankfurt am Main 1991; ROGER CHARTIER, Lesewelten. Buch und Lektïre in der frïhen Neuzeit. Frankfurt am Main 1990; STEFAN HESPER. Schreiben ohne Text. Die prozessuale Ästhetik von Gilles Deleuze und Felix Guattari. Opladen 1994.

166 BOLZ, Am Ende der Gutenberg-Galaxis (Fn. 146), 192.
} 
gegenüber gleichgültigen binären Code hat auch das Alphabetische und Ikonische als Leitmedium ausgedient. Text ist alles, was aufleuchtet und im nächsten Frequenzmoment des Bildaufbaus schon wieder verflimmert ist $\mathrm{zu}$ neuerlicher Anzeige. Die dauernde Bewegung des Textes wird damit augenfällig.

Schreiben und Lesen werden deshalb nicht verschwinden. Ganz im Gegenteil: es wird mehr geschrieben, gedruckt, verteilt und gelesen denn je. Dies ist auch ein Effekt des Computers, der die entsprechenden Produktions- und Distributionsketten in einer Weise privatisiert und verbilligt, dass die herkömmlichen Instanzen und Agenturen des Buches nur unter größten Anstrengungen mithalten können. Was sich dramatisch wandelt, ist der Akt des Schreibens und Lesens selbst von einem privilegierten Verfassen und Anordnen zu einem beständig in sich oszillierenden Informationsdesign:

„Die neuen Texte befreien sich vom Korsett der Buchform und der Autorität des Autors; sie verzweigen und vernetzen sich unbegrenzt, um endlich $z u$ werden, was das lateinische Wort, textum' meint: ein Gewebe. Diese sogenannten Hypertexte brauchen keinen Autor, sondern einen SoftwareDesigner. ${ }^{\prime \prime 67}$

Die Kontinuität des Schreibens und Lesens kehrt dadurch bei gleichzeitigem Wandel der medialen Präsentation den Text als Performanz hervor.

Das Navigieren im neuen Medium des Hypertexts verlangt allerdings noch vertraute Anhaltspunkte. Deswegen bleiben Buch und Bibliothek als verblassende Metaphern auch im Internet zunächst erhalten und geben einen unfreiwilligen Hinweis darauf, dass sich abermals als Illusion und Enttäuschung erweisen dürfte, was derzeit noch als Aufbruch in die totale Transparenz gefeiert wird: die Hoffnung, der hermeneutische Gehalt des Textes werde nunmehr "aus seinem doppelt unheimlichen Sitz befreit, aus dem Dunkel der Köpfe und aus der Dispersion über die verschiedenen Deutungen" ${ }^{\prime 68}$ Was vorher im Buch beschlossen war, soll nun auf der Bildschirmoberfläche ins Auge springen. Schon die mindestens ebenso unheimliche und sich ständig weiter beschleunigende Vermehrung der Suchmaschinen und Linklisten in nur wenigen Jahren lässt aber das genaue Gegenteil befürchten, hat diese Entwicklung doch inzwischen ein Ausmaß erreicht, das ein Aufsuchen der Suchmaschinen mit Hilfe von Metasuchmaschinen nötig macht. Es ist daher eher zu vermuten, dass sich auch der Hypertext in die "Heimsuchung des Buches" teilt:

\footnotetext{
${ }^{167}$ NORBERT BOLz, Das kontrollierte Chaos. Vom Humanismus zur Medienwirklichkeit. Düsseldorf $1994,239$.

168 WINKLER, Docuverse (Fn. 3), 50.
} 
„Die Bücher verschwinden buchstäblich in der Bibliothek, das Buch geht im Buche wie ein Fluss im Meere zugrunde, d. h. in seiner exuberanten Multiplikation und Serialität, in der die Schrift als disseminierende, räumlich und zeitlich verstreuende Spur wiederkehrt. ${ }^{\text {"169 }}$

Wenn das Datenuniversum Universalität behauptet, so schließt es zumindest der Struktur nach an Hoffnungen an, die bereits für die klassischen Medien eine Rolle gespielt haben, tritt die Utopie eines unifizierten Kosmos des Symbolischen doch keineswegs zum ersten Mal auf. So erschien die Sprache noch der Aufklärung als ein transparentes Medium, das die Welt in ihrer Totalität erschloss und dem Denken keinen nennenswerten Widerstand entgegensetzte. Diese Vorstellung musste zusammenbrechen, als sich mit den neuen Bildmedien eine Alternative zur Sprache etablierte. Die Bilder erschienen als eine Spur des Realen, voraussetzungslos zugänglich und international verständlich; die Sprache dagegen war als willkürliche Vereinbarung entlarvt, die nicht beanspruchen konnte, die Einmaligkeit eines Ereignisses abzubilden, sondern nur das Allgemeine benannte. Die Sprache war gekennzeichnet durch eine Reihe gewaltförmiger Ausschlüsse, die Bilder dagegen schienen den unmittelbaren Zugang zur Einheit der Wirklichkeit zu eröffnen. Erst die heutige Flut der Bilder macht sie als Stereotypen kenntlich, welche die Einheit von Sinn und Wirklichkeit genauso wenig erreichen wie die Sprache. Der Wunsch nach Einheit war der gleiche, nur das Medium hatte gewechselt.

Wenn ein Wunsch wie dieser allen Enttäuschungen zum Trotz immer wiederkehrt, drängt sich die Vermutung auf, dass er seine Wurzel in einer durchgängigen Illusion hat. Auch für das Datenuniversum als der vorläufig letzten Medienkonstellation ist es daher angebracht, "die Zweifel, die die anderen Medien getroffen und in ihren Geltungsansprüchen empfindlich demontiert haben, nun gegen das Strukturprinzip als eine beschreibbare Konstellation illusionär-wunschgeleiteten Denkens $\mathrm{zu}$ wenden. ${ }^{\prime 170}$ Dies erfordert, sich von der Idee zu verabschieden, es müsse immer eine einzige Bedeutung geben, die allein für das Reale einzustehen vermag. Solange dies nicht geschehen ist, wird man auch mit der Zuwendung zu den neuen Medien nicht aus dem Teufelskreis der erneuten Frustration entkommen. Dies wird erst möglich, wenn man sich auf die Vielfalt der Texte und der Bedeutungen einlässt und die Synthese als ein Resultat anerkennt, das mit jeder neuen Analyse wieder infrage steht. Das Datenuniversum muss sich von

\footnotetext{
169 WeTZEL, Die Enden des Buches (Fn. 19), 32.

170 WINKLER, Die Theorie der zwei Köpfe (Fn. 26).
} 
der ebenso totalitären wie illusorischen Vorstellung lösen, es sei in der Lage, alle anderen Medien in sich aufzunehmen. ${ }^{171}$

Seine Parallele findet dies im Recht mit der Idee eines der Rechtskultur als letztem Zweck aufgegebenen idealen Gesetzbuches, das als geschlossene Kodifikation die verschiedenen Normen zusammenzwingt. Das alles Recht in sich beschließende Gesetzbuch sollte der sichere Garant einer die widerspruchsvollen Strebungen vereinheitlichenden Totalität des gesellschaftlichen Handelns sein. Von dieser Idee gilt es sich zu verabschieden. Dies aber kann nur geschehen, wenn man nicht länger gebannt in die Leere starrt, in der sich das Signifikat offenbaren soll: es geht darum, sich auch von der Idee des beherrschenden Blicks zu lösen.

Der Einschluss der Sprachen in die Mauern eines Turms, um auf seiner Spitze Gott zu erreichen, ist schon einmal gescheitert. Man tut daher gut daran, die vielen Sprachen in der einen eigenen anzuerkennen. Der Jurist hat auch für die Vielstimmigkeit der Ordnungen ein offenes Ohr zu haben; verschließen kann er es ohnehin nicht, wie die vergebliche Suche nach dem verlorenen Signifikat gezeigt hat. Juristisches Entscheiden ist stets semantische Arbeit an der Bedeutung sprachlicher Ausdrücke und geschieht immer aus der Mitte der Sprachen heraus. Die Arbeit des Juristen an Recht besteht in der Entscheidung von Bedeutungskonflikten. Diese Entscheidung ist mehr als eine Erkenntnis und kann nur durch einen Gewaltakt zur Sprache des Rechts deklariert werden, denn die entschiedene Bedeutung lag dem Text nicht schon immer zugrunde: sie wurde vom Verfahren geschaffen und besteht nur so lange, bis sie im nächsten Streit infrage gestellt wird.

\section{Vom passiven Verstehen zur aktiven Transkription}

Der Hypertext hebt die vom Buch her bekannte Triangulierung von Autor, Text und Leser auf, die nun ihre festen Rollen verlieren. An die Stelle der Auslegung des Textes tritt jetzt die Verknüpfung von Texten im Hypertext.

\section{a) Autor und Leser im Hypertext}

Der Hypertext besteht nicht aus einem einheitlichen, sukzessive zu rezipierenden, eben linearen Text: er ist ein Konglomerat oder Komplex

\footnotetext{
171 WINKLER, Die Theorie der zwei Köpfe (Fn. 26).
} 
aus Texten, die durch Referenzverknüpfungen verbunden sind. ${ }^{172}$ So gesehen stellt der Übergang zu der Realisierung von Text in den elektronischen Oberflächen keinen Quantensprung dar, vielmehr kehrt er nach außen, was im Inneren des Textes eigentlich immer schon angelegt war. Das ändert indes nichts daran,

"dass wir an der Schwelle zu einer neuen medialen Kulturstufe stehen, mit der die Prinzipien und Verfahren einer medialen Erschließung von Wirklichkeit durch Konzepte von Hypertext und Intertextualität neu organisiert werden." 173

Es geht also um einen medial bedingten Umbruch der Intertextualität, der das kulturelle Prozessieren von Sinn betrifft. Es wandeln sich die "menschlichen Verfahren zur Konstitutierung von Umwelt und Wirklichkeit", die Kultur ausmachen. ${ }^{174}$ Das Besondere am Hypertext gegenüber den ihm vorangegangen Kulturzuständen ist nun, dass mit ihm als computerrealisierten Medium ein kultureller Zustand erreicht wird,

„bei dem sowohl die Textspeicherung wie die Intertextualität zu anderen Texten der Kultur externalisiert werden, d. h. so wie der Barde alle wichtigen Texte seiner Kultur mental, die Bibliothek textual bereitgehalten hatte, so hält der Computer sämtliche relevanten Texte samt ihren möglichen Relationsstrukturen in seinem Speicher parat und kann die Intertextualität per Knopfdruck am Bildschirm konkret realisieren, indem er die verschiedenen verknüpften Texte gleichzeitig zur Verfügung stellt. ${ }^{\text {"175 }}$

Genau dies war auch die Vision des Schöpfers dieses Ausdrucks, Thomas H. Nelson. Sein Ziel war nichts Geringeres als

"die Verwaltung des gesamten Weltwissens über ein riesiges, computerunterstütztes Begriffsnetz, das den Zugriff auf die entsprechenden informationellen Einheiten gestattet. Durch die Möglichkeit der simultanen und kollektiven Bearbeitung eines Dokuments soll der tendenzielle Gegensatz zwischen Autor und Leser aufgehoben werden." ${ }^{\prime 176}$

Nelson definiert daher Hypertext als nicht-linearen Text. ${ }^{177}$ Hypertext stellt also keine sequentiell serielle Reihung von Elementen dar, sondern ",besteht" aus einer vorab nicht absehbaren Fülle von in sich wei-

172 SVEN F. SAGER, Intertextualität und die Interaktivität von Hypertexten, in: KLEIN/FIX (Hrsg.), Textbeziehungen (Fn. 151), 109 ff., 116.

173 SAGER, Intertextualität und die Interaktivität von Hypertexten (Fn. 172), 109 ff., 109.

174 Ebd., 109 ff., 110.

175 Ebd., 109 ff., 121.

176 RAINER KUHLEN, Hypertext - Ein nichtlineares Medium zwischen Buch und Wissensbank. Berlin 1991, 219.

177 ThOMAS HOLMS Nelson, Getting it out of our System, in: GEORGE SCHECTER (Hrsg.), Information retrieval: a critical view. Washington 1967, 191 ff., 195. 
testgehend autonomen Einheiten, die nur ein loses Gespinst von Segmenten bilden. Damit ist Hypertext zugleich modular: er ist ein Netzwerk, dessen Knoten multimediale Gehalte darstellen und dessen Fäden durch Links, also Verknüpfungen realisiert sind.

Diese Verknüpfungen existieren nur im Moment des Erscheinens von Hypertext. Sie sind nicht zwingend, sondern können jederzeit aufgelöst und neu gezogen werden. Damit fallen die Knoten in eine neue Konstellation. In diesem Sinne "gibt" es eigentlich auch nicht Hypertext. Es bietet sich vielmehr in jedem Moment lediglich eine Hypertextbasis dar. Anhand dieser muss Hypertext immer wieder erst durch die kompositorisch semantisierenden Aktivitäten seines Betrachters, durch Transformieren und Kommentieren zu einem flüchtig momentanen Ganzen kompiliert werden. Die Knoten, die die einzelnen Gebilde durch Verknüpfungen zu einem Text verweben, müssen immer wieder neu geschnürt werden.

Eine Strukturierung, gar eine Linearisierung des Hypertextes verdankt sich nur dem Moment der Lektüre. Diese ist damit kein rein rezeptiver Vorgang mehr. Die Segmente sind nicht linear angeordnet wie die Perlen einer Kette oder die Kapitel eines Buches, sondern in der Form eines Netzes, das mehrere Wege von Punkt zu Punkt ermöglicht. Der Rezipient bewegt sich nicht allmählich und auf vorhersehbarem Wege durch eine Textfläche. Er "springt" von Punkt zu Punkt, von Link zu Link und stellt sich so seinen eigenen Text zusammen. Hypertext ist kein Gegenstand, sondern ein in jedem seiner Momente notwendig produktiver Vorgang. Der Leser "macht" sich buchstäblich "seinen“ Text und schlüpft damit zugleich in die Rolle von dessen Autor.

Dies ergibt sich aus der Eigenart des Links, die zugleich das Prinzip von Hypertext ist. Dieser hat eine denkbar einfache Architektur. Ein Hypertextsystem besteht technisch gesehen aus nichts anderem als Knoten und Links. Knoten sind all diejenigen Objekte bzw. Informationseinheiten, die für eine Verknüpfung angeboten werden und geeignet sind. Als solche sind sie weitestgehend autark. Die Eigenständigkeit der Knoten macht die Modularität von Hypertext und damit die Variabilität von Verknüpfungen aus. Da die Ausgestaltung der Knoten inhaltlich nicht bestimmt ist, kann alles mögliche Ziel von Verknüpfungen sein - von einer schlichten Graphik, einem Fleck auf der Oberfläche bis hin zu komplexen und umfangreichen Dokumenten wie den Maastrichtverträgen oder der Bibel. Soll Information vermittelt werden, ist allerdings ein Minimum an Kohäsion erforderlich: die einzelnen Elemente müssen unter sich Verbindungen aufweisen, die sie als Einheit wahrnehm- und handhabbar machen. Dies gewährt ihnen semantische 
Autarkie, welche ihre Verknüpfung mit anderen Elementen ermöglicht. Zum Netzwerk wird das Konglomerat koexistenter Segmente erst durch die Verweise aufeinander, die ihnen beigegeben werden, eben durch Links. Links als Verweise setzen durch die einseitig von ihnen ausgehende und auf ein Ziel hin festgelegte Relation Informationseinheiten als Knoten in eine Beziehung, die allerdings erst durch den durch den Klick ausgelösten Sprung realisiert wird. Zugleich hält den Hypertext nichts anderes zusammen als allein der Augenblick dieses Sprungs.

\section{b) Die Aufhebung der Textgrenzen}

Die Freiheit zum Text, die Hypertext damit schafft, hat allerdings ihren Preis. ${ }^{178}$ Es droht Beliebigkeit und damit auch jene Orientierungslosigkeit, die für das Internet als das Syndrom des "Lost in Cyberspace" beschworen wird:

„Was sich aus der Vogelperspektive als freies Bewegen in einem faszinierenden Netz darstellen mag, ist aus der Sicht eines konkreten Benutzers dieses Netzes die Aufgabe, sich in dem Netz, das er nicht unmittelbar überschaut ..., zurechtzufinden, zu orientieren. ."179

Nichts ist der Lektüre vorbestimmt außer dem Material, auf das sie zugreifen kann. All die Links, die Hypertext im Moment der ihn produzierenden Lektüre zu einem flüchtigen Gespinst zu verweben helfen, ergeben sich nicht aus ihm. Links müssen gesetzt werden. Damit können zwar Pfade von Text vorgezeichnet werden. Ob, von wem und vor allem wie diese beschritten werden, bleibt aber dahingestellt. Kein Link, der dafür gesetzt ist, ist zwingend. Nichts kann dazu zwingen, einem Link zu folgen. Wenn aber nicht sicher einzuschätzen ist, wohin die Wege führen mögen, bewahrt nichts davor, abzuirren.

Andererseits steht aber auch nichts dem entgegen, sich seinen ganz eigenen Weg zu suchen. Aufgrund der rezeptionsabhängigen Variabilität der Bezüge ${ }^{180}$ ist im Hypertext immer auch eine Informationsselektion möglich, die gar nicht zum Strukturkonzept des Autors bei der Informationsvermittlung gehört, sondern alleine vom Anwender angestoßen wird. ${ }^{181}$ Man kann sich seinen eigenen Sinn machen, indem man

178 Vgl. auch HESS-LÜTnCH, Text, Intertext, Hypertext (Fn. 150), 53 ff., 68.

179 Vgl. STEFAN MÜNZ, Orientierung, Navigation, Lost in Hyperspace, http://www.idsmannheim.de/grammis/ht/htxt206.

180 Zur Variabilität auf der Ebene der Textorganisation auch ROBERTO SIMANOWSKI, "McDonald's of Education" oder: Technologie einer konstruktivistischen Weltsicht. Hypertext im Sprach- und Literaturunterricht, http://www.dichtung-digital.de/ 2000/Simanowski/10-Jan/1HT.htm.

$181 \mathrm{http} / / \mathrm{www}$.ids-mannheim.de/grammis/ht/htxt103. 
nach eigenem Belieben die Sprünge durch die Texteme wagt. Im dem Maße, in dem der Leser zum Produzenten seines Textes wird, schwindet auch der letzte Rest von Autorschaft: Die Aufgabe des Autors gleitet über zu der eines Kompilators, Transformators, Herausgebers, Kommentators. ${ }^{182}$ Er gerät zum bloßen Lieferanten, dessen Gut zum Spielball der Rezipienten wird. Hypertext ist bloßes Strukturierungsangebot. Er ist der Versuch, die holistische Struktur der Bedeutung, die der Text beschwören und besagen soll, außen anzuschreiben und auf eine Oberfläche zu streuen, auf der die Rezeption zur momentanen Erarbeitung jener Bedeutung treffen mag.

Seinen markantesten Ausdruck hat dies im Projekt des Wiki gefunden. ${ }^{183}$ Mit ihm werden auch noch jene im Interesse der Absicherung der Integrität von Webseiten künstlich eingezogenen Zugangssperren niedergerissen, die die Rezipienten aus deren Manipulation ausschlieBen sollen. Wikis sind im World Wide Web verfügbare Seitensammlungen, die von den Benutzern nicht nur gelesen, sondern auch online geändert werden können. Wie bei Hypertexten üblich, sind die einzelnen Seiten und Artikel eines Wikis durch Links miteinander verbunden, die Seiten lassen sich jedoch sofort am Bildschirm ändern. ${ }^{184}$ Das wohl spektakulärste Wiki-Projekt ist die Wikipedia, eine OnlineEnzyklopädie, die der beständigen Bearbeitung der Besucher unterliegt. ${ }^{185}$ Dabei wird im Dienste einer möglichst problemlosen Zugänglichkeit Wert darauf gelegt, dass die Teilnahme an Wiki dem Besucher keinerlei besondere Programmierkünste abverlangt, wie es etwa bei herkömmlichen Programmen zur Erstellung dynamischer Webseiten in Gestalt der Beherrschung einer spezifischen Programmiersprache der Fall ist. Für Wikis gelten lediglich schlichte, schnell erlernbare und online eingängig dokumentierte Prinzipien. ${ }^{186}$ Darüber hinaus wird es

182 HeIKO IDENSEN, Inter(-Text, -Aktion, -Net). Kollaborative Text- und Theorieproduktion in digitalen Diskursen ... aber: wo bleibt und was wird aus dem Autor?, unter http://www.uni-hildesheim.de/PROJEKTE/netkult.

183 Siehe dazu die vierteilige Artikelreihe ERIK MOLLER, Tanz der Gehirne, unter: http:// www.heise.de/tp/deutsch/inhalt/te/14736/1.html.

184 Siehe http://de.wikipedia.org. Der Name stammt von wiki, dem hawaiianischen Wort für schnell.

185 Die Wikipedia annonciert sich selbst auf ihrer Hauptseite: "Wikipedia ist eine mehrsprachige Enzyklopädie, deren Inhalte frei nutzbar sind und es für immer bleiben werden. Die deutschsprachige Ausgabe wurde im Mai 2001 gestartet und umfasst derzeit 202314 Artikel. Bei Wikipedia können alle ihr Wissen einbringen - die ersten Schritte sind ganz einfach! Auf unserem Projektportal gibt es weitere Hilfestellungen sowie Möglichkeiten zur Beteiligung", siehe http://de.wikipedia.org (Stand: Februar 2005).

186 In der Wikipedia wird beispielsweise aus der Eingabe „ein ,kursives' Wort" ein kursives Wort. Die Gesamtheit dieser Tags wird als WikiSyntax bezeichnet, und unter- 
den Besucher leicht gemacht, selbst ein Wiki zu etablieren: die entsprechenden Scripts stehen als Open-Source-Projekte frei zur Verfügung und lassen sich auch einfach installieren und unterhalten. Unter den inzwischen über 100 verschiedenen "Engines" zum Betrieb eines Wi$\mathrm{ki}^{187}$ gibt es inzwischen auch schon eher kommerzielle und in den Bereich eines Online-Projektmanagements vordringende. ${ }^{188}$ Es findet sich auch ein JuraWiki, das sich anbietet als „Forum für alle Juristen, die sich zu ihrem Fachgebiet, der Rechtswissenschaft und allem, was dazugehört, austauschen wollen. Aber auch juristische Laien sind willkommen." 189

Die Wikis gelten als "der Beginn einer neuen Internet-Ära",190 vollendet sich doch im Wiki das Konzept des Hypertextes. Das in Hypertext angelegte Prinzip „Jeder kann alles editieren" wird mit Wikis in die konkrete Praxis umgesetzt: unter jeder Seite befindet sich ein „EditText"-Link, der es erlaubt, den Text der Seite direkt im Browser zu bearbeiten. Dabei beschränken sich die Möglichkeiten der Besucher nicht darauf, vorhandene Seiten zu ändern und neue hinzuzufügen, auch können sie Verweise auf andere Seiten setzen und neue Verknüpfungen schaffen. ${ }^{191}$ Durch die Wikis wird damit Wirklichkeit, was die Theorie in Hypertext vermutet hatte. Nicht nur wird so jegliche Illusion von Sinnzentren obsolet, es macht auch letztlich keinen Sinn mehr, noch eine Differenz von Autor und Leser zu vermuten. ${ }^{192}$

scheidet sich je nach verwendeter Wiki-Software. Allen Dialekten ist jedoch zu Eigen dass sie sehr viel einfacher aufgebaut sind, als das ansonsten im World Wide Web verbreitete HTML. Diese Beschränkung auf das Wesentliche ermöglicht einer großen Gruppe von Menschen, insbesondere auch Computer-Laien, mit geringem Lern- und Schreibaufwand an diesem System teilzuhaben, http://de.wikipedia.org/.

187 Vgl. MÖLLER, Tanz der Gehirne (Fn. 183), Teil 1.

188 Dazu MORITZ SAUER, Wikis auf dem Vormarsch. Vernetztes Projektmanagement via Wiki, in: Internet intern 03/04, 31 ff. Zum System selbst http://comawiki.martignier. net.

${ }^{189}$ Unter http://www.jurawiki.de.

190 MOLlER, Tanz der Gehirne (Fn. 183), Teil 1.

191 „Um auch das Setzen von Verweisen auf andere Seiten zu ermöglichen, erfand Cunningham [auf den Idee und Realisation von Wiki zurückgehen] ein Schema namens CamelCase' (wegen der Großbuchstaben, die wie Kamelbuckel hervorstehen). Zeichenfolgen, die einen Großbuchstaben am Anfang und innerhalb der Folge enthalten - z. B. ,WikiWiki', ,DesignPattern' - werden als Verweise auf andere Seiten mit diesem Namen interpretiert. Existiert die Seite noch nicht, kann sie durch Anklicken eines kleinen Fragezeichens neben dem Link angelegt werden. Da man eine Seite erst auf einer anderen Seite eintragen muss, um sie anzulegen, ist sichergestellt, dass neue Seiten mit bereits im Wiki vorhandenen vernetzt werden." MÖLLER, Tanz der Gehirne (Fn. 183), Teil 1.

192 So lädt etwa das JuraWiki ausdrücklich ein: „Sie können das JuraWiki benutzen wie eine normale Website, $d . h$. einfach lesen und klicken. Durch die WikiWikiWeb-Technologie können Sie aber auch jede Seite dieses Systems selbst ändern. Bitte lesen Sie 
"Innerhalb von Minuten wird bei Wikipedia der gleiche Artikel editiert von einem Studenten aus Tokyo, einer Einzelhändlerin aus Köln, einem Gärtner aus Essex. Würde man die Aktivitäten sichtbar machen, so sähe Wikipedia selbst aus wie ein gigantisches neuronales Netz, in dem ständig die Synapsen unter Feuer stehen. ... Die Entwicklung hin zu einer noch massiveren Gleichzeitigkeit, einer noch intensiveren Vernetzung unserer Kultur scheint unvermeidlich." 193

\section{c) Von der Auslegung zur Aufpfropfung}

Die Textualität von Wiki ist die reine Performanz von Sinn. Der Hypertext kann nun mit Recht als "Text zweiter Stufe" bezeichnet werden, der das Resultat performativ-verknüpfenden Schreibens ist, das in einem bestimmten Rahmen vollzogen wurde. ${ }^{194}$ Was aber bleibt von einem solchen "Rahmen" und inwieweit ist Hypertext überhaupt noch Text, wenn die permanente Umschreibung, das ständige Flackern der Sprünge von Link zu Link und durch diese das permanente Oszillieren über alle Regionen hinweg jegliche Begrenzung als obsolet erscheinen lassen? Die Frage nach der performativen Rahmung von Hypertexten steht vor dem offensichtlichen Paradox, dass die spezifische Verknüpfungsform von Hypertexten, der Hypertext-Link, sowohl für das kohärenzstiftende Zusammenführen als auch - zumindest im Rahmen des Internet - für die abschweifende Dynamik des Hypertextes verantwortlich ist. ${ }^{195}$ Das bedeutet, dass Hypertext sein Außen immer wieder als Text in Erscheinung bringen kann; zugleich aber kann Hypertext darin nicht gehalten werden. Er ist darauf angewiesen, in Erscheinung zu treten; ansonsten bleibt er unfassbar. ${ }^{196}$

Die Dynamik von Hypertext ist gekennzeichnet durch eine nicht $z u$ begrenzende Abschweifungs- und Aufpfropfungsbewegung, die ihrer-

dann aber zuerst HilfeFürAnfänger. Empfehlenswert sind auch einige Editier-Versuche im sog. WikiSandKasten. Bitte beachten Sie unsere NutzungsBedingungen. Wenn Sie wollen, können Sie sich als BenutzerAnmelden."

${ }_{193}$ MÖLLER, Tanz der Gehirne (Fn. 183), Teil 4.

${ }^{194}$ So UWE WIRTH, Performative Rahmung, parergonale Indexikalität - Verknüpfendes Schreiben zwischen Herausgeberschaft und Hypertextualität, in: DERS., Performanz. Zwischen Sprachphilosophie und Kulturwissenschaften. Frankfurt am Main 2002, 403 ff., 404, im Anschluss an Genettes Definition von Hypertext als Text zweiter Stufe, vgl GERARD GENETTE, Palimpseste. Die Literatur auf zweiter Stufe. Frankfurt am Main 1993.

195 WIRTH, Performative Rahmung (Fn. 194), 403 ff., 405.

196 Besonders deutlich wird dies bei dynamischen Webseiten, welche die aktuell angezeigte Seite erst im Moment ihres Aufrufs erzeugt, indem sie die entsprechenden Inhalte aus einer Datenbank abruft und dem Browser zusammen mit allgemeinen Anweisungen zur Erstellung der Bildschirmgestaltung übergibt. Verlässt der Besucher die Seite, so zerfällt diese in ihre abstrakte Anweisungsstruktur. 
seits durch das Gleiten der Schrift als iterativer Struktur bestimmt ist. ${ }^{197}$ Demnach kann prinzipiell jedes Zeichen mit jedem gegebenen Kontext brechen und auf absolut nicht sättigbare Weise unendlich viele neue Kontexte zeugen. ${ }^{198}$

Genau das löst auch die Aktivierung des Links aus. Betrachtet man die Erscheinung auf der Browseroberfläche im Ganzen oder auch nur in relevanten Teilen als komplexes Zeichen, so gerät es mit dem Sprung in die Seite in nicht absehbaren Kontext; zugleich aber ändert sich auf diesem Weg die Umgebung des vom Besucher evozierten Textes. Wesentlich ist dabei, dass es keinen neutralen Bezugspunkt gibt, der sich als eine Konstante von Text in die Bewegung des Prozessierens von Content einbeziehen ließe.

„Das Fehlen des absoluten Verankerungszentrums führt zur Idee einer universalen Dezentrierung - welche zugleich die ,abdriftende Dynamik' des Internet ausmacht, das als hypertext incessant unentwegt wächst." 199

Auch die Gebundenheit des Links an einen "Anker" spricht nicht dagegen, darin eine Aufpfropfung zu sehen. ${ }^{200}$ Der als Anker bezeichnete Befehl, der die Verknüpfung zweier Seiten herstellt, ermöglicht entgegen seiner Bezeichnung keine fixierende Zentrierung; er liefert lediglich den technisch notwendigen Angriffspunkt für eine Mausaktion im Raster der Bildschirmoberfläche und ermöglicht so das Auslösen des Sprungs. ${ }^{201}$ Der Link ist daher im wahrsten Sinne des Wortes nur der Sprung - und als das essentielle Moment von Hypertext teilt er dessen ganze paradoxale Anlage eines abwesend Anwesenden. Die Anker sind immer auch Bestandteil des Knotens, aus dem sie verweisen. Damit ist die vordergründig paradoxe Situation geschaffen, dass beide Relata des Links als Ausgangspunkt der Relation gesetzt werden. "Für alle Verweise gilt, dass sie sowohl Ausgangs- als auch Zielpunkt haben

197 Siehe auch DERridA, Signatur Ereignis Kontext (Fn. 65), 15 ff., 26.

198 Ebd.

199 WIRTH, Performative Rahmung (Fn. 194), 403 ff., 415.

200 Anderer Auffassung ist WIRTH, Performative Rahmung (Fn. 194), 403 ff., 415, welcher die Ansicht vertritt, die allgemeine Bewegung der Aufpfropfung sei durch ihre Ankerlosigkeit ausgezeichnet, während die Funktion des Links gerade darin bestehe, eine sprunghafte Abschweifung mit Anker zu sein; er verkennt allerdings, dass die Auszeichnung von Content als Auslöser einer Verknüpfung eine rein technische Notwendigkeit ist, da in HTML kein Tag für sich operieren kann, sondern nur über einen Bestand teil von Content.

201 So lassen sich Links durchaus über Javascript setzen, ohne an irgendeinen Anker gebunden zu sein: sie werden dann durch einen "Eventhandler", also gebunden an ein Ereignis der Webseite wie das Laden oder Schließen, ausgelöst. Das zeigt, dass der Link nicht etwa in der Auszeichnung von Content als Anker "besteht". 
müssen.“202 Das liegt daran, dass der Anker gerichtet sein muss. Weder darf der Absprung fehlen - die Ausrichtung auf das Ziel hinge in der Luft -, noch darf die Adressierung fehlen - der Verweis führte so „ins Blaue“. So oder so bräche die durch den Anker gesetzte Vektorisierung von Text in Text in sich zusammen.

Aufgrund dieser notwendigen Doppelbödigkeit des Ankers nimmt er auch Teil an der ",kohäsiven Geschlossenheit" des Knotens; ${ }^{203}$ mehr noch, er trägt entscheidend zu ihr bei, indem das von ihm angezeigte Außen des anderen Knotens immer nur ein Innen des einen sein kann. Dies bewirkt letztlich das profane Mirakel einer durch die unsägliche Primitivität der Architektur von Hypertext in jedem Moment unabsehbar möglichen Variabilität und Überkomplexität. Mit anderen Worten: In der elementaren Schlichtheit der Bauform liegt die ungeheure Virulenz von Hypertext. Der Link ist ein Ruf in den Text, von dem nie feststeht, wer ihn erhört, nicht einmal, dass er erhört wird.

Die allägliche, leidvolle Erfahrung mit der Fehlermeldung "404 $\mathrm{Pa}$ ge not found" im Internet macht dies deutlich. Der Anker kann das Ziel nur als Adressierung setzen. Wer sich hinter der Adresse verbirgt, ist nicht ausgemacht. Dem Etikett kann jederzeit ein anderer Träger zugeordnet werden. Wenn die Erwartung, die der Anker weckt, erfüllt wird, dann ist dies eine Sache des Glücks und nicht des Geschicks, denn kein Verweis kann von sich aus zwingend sein. Der Link ist so, genauer gefasst, jenes durch den Anker anwesend gemachte Abwesende, ${ }^{204}$ das der Poststrukturalismus in jedem Zeichen erkannt hat. ${ }^{205}$ Die digressive Bewegung der Aufpfropfung ist daher durchaus mit der Dynamik hypertextuellen Verknüpfens zu vergleichen - allerdings nur dann, wenn man den Umstand mit in Betracht zieht, dass sich diese Aufpfropfung einem explizit performativen Sprungbefehl verdankt. ${ }^{206}$

Das Versprechen des Links, von Text zu Text zu kommen, erfüllt sich immer erst im Moment des Sprungs, in dem der Absprung bereits verschwunden ist. Das macht jede Lektüre unabsehbar. Die unbegrenz-

202 Vgl. OLIVER HUBER, Hypertext - eine linguistische Untersuchung, unter http:// www.huberoliver.de/2_2.html, 2.2.2.1.

203 Vgl. STEFAN MüNZ, n:m-Relation, kohäsive Geschlossenheit, http://www.ids-mann heim.de/grammis/ht/htxt209

${ }^{204}$ KARIN WENZ, Formen der Mïndlichkeit und Schriftlichkeit in digitalen Medien, in: Linguistik online 1 (1998), spricht generell davon, dass die "Leerstelle traditioneller Texte durch Sichtbarmachen intertextueller Bezüge im Hypertext trivialisiert wird."

205 Generell zu der Parallele GEORGE P. LANDOW, Hypertext and Critical Theory, in: DERS. Hypertext. The Convergence of Contemporary Critical Theory and Technology. Baltimore 1992, 1 ff.; sowie DERS., What is a Critic to Do? Critical Theory in the Age of Hypertext, in: DERS. (Hrsg.), Hyper/Text/Theory. Baltimore 1994, $1 \mathrm{ff}$.

206 WIRTH, Performative Rahmung (Fn. 194), 403 ff., 416. 
ten assoziativen Verknüpfungsmöglichkeiten eröffnen dabei gleichermaßen den Raum für digressive Abschweifung wie für enzyklopädische Zusammenführung. ${ }^{207}$ Ein Einhalt gebietendes Zentrum gibt es nicht. Hypertext ist reine Performance, die Aufführung von Sinn ohne Regisseur und Drehbuch. Hypertext ist noch nicht einmal gebunden an das elektronische Medium, wenngleich dies ihm die hervorragendste Möglichkeit gibt, zu sich zu kommen. Das Besondere des Hypertextes liegt weniger in seiner elektronischen Materialisierung als in seinem Verknüpfungsverfahren.208

Das Risiko der Vorstellung, dass Recht im idealen Gesetzbuch bereits vorhanden ist, liegt darin, einen unentscheidbaren Konflikt als schon entschieden zu behandeln. Tatsächlich entscheiden Juristen Konflikte, die nicht durch Wissen entscheidbar sind. Darin liegt die Gewalt der Entscheidung, aber auch die Chance der Verfahrensbeteiligten. Kein Medium kann diese Gewalt wegnehmen. Wenn man doch so tut, nimmt man den Verfahrensbeteiligten die Chance, den Ausgang der Entscheidung $z u$ beeinflussen. Daran ist auch letztlich die Vorstellung gescheitert, alles durch das Medium geschriebener Sprache erreichen zu können. Der Inhalt eines Mediums ist immer ein anderes Medium: in der geschriebenen Sprache des Gesetzes findet man die gesprochene Sprache des Verfahrens. Allein das Wechseln zwischen den Medien des Rechts und das daraus resultierende Spannungsverhältnis erlauben es, die Unentscheidbarkeit bis zum Urteil offen zu halten.

Recht ist Hypertext. Das "Gesetzbuch" treibt also einen Etikettenschwindel. Es ist eher eine Kollektion stark modularisierter Segmente, eine Paragraphensammlung eben. In den Normtexten sind die einzelnen Gliederungsteile bis auf die Satzebene herunter immer für sich allein verständlich, da keine Kohäsion zu benachbarten Abschnitten besteht. ${ }^{209}$ Ihre Produktion unterliegt permanenten Verschiebungen, Novellierungen, Ergänzungen und Tilgungen, ohne dass dem Gesetz als einem solchen etwas Einschneidendes geschieht. Das erfährt bereits der Jurastudent leidvoll jedes Mal, wenn die neue Lieferung zur Ergänzung seines "Schönfelder" eingetroffen ist und er sich der Mühe einer reorganisierenden Kompilation unterziehen muss.

Gesetzbücher, Verordnungen und Richtlinien sind letztlich Ansammlungen von durch Paragraphen- sowie Abschnittsbezifferung

207 WIRTH, Performative Rahmung (Fn. 194), 403 ff., 418.

208 ROBERTO SIMANOWSKI, „MCDonald's of Education“ oder: Technologie einer konstruktivistischen Weltsicht. Hypertext im Sprach- und Literaturunterricht, unter http:// www.dichtung-digital.com/2000/Simanowksi/10-Jan/1HT.htm.

${ }^{209}$ FRANK KRUGER, Nicht-lineares Information Retrieval in der juristischen Informationssuche, Abschn. 511a Modularisierung, http://www.jura.uni-sb.de/dissertationen/ krueger/diss-511a.html. 
und Betitelung etikettierten Knoten, die fallweise miteinander verknüpft werden. Die Nennung von Normtexten anhand ihrer Ortskürzel und Kennziffer in Kommentaren, Schriftsätzen, Entscheidungen und Abhandlungen löst einen Reflex des Nachschlagens aus, wenn man das Textem nicht schon abrufbereit im Kopf hat. Doch das ist erst der Anfang. Die Oberfläche des rechtlichen Textwerks ist durchzogen von einem Geflecht von Querverweisen und Bezügen, wie etwa "Fundstellen von Rechtssätzen", "Zitierungsketten über Aktenzeichen", "Fundstellen in der Literatur", "bibliographische Angaben von Einzelnormen". "Unabhängig von den spezifischen Verweisarten treten folgende Möglichkeiten von Querverweisen innerhalb und zwischen den Gruppen juristischer Informationsquellen auf, nämlich Verweise von Normen auf Normen; von Urteilen auf Urteile, Normen und Literatur; von Literatur auf Literatur, Normen und Urteile"; dabei kann man bei Normen und Urteilen „von intra- bzw. interhypertextuellen Verknüpfungen sprechen, bei Verweisen auf die Literatur dagegen von extrahypertextuellen Verweisen".210 Wenn man also "die Modularisierung in Knoten und deren Vernetzung" als die wesentlichen Bestandteile von Hypertext feststellt, ${ }^{211}$ dann ist das Recht zweifellos ein Hypertext par excellence.

In den Hypertext des Rechts aber muss der Jurist eintauchen, wenn er den Text kompilieren will, der ihm als Entscheidung eines Rechtsfalls oder auch nur als eine qualifizierte Rechtsmeinung dazu abverlangt wird. Dabei gerät das Orientierungsproblem zu einem Problem der gebändigten Produktion von Text. Aufgrund der Bindungen und Verpflichtungen, denen der Jurist unterworfen ist, ist er für sein Prozessieren von Text im Raum des Hypertextes zwangsläufig Leser und Autor zugleich. Er ist einerseits gehalten, seinen Text $z u$ "finden". Zugleich kann er aber nicht zu diesem finden, ohne ihn durch die Auswahl der Knoten und durch deren Verknüpfung gemäß den Anforderungen des Falls zu „erfinden". Letztlich hat er genau jenen Text zu erstellen, auf dem seine Entscheidung von Recht beruhen soll. Er befindet sich in dem Dilemma, sich erst die Regel schaffen zu müssen,

210 Was Krüger hier schon für das System "Juris" beschreibt, kann getrost auf Rechtstexte im Ganzen übertragen werden. Siehe FRANK KRÜGER, Nicht-lineares Information Retrieval in der juristischen Informationssuche, http://www.jura.uni-sb.de/dissertatio nen/krueger/diss.html, im Print Marburg 1997, hier http://www.jura.uni-sb.de/dis sertationen/krueger/diss-511a.html; http://www.jura.uni-sb.de/dissertationen/kru eger/diss-511b.html.

211 So FRANK KRÜGER, Hypertext fuer Juristen - Grundlagen und Probleme, http:// www.fask.uni-mainz.de/user/krueger/publ/jurht-einf.html. 
der er für seine Anordnung und Strukturierung von Text zu folgen hat. 212

Der Jurist ist für sein Navigieren im Hypertext des Rechts Steuermann und Kartograph zugleich, indem er Texteme aufhäuft und ihnen eine Ordnung einzieht. Damit er dem Kurs vom Normtext zum Fall folgen kann, muss er ihn selbst erst abstecken, indem er in seinen Verweisen die Marken und Zeichen setzt. Aufgrund der Gesetzesbindung hat er für seinen Text von Recht auf der einen Seite die entsprechenden Knoten aufzusuchen; soweit ist er lediglich Rezipient. Allein durch die Frage aber, welches die für eine Entscheidung des Falls einschlägigen Knoten sind, wird er zugleich Produzent. Die Antwort auf diese Frage, die sich im Text der Entscheidungsnorm jeweils niederschlagen soll, verlangt von ihm, jene Knoten in eine für den Fall bestimmte Konstellation zu bringen. Er hat für seine Navigation also vom Fall her ein System von Verweisen zu entwickeln, sie in einer den Fall betreffenden Weise $z u$ verweben. Bei dieser Arbeit ist er aber auch schon wieder Rezipient: Die Verpflichtung darauf, dem Fall gerecht zu werden, zwingt den Juristen, sich auf die Fülle von Text einzulassen, mit der ihn die Beteiligten am Verfahren konfrontieren, auf all die Vorträge, Einlassungen und Schriftsätze, die vorgebracht werden und die ihrerseits die Vernetzung zum Text der Rechtsfrage beanspruchen.

\section{Von der Ableitung zur Inszenierung von Legitimität}

Mit dem Übergang von der Auslegung der Texte zur Verknüpfung von Texten im Hypertext ändert sich im juristischen Handeln nicht nur ein äußerer Rahmen, sondern dieses Handeln selbst. Die grundlegenden Probleme müssen neu diskutiert werden. Das gilt vor allem für die wichtige Frage der Legitimität. Der Richter kann Legitimität nicht aus dem Gesetzbuch ableiten, sondern muss sie sich in seinem Tun verdienen.

Die richterliche Entscheidung kann nicht mehr als Performanz einer vorgeordneten Struktur ausgegeben werden: das wäre die alte ZweiWelten-Lehre, wonach hinter den Wechselfällen des Verfahrens die ewige Struktur des Gesetzbuches regiert. Sie kann aber auch nicht einfach als Performance hingenommen werden, sonst liefert man sich mit

212 Vgl. allgemein RALPH CHRISTENSEN/MICHAEL SOKOLOWSKI, Theorie und Praxis aus der Sicht der Strukturierenden Rechtslehre, in: Rechtstheorie 2002, $327 \mathrm{ff}$ 
diesem Begriff den Zwängen funktionierender Systeme aus. ${ }^{213}$ Jede Performance kann gut oder schlecht sein. Dies ist an ihren immanenten normativen Maßstäben zu überprüfen. Genau wie ein Regisseur nicht einen bunten Abend, sondern ein Stück inszeniert, hat auch der Richter nicht irgendein Verfahren zu inszenieren, sondern ein Verfahren, das zu einer legitimen Entscheidung führt.

Es geht also im Recht um die Inszenierung von Legitimität. Entscheidend ist dabei, dass das Urteil einen Halt im Gesetz findet. In der Art, wie dieser Halt konstruiert wird, unterscheiden sich die beiden grundlegenden Konzepte einer legitimen Performanz des Rechts. Nach dem monologischen Konzept findet das Recht seinen Halt in sprachlichen Argumenten: die Bedeutung der Rechtsquelle muss in der Entscheidung abgebildet werden. Nach dem diskursiven Konzept findet das Recht seinen Halt sprachlich in Argumenten: ausschlaggebend ist die Frage, wer die im rekursiven Bezug der Argumentation konstituierte Rechtsquelle besser nutzen kann.

\section{Das monologische Konzept: \\ Die Abbildung des Gesetzes}

Die Verfassung fordert eine demokratische Rückbindung richterlicher Entscheidungen an den Volkswillen. Diese Rückbindung soll nach dem monologischen Konzept durch die Sprache des Gesetzes erfolgen. Das Verständnis demokratischer Selbstcodierung wird dabei legalistisch verkürzt: Das Volk gibt sich im demokratischen Prozess Gesetze, und indem der Richter den Inhalt dieser Gesetze ausspricht und anwendet, kommt der Volkswille zum Einzelnen zurück. Man verlässt sich damit auf das Gesetz als Text. Das Verfahren und die dort vorgebrachten Argumente, der Richter und seine Ausbildung, die kritische Kommentierung der Praxis durch Wissenschaft und Öffentlichkeit spielen in diesem Modell allesamt keine entscheidende Rolle. Es ist allein der objektive Inhalt des Gesetzes, der sicherstellt, dass die Selbstcodierung des Volkes in der staatlichen Praxis einlösbar bleibt. Damit wird eine Sprache, deren Bedeutungsinhalte vollkommen durchsichtig und problemlos beherrschbar sind, zur Grundvoraussetzung rechtsstaatlicher Demokratie. Die legalistische Verkürzung der Demokratietheorie mündet so in eine idealistische Verkürzung der Sprachtheorie und verbindet sich mit ihr zur Ikone rechtsstaatlicher Demokratie, welche weit abgehoben und anschlusslos über der staatlichen Praxis schwebt.

213 Davor warnt nachdrücklich WINKLER, Diskursökonomie (Fn. 35), 222 ff. 
Wie kann man Legitimität aus dem Gesetzbuch ableiten? Nach dem monologischen Modell muss die Entscheidung dazu einfach den Inhalt der Rechtsquelle abbilden. Das Einfache der Abbildrelation kann sich in einem streitigen Verfahren allerdings leicht vermehren, denn im Zweifel werden beide Parteien behaupten, mit ihren Anträgen den Inhalt der Rechtsquelle richtig wiederzugeben. Dieses Problem wäre nur $\mathrm{zu}$ handhaben, wenn der Richter eine versionslose Beschreibung des Inhalts der Rechtsquelle anfertigen könnte.

Wenn man dies mediologisch reformuliert, wird deutlich, dass dabei starke Anforderungen an die Sprache als Medium gestellt werden müssen. Ausgangspunkt der Abbildrelation sind die Normtexte, welche im Gesetzbuch enthalten sind. So stellt der Nötigungstatbestand des $\$ 240$ StGB für die Entscheidung das Präskript dar, die Bestimmung des Inhalts des Gewaltbegriffs durch die Anträge der Staatsanwaltschaft (Gewalt bedarf keiner körperlichen Zwangswirkung) die Transkription. Der Abstand zwischen Präskript und Transkript wird durch die schon im Normtext als Rechtsquelle enthaltene Bedeutung überbrückt. Allerdings kann man die Beziehung zwischen Präskript und Transkript einer weiteren Thematisierung unterziehen: Von Gewalt lässt sich nur reden, wenn eine körperliche Zwangswirkung beim Opfer vorhanden ist. So entsteht ein Postskript als weitere Lesart des Normtextes. Dieses Postskript nutzt die schon etablierte Beziehung zwischen Präskript und Transkript parasitär aus.

Für die juristische Methodenlehre stellt sich das Problem, die ständige Parasitierung der Beziehung zwischen Quelle und Lesart einzudämmen. Aber auch der Richter muss als Voraussetzung eines Urteils einen Leitsatz entwickeln, der sich zwischen den konkurrierenden Lesarten des Gewaltbegriffs entscheidet. Die klassische Methodenlehre will dem Richter dadurch helfen, dass sie ihm ein Mittel an die Hand gibt, um das parasitäre Wuchern einer Vielzahl von Lesarten zu reduzieren. Dieses Mittel ist die wahre Bedeutung des Textes. Sie erlaubt es, eine Grenze zu formulieren, jenseits derer das vorgeschlagene Postskript illegitim ist. Die Ersetzung des Präskripts durch Skripte ist nur legitim, wenn sie die wahre Bedeutung des Präskripts widerspiegelt.

Wenn man die wahre Bedeutung des Textes kennt, kann man den Parasiten abweichender Postskripte ausmerzen: das ist die Strategie der Wortlautgrenze als semantischer Grenze des Gesetzesverstehens. Die Verschiedenheit der Postskripte soll einen Maßstab finden in der propositionalen Struktur als dem gemeinsamen gedanklichen Gehalt, der eine technische Lösung des Transkriptionsproblems erlaubt. Danach lässt sich immer ein Bedeutungsgehalt erkennen, der einem Präskript und seiner Umschreibung gemeinsam ist. Kontrolliert und sicher bei 
der Hand genommen wird die Transkription durch die von ihr unberührt bleibende gemeinsame Proposition.

Dies mag als einfache Erklärung einleuchten. Allerdings löst sich diese Plausibilität in Luft auf, sobald die ebenso bescheidene wie nahe liegende Frage gestellt wird, was denn nun diese Propositionen sind. Sind sie geistige, intentionale Entitäten, logische Konstrukte, mentale Bilder? Blitzschnell füllen sich die Regale der Bibliotheken. Um der gemeinsamen Proposition habhaft zu werden, muss sie jedenfalls formulierbar sein. Dies kann nur in einer Sprache geschehen. Die Annahme einer gemeinsamen Bedeutung geleitet also die Transkription keineswegs auf sicheren Bahnen zur wahren Bedeutung. Sie vermehrt die Bedeutungen, denn auch die Sprache der Propositionen will transkribiert und damit verstanden sein:

"Somit müsste ich eine dritte Sprache einführen, um die Wahrheit der Proposition, die von der ersten und zweiten transportiert wird, zu bestätigen. Doch diese Operation könnte nur mit der Einführung einer vierten zu den ersten dreien gewährleistet werden und so weiter." ${ }^{\prime 214}$

Die magische Sprache der Propositionen liefert dem Beurteilen von Postskripten daher kein sicheres Gerüst. Der Parasit wird lediglich unsichtbar gemacht.

Diese Invisibilisierung kann allerdings nur gelingen, wenn man Postskripte abschneidet und den anderen Verfahrensbeteiligten das Recht auf Sprache nimmt. Ein solches Risiko liegt in jeder Rechtsordnung. Selbst im Rahmen des weitestgehend rechtsstaatlichen Verfahrensrechts der BRD werden in politischen Prozessen dem Angeklagten, der seine Motive erläutern will, die Mikrophone abgedreht ${ }^{215}$ oder dem Sprecher einer Bürgerinitiative von einem Oberlandesgericht strafverschärfend vorgeworfen, dass er entgegen der Auffassung des Gerichts den Aufruf zu einer gewaltfreien Demonstration nicht als Gewalt begreift. Der Kampf gegen die von der Institution zur Verfügung gestellte Sprache, den vergeistigten Gewaltbegriff, den das Bundesverfassungsgericht mittlerweile zu Recht aufgegeben hat, ${ }^{216}$ die Wahrnehmung des

214 David B. Allison, Die Différance der Übersetzung, in: ALFRED HIRSCH (Hrsg.), Übersetzung und Dekonstruktion. Frankfurt am Main 1997, 375 ff., 382.

215 Vgl. Emilios A. CHRISTODOUlidis/SCOTT VEITCH, The Ignominy of Unredeemed Politics: Revolutionary Speech as Différend, in: International Journal for the Semiotics of Law 10 (1997), 141 ff., 150 ff. Am Beispiel der Baader-Meinhof-Prozesse in der Bundesrepublik wird hier die Unvereinbarkeit der Sprachspiele von Gericht und Angeklagten gezeigt.

216 Vgl. dazu die Großengstingen-Entscheidung des Bundesverfassungsgerichts BverfG, I, BvR 718/19/22/23 aus 1989, Beschl. v. 10.1.1995. Dazu RAlPH CHRISTENSEN/ MICHAEL SOKOLOWSKI, Die Bedeutung von Gewalt und die Gewalt der Bedeutung, in: FRIEDRICH MULLLER/RAINER WIMMER (Hrsg.), Neue Untersuchungen zur Rechtslinguistik. Berlin 2001, $203 \mathrm{ff}$. 
Rechts auf eine eigene Sprache wird damit zum Strafgrund.217 Politische Prozesse sind sicher nicht der rechtsstaatliche Alltag. Allerdings redet ein Strafrichter auch im Normalfall nur dann mit dem Angeklagten, wenn er etwas herausfinden will, ansonsten aber mit dessen Verteidiger. Der Rechtsunterworfene wird beurteilt durch eine Macht, welche nur die Sprache zulässt, die sie vorher als fest und unveränderlich definiert hat. 218

\section{Das diskursive Konzept: \\ Die Transkription des Gesetzes}

Wenn man davon ausgeht, dass im Verfahren Recht nicht angewendet, sondern erzeugt wird, scheint der Faden zwischen Volk und Gesetz, zwischen Rechtsstaat und Demokratie zu reißen. Diese Verbindung ist unter realistischer Einschätzung der tatsächlichen und sprachlichen Bedingungen neu zu knüpfen. Will man das Postulat rechtsstaatlicher Demokratie einlösen, ist der Prozess der Herstellung von Rechtsnormen im Rahmen einer Rechtserzeugungsreflexion zu reformulieren. ${ }^{219}$ Die Explikation praktischer Normativität hat an den Urteilsbegründungen anzusetzen und die impliziten Standards der Rechtsprechung sichtbar zu machen. ${ }^{220}$ Damit kann die Selbstbeschreibung des Systems der eigenen Praxis stärker angenähert werden.

Die Lösung dieses Problems verlangt einen Neuansatz, für den verschiedene Bezeichnungen vorgeschlagen werden: prozedurales Recht, ${ }^{221}$ mediales Recht, ${ }^{222}$ reflexives Recht ${ }^{223}$ usw. ${ }^{224}$ Die Rechtspre-

217 Das Urteil des OLG Frankfurt 1. StE 1/82 ist abgedruckt in A. SCHUBARTH (Hrsg.), Der starke Staat. Dokumente zum Prozeß. Frankfurt am Main 1983, 131 ff. Diskussion bei RAINER WIMMER/RALPH CHRISTENSEN, Praktisch-semantische Probleme zwischen Linguistik und Rechtstheorie, in: FRIEDRICH MÜLLER (Hrsg.), Untersuchungen zur Rechtslinguistik. Berlin 1989, 27 ff., 36.

218 Vgl. dazu CHRISTODOULIDIS/VEITCH, The Ignominy of Unredeemed Politics (Fn. 215), $141 \mathrm{ff} ., 149$.

219 Dazu schon FRIEDRICH MÜlLER, Fragen einer Theorie der Praxis, in: AöR 95 (1970), 154 ff., sowie DERS., Juristische Methodik und politisches System. Berlin 1976, 33 ff. zum Stichwort Explikation; FRIEDRICH MÜLLER/RALPH CHRISTENSEN, Juristische Methodik. 9. Aufl., Berlin 2004, Rn. $536 \mathrm{ff}$.

220 Siehe dazu MARIELE DEDERICHS/RALPH CHRISTENSEN, Inhaltsanalyse als methodisches Instrument zur Untersuchung von Gerichtsentscheidungen, vorgeführt am Beispiel der Rechtsprechung des EuGH, in: FRIEDRICH MülLER/ISOLDE BURR (Hrsg.) Rechtssprache Europas. Reflexion der Praxis von Sprache und Mehrsprachigkeit im supranationalen Recht. Berlin 2004.

$221 \mathrm{Vgl}$. dazu RUDOLF WIETHÖLTER, Entwicklung des Rechtsbegriffs, in: VOLKER GESSNER/GERHARD WINTER (Hrsg.), Rechtsformen der Verflechtung von Staat und Wirtschaft Opladen 1982, 38 ff.; DERS., Zum Fortbildungsrecht der (richterlichen) Rechtsfortbil- 
chung wird danach nicht mehr als Rechtsanwendung begriffen, sondern als Rechtsproduktion. ${ }^{225}$ Das Rechtssystem erscheint dann nicht mehr als Gesamtheit der Normen, sondern als Gesamtheit der Handlungen, die Normen erzeugen, und das heißt: als Kommunikationssystem. ${ }^{226}$ Rechtsprechung ist daher als Rechtserzeugung in Zusammenarbeit mit dem Gesetzgeber und nicht mehr als bloße Rechtserkenntnis aus dem Gesetzestext zu begreifen. Dann stellt sich für den Rechtsstaatsgedanken ein neues Problem: das Normieren des Normierens. ${ }^{227}$ Der Rechtsstaat greift zu kurz, wenn er die Anwendung des Rechts fordert, denn dieses wird erst im Verfahren geschaffen. Weder das Gesetzbuch noch die Methodik kann das Recht vorgeben. Nur im Prozess gewinnt es seine Bestimmtheit.

Wie kann Rechtserzeugung aber legitim sein, wenn sie diese Legitimität nicht aus einer vorgeordneten Quelle schöpfen kann? Die Legitimität findet sich zunächst bei dem vom Gesetzgeber geschaffenen $\mathrm{Ge}$ setzestext. Dieser bildet für die richterliche Entscheidung lediglich ein Präskript. Dieses Präskript wird von den streitenden Parteien unterschiedlich aufgenommen. Damit der Richter seine Entscheidung rechtfertigen kann, hat er die Legitimität des Gesetzestextes zu übertragen. Dabei kann er sich nicht allein auf die sprachliche Bedeutung verlassen, sondern muss die ganze Argumentation im Verfahren berücksichtigen. Die dort vorgelegten Umschriften des Gesetzestextes zu Recht muss er diskutieren und die argumentativ gültige Lesart des Gesetzes seiner Entscheidung zugrunde legen. Erst die Argumentation im Verfahren transportiert die Legitimität vom Gesetzgeber zum Richter. Hier setzt das Konzept der Umschrift an.

dung, in: KritV 1988, 1 ff., prozeduralistisches Rechtsparadigma; vgl. JURGEN HABERMAS, Faktizität und Geltung. Frankfurt am Main 1992, Kapitel IX.

222 AXEL GORLITZ, Mediales Recht als politisches Steuerungskonzept, in: DeRS. (Hrsg.), Politische Steuerung sozialer Systeme. Pfaffenweiler 1989, 13 ff.

${ }^{223}$ GUNTHER TEUBNER, Reflexives Recht, in: ARSP 1982, 13 ff.; DERS., Recht als autopoietisches System. Frankfurt am Main 1989, 81 ff.; GUNTHER TEUBNER/HELMUT WILLKE, Kontext und Autonomie: Gesellschaftliche Selbststeuerung durch reflexives Recht, in: ZfRSoz 1984, 4 ff.; HELMUT WILLKE, Ironie des Staates, Grundlinien einer Staatstheorie polyzentrischer Gesellschaft. Frankfurt am Main 1992.

${ }^{224} \mathrm{Vgl}$. zu einem Überblick von weiteren Begriffen AXEL GÖLITZ/RÜDIGER VOIGT (Hrsg.), Postinterventionistisches Recht. Pfaffenweiler 1990.

225 Vgl. Gralf-Peter CAlliess, Prozedurales Recht. Baden-Baden 1999, 136.

226 Vgl. dazu übereinstimmend für die Diskurstheorie JÜRGEN HABERMAS, Theorie des kommunikativen Handelns. Frankfurt am Main 1981; Ders., Faktizität und Geltung, Frankfurt am Main 1992, für die Systemtheorie NIKLAS LUHMANN, Soziale Systeme. Frankfurt am Main 1984; DeRS., Das Recht der Gesellschaft. Frankfurt am Main 1993, und GUNTHER TEUBNER, Recht als autopoietisches System. Frankfurt am Main 1989.

227 Vgl. dazu CALLIESS, Prozedurales Recht (Fn. 225), 149. 
Bezogen auf die Transkriptivität von Recht siedelt die Frage nach der Legitimität in der Differenz von Präskript und Skript. Das Skript behauptet, das Präskript zu artikulieren, und ruft damit die Postskripte auf, welche diese Behauptung thematisieren. Wenn mit dem Postskript eine Position zum Transkribieren eingenommen wird und dieses befragt wird, kann man von einer parasitären Beziehung sprechen. ${ }^{228}$ Das Postskript nistet sich in die Transkription ein und zehrt von ihr. Sie muss sich nun beweisen, indem sie sich an das mit ihr vorgestellte Präskript rückwendet und sich als dessen Umschreibung legitimiert. Damit stellt sich die Frage der Legitimierung, die zum Teil des fortschreitenden Vorgangs des Transkribierens wird. Das Verhältnis des Parasiten, der Störung und Aufstörung der Transkription, wird also als Kritik nutzbar gemacht.

Wenn man den Parasiten ausrotten will, führt dies dazu, dass die Transkription nicht mehr problematisiert werden kann. Juristen kennen das als die Prätention einer Rechtserkenntnis aus dem Gesetz, die jede sie thematisierende Einrede als unangebracht abweist. Es ist dies der Duktus einer juristischen Semantik, die sich mit dem Normtext allein schon im Besitz seiner Bedeutung glaubt und von daher meint, andere Sinngebungen als Abweichung oder Fehler zurückweisen zu können. Wenn man das Verfahren aus seiner dienenden Rolle befreit, wird deutlich, dass man es für den Übergang vom Präskript des Gesetzestextes zur Entscheidung als dessen legitimer Umschrift benötigt. Durch den Gegensatz der Rechtsmeinungen im Verfahren entstehen zu jeder Umschrift des Gesetzestextes wieder Postskripte, die ihre Berechtigung in Zweifel ziehen. Dies erlaubt eine reflexive Position: als Relation auf die Relation, wie sie in den Verhältnissen von Skript und Postskript impliziert ist. ${ }^{229}$

Will man das im Postskript praktizierte parasitäre Moment anerkennen und aufnehmen, so darf man aber nicht die übliche Bewertung des Parasiten einfach ins Positive wenden und Störung als solche emphatisch affirmieren, begäbe man sich doch sonst jeglicher Korrekturmöglichkeit. Vielmehr kommt es darauf an, Störungen in ihrer spezifischen Ambivalenz, prozessual: ihrer Umschlägigkeit, zu beobachten. ${ }^{230}$ Auf diesem Wege muss das Kunststück gelingen, dem notwendig in Pluralität zerstiebenden Recht auf rechtliches Gehör in einer Weise Rechnung zu tragen, dass zugleich der Gesetzesbindung Genüge getan ist. Das Gesetz löst sich zunächst in die Vielfalt und - mehr noch - den Widerstreit der Lesarten auf. Jede der Parteien stört die andere auf.

228 Dazu StANITZEK, Transkribieren (Fn. 114), 7 ff., 18.

229 Ebd.

230 Ebd. 
Damit erfolgt eine gegenseitige Parasitierung. ${ }^{231}$ Um die Anforderungen an ein legitimes Verfahren zu gewinnen, ist dieses Verhältnis in seiner performativen Logik ${ }^{232} \mathrm{zu}$ beleuchten.

\section{Die Logik des Verfahrens: \\ Störung und Transparenz}

Die neuere Medientheorie schlägt zur Erfassung der performativen Logik von Kommunikation die Kategorien von Störung und Transparenz vor. ${ }^{233}$ Diese stellen zwei polare Zustände der Verständigung dar, die auf zwei entsprechende Modi der Sichtbarkeit verweisen: in der Störung zeigt sich das Medium selbst, während es in der Transparenz das Mediatisierte ist, welches wahrnehmbar wird.234 Entscheidend ist nun, dass sich Verständigung in einem beständigen Wechselspiel von Störung und Transparenz vollzieht. Das Umschreiben des Präskripts stellt Sinn und damit Transparenz her. Mit der Wahrnehmung des Ausdrucks kann die Transparenz durch Problematisierung aufgestört werden. Diese Störung muss dann durch eine erneute Überführung in Transparenz beseitigt werden. Wenn das Postskript dann das entsprechende Skript ausdrücklich macht, indem es die erfolgten Transkriptionen zur Disposition stellt, kann man auch sagen, dass hier in einem beständigen Übergang von Transparenz in Störung und von Störung in Transparenz „Implizites explizit gemacht wird und umgekehrt". ${ }^{235}$

Das Präskript ist kein Ausdruck einer vorgeordneten Wesensebene. Es repräsentiert nicht eine zugrunde liegende Bedeutung, es präsentiert Argumente. Darin zeigt sich abermals die im Konzept von Performanz und Transkriptivität vollzogene Abkehr vom Paradigma des Referentiellen und Repräsentativen.236 Für das Wechselspiel von Störung und Transparenz ist es jedenfalls erst einmal offen, wohin es führt. Es hebt den Vorhang für "eine semantische Aushandlungsbühne für die sprachliche Sinnkonstitution sowohl für die metaleptische Konstruktion der

231 LUDWIG JÄGER, Störung und Transparenz. Skizze zur performativen Logik des Medialen, in: SYBILLE KRÄMER (Hrsg.), Performativität und Medialität. München 2004, 35 ff., 38 ff., im Anschluss an den Begriff der "strukturellen Parasitierung" bei DERRIDA, Signatur Ereignis Kontext (Fn. 65), 15 ff., 21.

232 Siehe dazu KRÄMER, Was haben ,Performativität' und ,Medialität' miteinander zu tun? (Fn. 110), 13 ff., 14.

233 Siehe JÄGER, Störung und Transparenz (Fn. 231), 37 ff., vor allem $41 \mathrm{ff}$.

${ }^{234} \mathrm{Vgl}$. KRÄMER, Was haben ,Performativität' und ,Medialität' miteinander zu tun? (Fn. 110), 13 ff., 25

$235 \mathrm{Ebd}$.

236 Ausführlich dazu JÄGER, Transkriptivität (Fn. 114), 19 ff., 19 ff. 
eigenen Redeintention, als auch für die interaktive Verständnissicherung im performativen Vollzug der Redeentfaltung. Sprechen und Schreiben verfahren also insofern transkriptiv, als bei beiden Arten symbolischer Performanz in den produktiven Prozess der Zeichenhervorbringung konstitutiv rezeptive Momente der Selbstlektüre eingebaut sind, die sich als Formen der Selbsttranskription beschreiben lassen. ${ }^{237}$

Daraus ergibt sich, dass Störungen und ihre transkriptive Bearbeitung ein zentrales Verfahren der sprachlichen Sinnproduktion darstellen: Störung wird so zu einem "Produktivitäts-Prinzip sprachlicher Sinngenese". ${ }^{238}$ Wenn Störungen eintreten und in Transparenz überführt werden, kann dies gerade nicht als Korrektur von Fehlern begriffen werden. Das Postskript parasitiert die Beziehung zwischen Präskript und Transkript. Aber wir können den Parasiten nicht ausmerzen, weil wir ohne ihn gar nicht kommunizieren könnten. Deswegen stellt sich die Frage, wie man sich mit ihm arrangieren kann. Der Parasit unterbricht durch Störung die Sinnproduktion. ${ }^{239}$ Diese Unterbrechung durch Störung ist ambivalent, weil sie sowohl zur Zerstörung als auch zur Entwicklung des Systems beitragen kann. Wenn sich ein System an einen Parasiten gewöhnt, verwandelt sich der störende Lärm in sinnhaftes Rauschen. ${ }^{240}$ Allerdings produziert der Parasit keine Bedeutung, sondern zwei entgegengesetzte Bedeutungen. ${ }^{241} \mathrm{Er}$ ist gerade die Differenz der Entgegensetzung als thermischer Erreger des Konflikts. ${ }^{242}$ Das Parasitäre im Postskript ist daher nicht Bremse, sondern Motor des Prozesses. Es signalisiert keinen Defekt der Äußerung und keine performative Aberration von einer präverbalen Redeintention. Vielmehr fungiert es als Fingerzeig für die Notwendigkeit der transkriptiven Weiterbearbeitung der Äußerung. 243

Der Konflikt gegenläufiger Lesarten ist geradezu das Movens des Verfahrens der Rechtserzeugung aus dem Normtext. Die auf den Normtext bezogenen Argumente der Parteien entfalten ein kompliziertes Spiel der Differenz- und Identitätsbildung. Man kann dies als Vorgang "der Irritation und Wiedereinpendelung des parasemischen Gleichgewichts" begreifen. ${ }^{244}$ Unter der Notwendigkeit von Entschei-

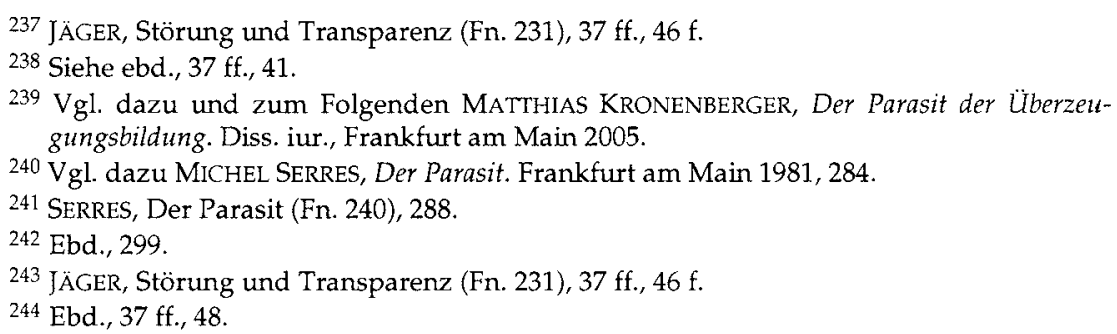


dung gewinnt dies seinen besonderen Charakter, der dann auch auf den Vollzug von Legitimierung verweist. Das Besondere besteht darin, dass die divergierenden Lesarten der Parteien jeweils nur einseitig als "Störung" gesehen werden. Entsprechend sind die Postskripte in einem zwischen ihnen oszillierenden Prozess abzuarbeiten, indem sie entweder durch ihren Bestand in Transparenz gewendet oder aber durch Abstoßung ungeschehen gemacht werden. Die beiden in der Argumentation vor Gericht einschlägigen Praktiken für diese "Aufzehrung" von Postskripten als Störung entweder durch Absorbierung oder Elimination sind die Integration und die Widerlegung von Argumenten. Mit ihnen wird Geltung hergestellt als eine Einkehr der Umschreibung von Normtext zu Recht zu sich selbst zurück, die aufgrund ihres argumentativen Erfolges als legitim beansprucht werden kann.

Die Logik des Verfahrens fordert, den Text von Recht durch den Widerstreit der Lesarten als dessen Transkription in Arbeit zu nehmen. Praktisch lässt sich der Gang dieser Arbeit am Text von Recht als semantischer Aushandlungsprozess entlang der Grundzüge der Argumentationssituation beschreiben. Die von den Parteien vorgetragenen Umschreibungen des Normtextes in Recht schließen sich gegenseitig aus. Dies macht den Streit aus. Die Stellungnahme einer Partei ist jeweils Postskript zur Stellungnahme der anderen. Keine der beiden Lesarten ist damit evident. Denn ihre Transparenz wird von der gegnerischen Lesart gestört. Beide machen aber mit ihren widerstreitenden Lesarten deutlich, dass es um denselben Gesetzestext als Präskript geht. Wenn das Gericht entscheiden will, muss es den argumentativen Streit der Parteien nutzen. Am Ende des Verfahrens kann dann eine Lesart evident sein. Aber diese Evidenz ist keine, die an das Bewusstsein der beteiligten Personen gebunden ist, sondern es ist eine Evidenz, die im Verfahren erst erzeugt wurde. Wenn alle gegnerischen Argumente integriert oder widerlegt sind, wird die verbleibende Lesart evident. Integrieren erfolgt, wenn Störung durch gelungene Absorbierung in Transparenz umgemünzt wird. Widerlegen gelingt, wenn das gegnerische Postskript durch Verdrängung getilgt wird, indem der von ihm behauptete Bezug zum Gesetzestext zerrissen wird. Alle Störung ist dann beseitigt und es bleibt nur noch die geltende Lesart in vollkommener Transparenz.

Juristisches Argumentieren ist das Abarbeiten von Argumenten im Rechtsstreit zugunsten der Durchsetzung der eigenen Lesart. Dieses Interesse kennzeichnet das Vorgehen der Parteien: Es geht ihnen darum, alle denkbaren Gründe für die eigene Position einzunehmen und sie im gleichen Zuge der gegnerischen Position zu versagen. Dafür kann im Verfahren in Gestalt der Lesarten immer nur Text für Text 
einstehen und die Frage der Legitimität ist allein die praktische einer Entscheidung über deren Bestand. Wenn ihre Lesart des Normtextes evident werden soll, müssen die Parteien die Störung durch Gegenargumente abarbeiten und Transparenz erzeugen. Dafür können sie sich nur solcher Texte bedienen, die aktuell nicht in Zweifel stehen und die so für eine Bedeutung von Recht genommen werden können. Juristisches Argumentieren erweist sich damit als das Bemühen um eine Anreicherung des anerkannten Texts von Rechts durch den eigenen. Es zielt darauf ab, sich in das alleinige Recht auf eine Entscheidung zu setzen. Dennoch hat es sich in diesem Anspruch stets zu legitimieren. Einer Akzeptanz durch alle Beteiligten steht dabei der sich praktisch durchsetzende Charakter eines Widerstreits entgegen. Es bleibt nur die Entscheidung zugunsten einer Position, die sie in ihrer Begründetheit zu Ungunsten der anderen ratifiziert. An die Stelle der Akzeptanz muss man deswegen die Geltung von Argumenten setzen. Auch für diese Geltung muss aber der Prozess des Argumentierens selbst sorgen.

Wenn man also die Steuerungskraft des Gesetzes und die Rolle der Gerichte realistisch einschätzt, muss sich die Rechtstheorie von der zu einfachen Vorstellung bloßer Rechtsanwendung lösen, das legalistische Rechtsverständnis verabschieden ${ }^{245}$ und als medienreflexives Rechtsverständnis reformulieren. ${ }^{246}$ Die Sprache als solche ist überfordert, wenn man ihr aufbürdet, aus dem tatsächlichen Prozess der Erzeugung von Recht eine bloße Erkenntnis von Bedeutungsgegenständen zu machen. Sprache funktioniert allein als Vorgang der Verständigung. Daher kann man Normativität nicht aus ihr beziehen; man kann sie nur in der Sprache herstellen. Nicht die Sprache gibt dem Urteil Halt, sondern die Argumentation.

Das Recht liegt nicht in einer Struktur hinter der Welt. Es kann sich nur in den Wechselfällen des Verfahrens herausbilden. Das Verfahren ist aber nicht die Aufführung eines im Gesetz schon vorgeschriebenen Stücks. Es ist eher Improvisationstheater. Das Recht ist nicht seine Quelle, sondern seine Leistung. Diese kann es nur erbringen, wenn der Richter den Streit der Parteien für seine Begründung arbeiten lässt. Darin liegt die Kunst, Recht geschehen zu lassen.

\footnotetext{
245 Programmatisch für eine nachpositivistische Rechtstheorie formuliert bei ALEXANDER SOMEK/NIKOLAUS FORGÓ, Nachpositivistisches Rechtsdenken. Wien 1996, 357ff.

${ }^{246}$ Vgl. dazu auch ALEXANDER SOMEK, Rechtssystem und Republik. Wien 1992, $475 \mathrm{ff}$.
} 